\title{
Behavior of Mock-Ups in the California Technical Bulletin 133 Test Protocol: Fabric and Barrier Effects
}

T. J. Ohlemiller and J. R. Shields

May 1995

Building and Fire Research Laboratory

National Institute of Standards and Technology

Gaithersburg, MD 20899

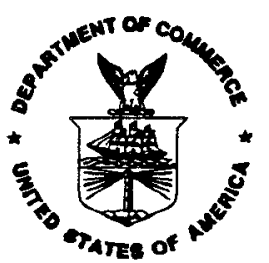

U.S. Department of Commerce

Ronald H. Brown, Secretary

Technology Administration

Mary L. Good, Under Secretary for Technology

National Institute of Standards and Technology

Arati Prabhakar, Director 


\section{TABLE OF CONTENTS}

Abstract

Introduction

Experimental Details

Mock-Up Geometry

Full-Scale Test Details

Camera Observations

Heat Flux Measurements

Cone Calorimeter Tests

Upholstery Materials

Results and Discussion

Cone Calorimeter Heat Release Rate

Cone Tests with No Fabric

Effect of Varying the Cone Heat Flux

Heat Feedback Flux in the Cone Calorimeter

Full-Scale Rate of Heat Release

Heat Release During Burner Exposure

Fire Growth Pattern During Burner Exposure

Heat Fluxes During Burner Exposure

Correlation of First Peak with Cone Calorimeter

Dimensional Analysis of Fire Growth on a Chair

Effect of Mock-Up Geometry

Heat Release After Burner Exposure

Barrier Failure Mechanism

Correlation With Cone Calorimeter 


\title{
Behavior of Mock-Ups in the California Technical Bulletin 133 \\ Test Protocol: Fabric and Barrier Effects
}

\author{
T. J. Ohlemiller and J. R. Shields
}

\begin{abstract}
Twenty-seven material combinations (seven fabrics, four barriers and two polyurethane foams) were tested in four cushion mock-up form in accord with California Technical Bulletin 133 using a furniture calorimeter. These same material combinations were also tested in triplicate, mainly at $35 \mathrm{~kW} / \mathrm{m}^{2}$, in the Cone Calorimeter. Both mock-up and Cone sample behavior were recorded on video to facilitate behavioral comparisons of the samples; distinct differences were noted for thermoplastic fabrics. Heat fluxes were also measured on both scales; the effective "external" flux in full-scale can exceed $50 \mathrm{~kW} / \mathrm{m}^{2}$. The mock-up behavior always comprised at least a heat release peak during the $\mathbf{8 0}$ second gas burner exposure; it often included a later and larger peak as well. The first peak could not be adequately predicted by averaged heat release data from the Cone. A dimensional analysis suggested a possible dependence on four additional parameters, three of which can be obtained from the Cone. A statistical fit of the available data to these more complex types of correlations appears to work best for charring fabrics; it helps improve the correlation for all types of fabrics but two material combinations were outliers. The second heat release peak in the mock-up tests is attributable to a "basal melt fire" mechanism; efforts to correlate this behavior with Cone results have been limited thus far and show little success.
\end{abstract}

Keywords: Cone Calorimeter, fabrics, fire barriers, furniture, furniture calorimeter, heat release rate

\section{1) Introduction}

Upholstered furniture used in public occupancies (hotels, hospitals, airport lounges, etc.) is recognized as having a potential for involvement in multi-fatality fires. It thus is coming under increasing scrutiny as a possible major contributor of heat, smoke and toxic gases in such fires.

The California Bureau of Home Furnishings and Thermal Insulation has published a fire test protocol for such furniture [1], refined in conjunction with NIST [2,3,4]. The test is designated as California Technical Bulletin 133 (CB 133). This test essentially looks at the behavior of an 
item of furniture subsequent to ignition, placing strict limits on the allowed levels of heat release rate, total heat evolved, smoke obscuration and $\mathrm{CO}$ levels in a defined room enclosure. This protocol is mandated in four states (California, Illinois, Minnesota and Ohio) and is under consideration in the legislatures of several other states.

The trend toward broader adoption of CB 133 has prompted significant efforts on the part of the suppliers to this market segment to provide materials and end products which assure customers of compliance with its requirements. Some of the larger manufacturers have made the necessary effort to assure that their entire product line is in compliance $[5,6]$. This typically requires an enormous investment in empirical (CB 133) testing.

There is a distinct need for a less expensive test procedure that has been shown to relate quantitatively to performance in CB 133. If this is not available, there is at least a need for some quantitative guidelines in the form of a database that smaller manufacturers can use to help locate minimum heat release combinations among the enormous number of permutations that are possible in furniture design.

The present study is an attempt to provide information that addresses both of the above needs. The study is focussed on rate of heat release as the measure of greatest interest and relevance to the hazard that a fire poses. The small-scale test protocol whose results we hope to correlate with full-scale behavior is that of ASTM 1354, the Cone Calorimeter, since this has shown some predictive ability in the past for furniture behavior [7]. This test is typically run at a fixed external incident flux of $35 \mathrm{~kW} / \mathrm{m}^{2}$; this was done here also. However, in order to gain some insight into the effective "external" fluxes experienced by area elements on the internal seating surfaces of a chair, two heat flux gages were placed on the seat cushion during the tests done here.

Upholstered furniture variables include the fabric composition, the nature of any interliner or barrier layer designed to protect the foam, polyurethane foam composition, external geometry and internal construction details (frame, cushion supports, etc.). In this study the CB 133 steel mockup frame is used as a means to eliminate and constrain construction variables to a single case. In addition, a single external geometry is used; cushions of a fixed size are used in a mock-up of a fully upholstered chair. Only fabric, interliner and foam have been varied with this fixed geometry. In a planned follow-on study, the dimensions of the chair cushions will be varied in a systematic manner.

The use of barrier layers has proved to be a key element in obtaining heat release performance in keeping with the low allowed limit in the CB 133 protocol. These are composed of woven or knitted glass or strongly-charring organic fibers formed into sheets which can be used to totally enclose the polyurethane foam cushioning. It has been noted previously that these barriers sometimes fail to prevent involvement of the foam. One of the goals of this study was to ascertain the reasons behind such failures. 
The range of available upholstery fabrics is vast; fiber blends, in particular, are almost infinitely variable. In choosing a small subset of fabrics for study here, some effort was made to cover the spectrum of fire response but a particular emphasis has been piaced ren the worst end of highly energetic fibers with the thought that it is useful, in a very limited determine what is required to deal with some of the most challenging cas

\section{2) Experimental Details}

Mock-Up Geometry. Figure 1 shows a sketch of the mock-up geometry used throughout this test series. This was varied in only one test when the arm cushions were omitted. The inclusion of full thickness arm cushions makes this geometry rather severe in the sense that the peak rate of heat release tends to be enhanced by the radiative interactions of the four internal seat surfaces. Given this, plus the emphasis noted above on rather energetic materials, it is not especially surprising that many of the peak heat release rates seen in this study exceeded the allowed maximum of $80 \mathrm{~kW}$ in the CB 133 protocol.

The cushions are held in the configuration of Figure 1 by the CB 133 steel mock-up frame. This frame normally makes minimal contact with the outer surfaces of the cushions and, in fact, the cushions can fall through the frame if they shrink sufficiently during a test. Since a fallen cushion can lead to a severe fire if its flames play on the bottom of other cushions and since this seems somewhat of an artifact of the use of the frame rather than a complete chair, the mock-up frame was modified early in the test series by the addition of short, stiff steel wires centered beneath the front and back ends of both arm cushions. These prevented the arm cushions from falling down through the mock-up frame while, at the same time, showing little or no effect on the spread of flames over the cushion surfaces.

Full-Scale Test Details. Ignition was achieved by means of the standard, square-ring, propane gas burner, positioned on the inner surfaces of the seat as specified in the test protocol. The propane flow rate, which was calibrated before each test, varied from 12.9 to 13.15 NTP liters/min.

Since rate of heat release was the primary variable of interest, the tests were performed in the NIST furniture calorimeter rather than in a standard room enclosure. This device measures rate of heat release by means of oxygen consumption calorimetry. It was calibrated at frequent intervals (daily in the second half of the test series) using a natural gas burner, typically at about $75 \mathrm{~kW}$.

The oxygen meter used in the analysis of the exhaust showed a slight tendency to drift during testing. Thus the post-test reading for room air (after passage through a cold trap) might have been anywhere from 20.88 to $20.95 \%$. To compensate for this during the computation of heat release rate as a function of time, a baseline correction was used. The baseline was taken to be linear and was fitted through the initial $(20.95 \%)$ reading and the final reading obtained several minutes after the fire was completely out. This had very little impact on the rate of heat release 
during the gas burner exposure but a more significant effect on the readings near the end of the test. It should have no influence on any inferences made from the data reported here.

Camera Observations. As noted above, one goal was to ascertain the manner in which interliners sometimes fail to protect the foam inside the cushions. Tow ard this end, extensive video and photo documentation of the mock-up behavior was included. Video cameras were used to follow the course of the burn process both from the front (continuously) and from the back (intermittently). In the second half of the test series, a large mirror was used to observe events on the bottom surfaces of the mock-up cushions; these were recorded verbally on the video tapes. A $35 \mathrm{~mm}$ camera viewing the front of the mock-up was used to obtain higher resolution records of the fire progress at fixed intervals (30 or 45 seconds). An infrared imaging camera with a 10.6 micrometer flame filter was used to view the front of the mock-up both to look for any indications of fire growth internal to the cushions and to provide data on cushion surface temperatures.

Heat Flux Measurements. Figure 2 indicates the typical placement of the two heat flux gages used on the seat cushion surface. These were Medtherm ${ }^{1}$ Schmidt-Boelter gages which measure the total flux incident on the gage surface. Ideally these gages would be embedded within the cushion so that the sensing surface was flush with the cushion surface. This was impractical here since it would have ruptured the interliner on the cushion. The gage is only $12.7 \mathrm{~mm}$ thick so the sensing surface was elevated this amount above the cushion surface. For the buoyant and turbulent upward flow on the seat cushion, this displacement should yield much less of an error in reading the convective portion of the heat transfer to the cushion surface than would be the case on a vertical cushion surface (where the sensing surface could be well out of the boundary layer).

It should be noted that this type of flux gage is essentially a differential thermopile whose cold junctions need active cooling. Furthermore, the sensing surface is only a few degrees Celsius above the cold junction temperature. To prevent condensation of water on the sensing surface, each gage was cooled with heated silicone oil pumped from a controlled-temperature reservoir. Each gage had a separate thermocouple inside to monitor the cold junction temperature.

In some of the early tests the heat flux gages were placed side by side, in theory seeing the same flux, and cooled at widely differing temperatures (ca. 100 and $190^{\circ} \mathrm{C}$ ). The goal was to extrapolate the temperature dependence of the flux data to the measured surface temperature to correct for the overestimate of the convective heat flux component inherent in a sensor surface being cooler than the cushion surface. The noise in the data quite effectively precluded this. There was no consistent difference in the fluxes seen by the two gages. This implies that the convective component is substantially smaller than the radiative component in these tests.

\footnotetext{
${ }^{1}$ Certain product names are mentioned for clarity and completeness only; this does not imply any endorsement by NIST nor does it imply that they are necessarily the best product for the noted purpose.
} 
Throughout most of the tests the gages were run at temperatures in the $120-150^{\circ} \mathrm{C}$ range. They were also calibrated in this temperature range. The gage temperature could vary as much as 20 ${ }^{\circ} \mathrm{C}$ during a test as the fire intensified around it and its coolant lead lines. From the weak sensitivity of the calibration to coolant temperature, this appears to have had an insignificant effect on the accuracy of the flux values observed.

Thermoplastic fabrics threatened to falsify the flux data by curling over the gage surface as the fabric melted and contracted during fire growth. In the second half of the test series this was precluded by the use of two or three needles placed around the gage to block the curling fabric. This proved to be quite effective.

The gages were fixed in space by their lead lines. If the seat cushion continued to burn during a test, it typically shrank significantly causing its surface to pull away from the flux gages. The flux data reported here are thus only those obtained during the CB 133 burner exposure; cushion shrinkage was not a problem during this 80 second interval.

Cone Calorimeter Tests. Sample preparation for Cone testing followed the new protocol developed for the on-going European Community furniture flammability study [8]. This technique, though tedious, is reported to significantly improve inter-laboratory reproducibility. It imposes very tight tolerances on all dimensions (fabric and foam). It also calls for pre-forming the fabric over a wooden block so that it can be precisely glued into a shape that will enclose the foam block on the top and sides.

The samples were tested in a horizontal orientation typically only at an incident flux of 35 $\mathrm{kW} / \mathrm{m}^{2}$. Certain fabric/interliner/foam combinations were tested at other fluxes, as well, as reported below.

If a sample showed any tendency to extinguish after the initial peak of heat release, the spark igniter was re-inserted above the sample surface and activated there until re-ignition occurred or ten minutes had elapsed since the extinction occurred. Generally this caused no ambiguity in the results, i.e., when the igniter caused re-ignition and the appearance of a second heat release peak, that peak occurred after a clear interval of essentially zero heat release. When such wellseparated second heat release rate peaks appeared, they were the product of re-ignition by the spark or, once or twice, by the Cone heater itself. Thus, if one wanted to know what the material performance in the Cone would have been if the igniter had not been on, one need only ignore the separated second peak.

Heat flux measurements were also made with a limited number of samples in the Cone for comparison with the full-scale flux measurements. The same type of flux gage was used, positioned near the center of the top sample surface. The gage was cooled with hot water at 85$95{ }^{\circ} \mathrm{C}$ to prevent water condensation on its surface. As the sample surface shrank downward during burning, the gage was lowered manually to stay in contact with the top surface. Typically a roughly pyramidal "flame tent" $10-15 \mathrm{~cm}$ high formed early in the test as the fabric became fully involved. The data during such a time were the most meaningful and are used below in the 
comparisons with the full-scale results. As the fabric began to burn out (causing the typical dip in heat release rate that forms the downside of the first heat release peak), the flame tent periphery would fluctuate increasingly, eventually passing over the gage surface. This direct flame front contact with the sensor surface would cause momentary upward spikes in the flux reading.

Upholstery Materials. Table 1 gives a brief description of the materials used in this test series. The barrier materials were bonded to the back of the fabrics before the cushions were manufactured at a commercial upholstery plant. (Extra pieces of the same bonded fabric/barrier combinations were tested in the Cone Calorimeter.) All cushions had welt cords and were sewn with an aramid thread. Because nylon based zippers were inadvertently used and these were found to fail in early tests, the zipper opening in all cushions used subsequent to the first two tests was sewn tightly shut with aramid thread. This proved fully effective in stopping any opening of the barrier in this region. (This region was always placed so that it could be observed during the mock-up tests, i.e., the zippers faced up or to the front.)

Table 2 lists the twenty-seven material combinations tested in both the Cone Calorimeter and in full-scale mock-ups. Note that Barrier BB was used only in Sets 23 and 26. Also note that Set 26 , unlike all of the other sets, has substantial amounts of flame retardants in all of its elements (fabric, barrier and foam).

Cone tests were run in triplicate. Most of the full-scale tests were run as single replicates but duplicates were available for six of the material combinations. In some cases these duplicates were used for other purposes (e.g., to repeat the first test in which zipper failure gave misleading results or to run a two cushion test to compare with a four cushion test.)

\section{3) Results and Discussion}

Cone Calorimeter Heat Release Rate. Figures 3 to 11 show the heat release rate curves obtained for the twenty-seven sets of materials. They are arranged in groups of three with a common fabric and foam but differing barriers. All of the tests in this group of figures were run at an incident radiant flux of $35 \mathrm{~kW} / \mathrm{m}^{2}$.

At this flux the typical heat release curve consisted of two major peaks. The first is believed to be dominated by the fabric with some potential contribution from the barrier (if organic) and perhaps a contribution from the foam (see below). The second peak was dominated by the foam but usually included combustion of the substantial quantity of fabric on the sides of the sample, as well. $^{2}$ The mass of this side fabric was more than twice that on the top of the sample but

\footnotetext{
${ }^{2}$ Recall that the spark igniter was used essentially continuously and thus sometimes caused reignition of a sample after the first peak was ended. These cases are made evident by the clear separation between peaks with a period of essentially zero (sometimes $5-10 \mathrm{~kW} / \mathrm{m}^{2}$ ) heat release before the second peak.
} 
its combustion was slowed by its being sandwiched between the barrier and the side of the aluminum foil wrapper that encompassed the sample. From a limited number of deliberately interrupted tests, it appeared that by the time the foam was actively burning in the second peak, it had been reduced to a thin layer of liquid in the bottom of the foil wrapper.

The pre-ignition and burning behavior of the fabric on top of the sample was highly variable with fiber type. The simplest behavior was seen with the fabric based on $100 \%$ cotton. This fabric simply charred in place, though later that char was oxidized in an erratic manner with much of it ultimately disappearing. Most of the fibers in Table 1 are thermoplastic and thus tend to liquify when heated. However, the behavior of the fabric seemed to depend on some poorly understood competition between weave-induced stresses, heat-induced stresses and relaxation of both types of stresses when the fibers began to melt.

Most of the thermoplastic fabrics split open extensively before ignition, exposing roughly $75 \%$ of the barrier on the top surface of the sample. The polyester, the nylon and the lighter weight polypropylene behaved in this manner. In contrast, the heavier polypropylene hardly split open at all, exposing only $0-10 \%$ of the barrier on the top sample surface. The polyester/cotton blend behaved as though it was dominated by the cotton, showing minimal splitting (which tended to be uni-directional). Clearly the behavior of a fiber blend will depend on the relative charring tendencies of the fibers and their pattern of distribution in the weave.

In spite of the tendency of several of these fabrics to split open and expose a large area fraction of barrier material, the small scale of the Cone sample assured that the fabric flames could close into a single "tent" covering the entire sample top. This tends to yield a rate of heat release that is some average of the fabric covered portion of the sample top and the uncovered (exposed barrier) portion. In the full-scale tests to which these Cone tests are to be related, the scale of the splitting of the fabric was larger, precluding flame closure over the exposed barrier regions. This is a possible basis for differences in rate of heat release behavior between the two scales. This may be a critical issue, as will be seen below.

Note that for some sets, there was no second heat release peak. This only occurred with Barrier $\mathrm{C}$ (woven glass). Evidently, this barrier, under the specific conditions of fabric, foam and flux used, substantially reduces what is probably a predominantly radiative heat flux reaching the top of the melted foam. After the first peak ends, this reduced flux is unable to generate an ignitable mixture above the sample (the spark igniter continued to be present).

Even when there was a second peak, there often was an interval of no flaming between peaks. This was seen with all of the barriers, but was most common with Barrier $C$ and least often with Barrier A (non-woven aramid). Presumably the cause is the same as above but the reduced heat flux to the foam is more marginal; eventually the foam melt boils and generates an ignitable mixture, giving rise to the second heat release peak.

Table 3 lists the 60 second and 180 second average values of the Cone data for the various material sets in this study. The 180 second value has been used in the past for correlation with 
full-scale results [7]. As will be seen below, the 60 second average is more logical for some aspects of the CB 133 test. Inspection of Table 3 shows that Barrier C (woven glass) consistently gives lower average heat release values than the other two barriers for the same fabric/foam combination. The differences can be quite large for the 180 second averaged data; this reflects the lack of a second peak or a non-flaming interlude between peaks with Barrier $C$. The differences are less dramatic but still substantial for the 60 second average data that emphasize the fabric behavior.

This significant influence of the barrier in a domain thought to be dominated by the fabric prompted a further look. Initially it seemed plausible that the differences among barriers for a given fabric resided in the physical behavior of the fabric atop the barriers. Suppose that, during a Cone test, a thermoplastic fabric tends to melt and bead up into a smaller area when its on top of one type of barrier but that it tends to retain more of its initial $100 \mathrm{~cm}^{2}$ top surface area atop another barrier (due to adhesion and/or wetting differences). The fabric melt in the beaded-up case should tend to burn more slowly (and longer) than the latter stretched-out case because it has less surface area exposed to flame and Cone heat fluxes. Video tapes of the tests failed to provide obvious support for this idea; the extent of splitting open of the fabric appeared to be primarily a function of the fabric, not the barrier. Similarly, no unambiguous support could be found for the apparent tendency of some of the fabric melts to pass through (by wetting and dripping through) some of the barriers and thus be relatively protected from flame and Cone heat fluxes. There could still be something to these potential sources of barrier effects. For example, the rate of heat release from the fabric should be directly proportional to the area fraction which its melt occupies on the top of the sample. More sensitive and quantitative techniques would be needed to assess whether this is the source of the barrier dependence.

Cone Tests With No Fabric. Cone tests were run with the barrier alone atop Cal 117 foam. Figure 12 summarizes the results. Barrier $C$ (woven fiberglass) gave the least heat release; Barrier B (knit) gave the most. In the case of Barrier $C$, placing it atop inert Kaowool instead of foam yielded essentially no heat release, implying that the peak in Figure 12 is due to foam burning. Barrier A (aramid) atop Kaowool gave a peak only about ten percent of the height shown in Figure 12, again suggesting that foam burning contributed most of the peak in Figure 12.

Barrier B alone atop the foam permitted two substantial peaks of heat release with no extinguishment interval in between. The second peak occurred because of a phenomenon noted in some full-scale tests with this barrier also: flames appeared inside the barrier, even though it stayed intact and developed no new holes. This barrier, when charred, has the largest inherent holes of the four barrier versions examined. Whether flames propagate through these holes or ignite internally due to a high barrier temperature is not clear. However, these flames do tend to compromise the purpose of the barrier. Note in Table 3, however, that Barrier B gave ,60 second Cone heat release performance equivalent to Barrier $C$ with the light polypropylene fabric and similar performance equivalent to Barrier $A$ with cotton and modacrylic-nylon. These last two fabrics are char formers which tend to protect both barrier and foam beneath them. Evidently, 
barrier performance is coupled to the fabric it lies under and one cannot generalize about the relative performance of Barriers $A$ and $B$.

A point to note with regard to the results in Table 3 and the relation between them and full-scale behavior (discussed below) is that they are the consequence of complex heat transfer pathways. It is the overall response to these heat transfer processes which determines the net burning rate of the sample and thus its heat release rate. During the first peak of heat release, radiant heat from the Cone falls uniformly on the top surface of the sample. The top surface itself does not remain uniform very long however, particularly in the case of a thermoplastic fabric. Instead, before ignition, it splits open in an erratic manner resulting in a top partially covered by rapidly melting fabric and partially covered by the exposed barrier. The ability of the radiant heat to affect the foam below the barrier is thus dependent on this splitting pattern. So also is the burning of the fabric melt on the top surface. As the fabric catches flame it quickly builds a flame "tent", described above, which is a non-uniform source of conductive, convective and radiant heat to the top surface of the sample. Penetration of this combined flux to the fabric melt and to the foam below the barrier is also affected by the fabric splitting pattern. The behavior of the fabric may not be the same in the full-scale case, as discussed below.

It is apparent from the above discussion that a firm grasp on all of the factors that control the size of the first peak in the Cone tests is not at hand. Since the fabric is most exposed to both the Cone and flame heat fluxes, it is still expected to be the major contributor to the first peak in most cases. However, fabric splitting to expose the barrier and the properties of that barrier allow a variable contribution from the polyurethane foam.

Effect of Varying the Cone Heat Flux. For one barrier/foam combination under three fabrics, the external radiant flux from the Cone heater was varied. The barrier in all cases was $A$ (aramid) and the foam was Cal 117. The fabrics chosen represented high (Hvy PP), low (MA-N) and intermediate (Cot) rates of heat release at $35 \mathrm{~kW} / \mathrm{m}^{2}$. Figure 13 shows the one minute average rate of heat release as a function of incident radiant flux. Note that the barrier alone, atop the foam is also included. This particular form of the aramid barrier had some additional adhesive content that probably contributed to the heat release seen. The scatter in the data is probably attributable to the fact that the Figure includes data from tests both with and without a flux gage atop the sample. The gage cked roughly ten percent of the top and thus tended to lower the heat release (though this wa not always the case). The lines through the data are second order regression curves.

Figure 14 shows examples of the complete rate of heat release curves as a function of incident flux. Figure 15 shows how the extent of sample consumption varies with this flux. Comparison of the results in Figures 15 and 13 reveals that the drastic reduction in sample consumption at low fluxes is not necessarily accompanied by a sharp reduction in the one minute average heat release rate. Figure 14 implies that this is because the fabric on top of the sample is still consumed with substantial vigor but the foam below it and the fabric on the sample sides are not. Since the fabric dominates the early heat release, failure of the foam and side fabric to burn subsequently has minimal effect in Figure 13, which is based on the first 60 seconds of heat 
release. The failure of the foam to burn at low incident radiant fluxes is presumably a consequence of inadequate transmission of heat through the barrier.

The preceding Cone Calorimeter results will be re-examined when the full-scale results are discussed.

It is worth noting that Figure 13 only adds to the uncertainties discussed above regarding the effects of differing barriers on early heat release rate in the Cone tests. Table 3 shows that all of the polypropylene sets gave substantially higher average heat release rates with Barrier $\mathrm{A}$ than with the other two barriers. Figure 13 suggests that any increased exposure of the barrier/foam with this barrier would not add nearly enough to the overall heat release to explain the observed differences. The reason why this fabric burns more vigorously atop this barrier is not apparent from the available evidence.

Heat Feedback Flux in the Cone Calorimeter. As noted above, many of the tests reported in Figure 13 were conducted with a heat flux gage placed atop the center of the sample. This measures the total flux to the gage surface (ca. $90^{\circ} \mathrm{C}$ ) from the flame and from the Cone heater. The latter is known from separate measurements. Assuming that the flames are essentially transparent to the Cone radiation (a plausible but unverified assumption), one can subtract this flux from the total flux to infer the flux the gage surface receives from the sample flame. If one further knew the surface temperature of the sample, one could correct this gage flux for the difference between the gage and surface temperatures to get the actual flame flux to the surface [9]. Here the surface temperature is poorly defined because the surface material varies from point to point for the split-open thermoplastic fabrics. Thus only the flame heat flux to the gage surface is calculated; it will be compared to the same heat flux from the full-scale tests below.

Figure 16 shows these heat fluxes (denoted as "apparent flame flux") as a function of Cone radiant flux for the three material combinations reported in Figure 13. The error bars reflect the noise level of the data during the time interval when the flame "tent" was intact during the first heat release rate peak. Thus these data primarily are a result of the fabric burning process with some contribution from barrier and/or foam materials.

There are two points to note. First, all three fabrics yielded flame fluxes which did not change significantly with the level of external radiant flux. This was despite the fact that the sample burning rate was changing appreciably, as Figure 13 shows. The increase in heat release rate there was driven entirely by the increase in external flux. One might expect the flame flux to decrease with external flux due to blowing effects. In this horizontal configuration, the flame was apparently far enough away that this blowing effect was negligible. This might not be the case for a vertically-oriented sample surface.

The other point to note is that the different fabrics yielded differing flame flux levels but their relative magnitudes were not necessarily the same as the order of the heat release rates in Figure 13. The polypropylene fabric was highest in both the flame flux to the gage (ca. $25-30 \mathrm{~kW} / \mathrm{m}^{2}$ ) and heat release rate. However, the cotton fabric, which yielded an intermediate heat release rate 
had the lowest flame flux to the gage (ca. $15-20 \mathrm{~kW} / \mathrm{m}^{2}$ ). Despite its relatively higher (ca. 22-27 $\mathrm{kW} / \mathrm{m}^{2}$ ) flame flux, the modacrylic-nylon blend yielded a lesser heat release rate than the cotton. This blended fabric contains a fairly energetic component (nylon) and a strong char former (modacrylic). The combination was woven in such a way that it formed a strong protective shell over the barrier and foam, even as it burned. The high modacrylic content in the fabric itself apparently also made it resistant to the higher flame flux that may have originated with the nylon.

Full-Scale Rate of Heat Release. Figures 17 to 25 show the heat release curves obtained from the full-scale mock-ups; these are arranged in a manner analogous to the Cone Calorimeter data. Note that the ordinate scale varies from one figure to the next. There are one or more heat release peaks in all cases; Table 4 gives the values of the first and of the largest of the later peaks. The first peak occurs typically near the end of the burner exposure and includes the contribution from the burner. Other peaks, if they occur, tend to appear much later. These early and late peaks represent differing stages in the involvement of the mock-up and will be discussed separately.

Heat Release During Burner Exposure. Figures 26a and 26b are bar graphs showing the peak heat release rate during the CB 133 gas burner exposure. The bars are clustered in accord with the fabric cover; within a cluster, the bars are labelled as to which barrier was used with the particular mock-up. The $80 \mathrm{~kW}$ limit set in the CB 133 protocol is indicated as a dashed line. Note that the data points shown in Table 4 as being approximate (due to equipment failures during a small number of tests) are not included in the Figure.

There are four replicate tests shown in Figures $26 \mathrm{a}$ and $26 \mathrm{~b}$. Two of these showed very good reproducibility (nylon and the heavy polypropylene with Barrier BB) and two showed a lesser reproducibility (light polypropylene and heavy polypropylene with Barrier $A$ ). The two test pairs showing the lesser reproducibility appear to do so for identifiable causes ${ }^{3}$ which were generally unique in their impact. Thus the relatively good level of reproducibility seen in the pairs of tests involving Sets 6 and 26 is believed to be representative of the test in general (for the first heat release peak) when all disruptive influences are minimized. These two sets have an average deviation of $\pm 3-4 \%$ about their mean results. Of course, one would have to have much more data on test reproducibility to make a definitive, statistically-based statement and it is likely that the

\footnotetext{
${ }^{3}$ In the case of one of the two tests with Set 16, the gas burner was inadvertently set $25 \mathrm{~mm}$ higher than the normal level; this slowed the involvement of the seat cushion and led to a substantially lower first heat release peak. In the case of one of the two tests with Set 25 , the gas burner flame was deflected partially to one side, possibly by the presence of an observer near that side of the mock-up. The ignition pattern was asymmetric, as was the subsequent burning, leading to a partial burnout on the seat back and a lower heat release peak. Thus in both of these cases, the more correct heat release peak is the larger of the two values measured. It is worth noting that these deviations suggest that the results of a CB 133 test are appreciably sensitive to factors affecting burner flame impingement on the surface of the test article.
} 
standard deviation obtained in this way would be larger than $\pm 3-4 \%$, but we have no further information.

Figure 26a suggests that for fabrics other than polypropylene, the fabric dominated the barrier in setting the level of the first heat release rate peak. The peak varied with fabric type by a factor of more than three in Figure 26a and the barrier impact was generally secondary. As in the Cone Calorimeter, it appeared that the principal material burning during the burner exposure was the fabric. However, in contrast to the Cone situation, with the thermoplastic fabrics, there were large areas (tenths of a square meter; e.g., sometimes nearly the entire seatback surface) on the inner surfaces of the mock-up which were bare, having had their fabric covering removed by melt/flow and/or curl-back as a response to flame impingement or approach. The bare areas were, of course, covered by the barrier, which in all cases remained intact during burner exposure. As noted in the discussion of the Cone data, the foam beneath this exposed barrier can be partially burned in response to the impingement of a heat flux, in this case from the igniter flames or from the spreading flames on the fabric. Figure 13 implies that this source of heat release was a fairly small complement to the fabric heat release rate. Furthermore, Figure 12 implies that it probably was rather short-lived, at least with Barriers $\mathrm{A}$ and $\mathrm{C}$. $^{4}$ As a result, the net effect of the tendency for fabrics to pull away from the flames and expose the barrier was probably one of lessening the heat release rate peak during the burner exposure. As in the Cone Calorimeter, the fabric which is "retreating" ends up as a thicker mass which burns more slowly but for a longer time. In the mock-ups, this longer burning sometimes resulted in further complications after the burner exposure, as discussed below. Needless to say, there is no assurance that the impact of these types of fabric splitting effects are quantitatively similar in the Cone and in the full-scale mock-ups.

In Figure 26b, where all of the fibers are polypropylene, one sees that the impact of the barrier under this material was not always secondary; it made a factor of two difference in two cases. Here the impact of the foam appears to have been secondary since the more highly retarded IFR foam has little consistent impact. The comments above regarding exposure of the barrier by "retreating" fabric apply here as well.

The results in Figures $26 \mathrm{a}$ and $\mathrm{b}$ are not very encouraging with regard to the issue of meeting the $80 \mathrm{~kW} \mathrm{CB} 133$ heat release rate limit; the majority of the material combinations tested exceed this limit. As noted earlier, this is in part a reflection of the choice of the four-cushion configuration, which tends to be a worst case due to radiative interaction among the four inner surfaces. A test with only two cushions, rather than four, shed some light on this; see below.

\footnotetext{
${ }^{4}$ All of the barriers showed an irregular tendency for an enhanced foam contribution on the vertical seatback surface. Wetted patches, generally small (tens of square centimeters), appeared on the seatback surface above the burner flame and became the attachment area for flames. They evidently were a result of random slumping of the foam against the inner surface of the seatback cushion. This type of enhancement of the heat release process is not captured in the Cone Calorimeter where the samples are horizontal, but it appears to be fairly minor in magnitude.
} 
Fire Growth Pattern During Burner Exposure. It is of interest to look more closely at the processes that occur during the burner exposure to gain further insight into the heat release process. Figure 27 is a typical result for what one sees in following the spread of flames on the inner surfaces of the mock-up during the burner exposure. The results are inferred from repeated viewings of the video tapes of the tests. The processes are similar on all mock-ups though the amount of surface area and the timing involved in a particular "step" varies.

Figure 27 shows that the gas burner leads first to convective ignition on the seat back and on the seat cushion. The ignited area on the seat back quickly enlarges as a result of upward spread of the flaming zone. The subsequent processes on the seatback get increasingly complex as angled upward spread gives way to splitting and curl back of the (thermoplastic) fabric, generally in an outward and upward direction. (The pure cotton fabric does not undergo any curl-back but its char does split open. The modacrylic-nylon fabric starts by splitting/curling but then chars and freezes in place.) Eventually the contracted segments of the thermoplastic fabrics melt and run downward while burning. If a segment is large enough, the melt will survive to reach the seat cushion where it continues to burn. Comparable processes are occurring on the inner surfaces of the arms but they start later because the heat flux to this region from the gas burner is less. The seat cushion surface undergoes lateral (opposed flow) type of flame spread processes, accompanied by splitting and curling back of thermoplastic fabrics; only the flow of melt is absent.

It is worth noting that the area in the corners where seat, seatback and arm surfaces come together ignites fairly abruptly (and rather late in the burner exposure) as a unit, apparently as a result of a predominantly radiative heat transfer process from the neighboring fire plumes. This is the area where one of the heat flux gages was located in most of the tests; see below.

Figure 28 shows the progression of the total area involved in the fire as a function of time for low, medium and high heat release rate material combinations. These data were again estimated from the video tapes of the tests. Two types of area are distinguished in this Figure. The area affected by the fire is the total area the fire has strongly affected (either by envelopment with attached flames or via fabric curl-back) at the given time; the area currently flaming is this large or less if some area elements are the result of curl-back (early in the burner exposure) or the result of burn-out (late in the exposure). With this in mind, one sees that the modacrylic-nylon combination has undergone extensive burn-out by 80 seconds as indicated by the large difference between the area affected by the fire and that burning at 80 seconds; cotton burn-out is much less and heavy polypropylene still less. Only this last has an appreciable contribution from fabric curl-back early in the burner exposure. Note that the peak flaming areas do differ significantly but the differences are not nearly enough to fully account for the differences in the peak rates of heat release shown in Figure 26. The total area of the inner surfaces of the mock-up is approximately $0.65 \mathrm{~m}^{2}$. The peak flaming area for the heavy polypropylene approached this. The modacrylic-nylon flaming area peaked at $0.44 \mathrm{~m}^{2}$. All of the other mock-ups gave intermediate values. Clearly then, at the peak the average rate of heat release per unit area for the polypropylene fabric mock-ups was substantially greater than that of, say, the modacrylicnylon mock-ups. 
Heat Fluxes During Burner Exposure. The heat flux data from the mock-up seat surface mimicked the rise and fall of the first heat release rate peak. Figure 29 shows a typical example. $^{5}$ Here both gages were in the rear corner region and were about $2 \mathrm{~cm}$ apart. The flux to the rearmost gage rose over the first minute of exposure to about $25 \mathrm{~kW} / \mathrm{m}^{2}$. In this time interval, the flames were growing on neighboring areas of the mock-up but were not impinging on this gage; thus the flux seen was primarily radiative. The flux then abruptly rose by more than a factor of two as the fabric around the gage ignited and direct convective heating supplemented the radiation from the near and more remote flames. It rose slightly more to its peak then fell abruptly as the burner was shut off and the radiating flame volume shrank.

One expects some correlation between the heat release peak and the spatially-averaged heat flux peak during the gas burner exposure. The heat flux feedback from the flame to the solid surfaces forces their gasification into fuel vapors which feed the heat release process. Figure 30 shows that there is indeed a rough correlation of the expected nature between the point measurements of heat flux and heat release rate but it is highly noisy. The correlation becomes even noisier if the ordinate is divided by the estimated area burning at the peak heat release time. Evidently the local flux does not accurately track the overall average feedback flux to the inner seat surfaces.

Table 5 shows all of the flux peak data. The rear gage is of primary interest, since it always indicates a peak flux level pertinent to the first heat flux peak; these are the data used in Figure 30. Note that the gage denoted as the front gage was placed in one of two locations depending on the test. In early tests it was very near the rear gage and both were in the area near the convergence of seat, seat back and arm cushion surfaces. In later tests the second gage was moved out to a location near the back edge of the gas burner where it saw generally lower fluxes and where the peak flux sometimes occurred after the burner was removed. Even when the two gages were very near each other (points in Table 5 with a single star), the peak flux readings were not always very close.

The front gage data points without a star in Table 5 are for the gage placed much further from the seatback, as noted above. These peak values were usually substantially lower than that seen by the rear gage indicating a decrease in flux away from the seatback. This explains why there was, in nearly all tests at the end of the gas burner exposure, unignited surface near the front of the chair, both on the seat and on the inner arm surfaces. Flame spread toward the front was slowed by the decreasing heat fluxes in this direction. This suggests that radiation from the

\footnotetext{
${ }^{5}$ Figure 29 is based on a data sampling rate of once every 10 seconds, as is the data in Table 5. In several of the tests the flux gage outputs were also recorded continuously on a chart recorder. In all cases the chart record showed a fluctuating signal with a period of seconds or less and an amplitude of roughly $\pm 10-15 \mathrm{~kW} / \mathrm{m}^{2}$ about the mean. The two recording modes gave substantially the same results for the peak flux except for Set \#13 where the chart record showed a brief spike to $93 \mathrm{~kW} / \mathrm{m}^{2}$.
} 
flames on the seatback and the hot seatback surface itself is a principal source of the increased fluxes at the back of the chair.

The peak fluxes in Table 5 are to be compared to those seen with the same material in the Cone Calorimeter (Figure 16). Recall that the fluxes in Figure 16 are the gage flux minus the external flux from the Cone calorimeter heater. The fluxes in Table 5 are the gage fluxes in the full-scale mock-ups. The difference between the results in Table 5 and in Fig. 16, for a given material combination, is an indication of the "effective external flux" in full-scale caused by radiation from the much larger flame volume and the hot cushion surfaces. Table 6 shows these estimates for three sets of materials. Two points should be noted. First, the effective external flux can be quite large, as least at the peak conditions shown here. Second, the effective external flux at the peak can vary with the material combination. Since this external flux is coming from the flame volume and surrounding hot surfaces and since these sources should vary with the peak heat release rate, flame temperature and soot volume fraction in the flame, it is to be expected that this effective external flux would vary, as well. Of course the effective external flux is strongly time-dependent here, as Figure 29 implies.

Correlation of First Peak With Cone Calorimeter. The rate of heat release at any time, $t$, from any spreading fire can be expressed as follows:

$$
Q(t)=\int_{0}^{t} R H R\left(t-t^{\prime}\right)\left(\frac{d A_{b}}{d t^{\prime}}\right) d t^{\prime} \ldots \ldots \ldots \ldots(I)
$$

Here $Q$ is in kilowatts, $\operatorname{RHR}\left(t-t^{\prime}\right)$ is the rate of heat release per unit area at time $t$ for an area element ignited at time $t^{\prime}$ and $\left(\mathrm{dA}_{\mathrm{b}} / \mathrm{dt}^{\prime}\right)$ is the time-varying rate of increase of burning area. This integral simply sums the heat release rate contributions at a given instant from all of the successively ignited portions of the burning object. It takes into account the fact that those elements which ignited first have been burning longer than later elements and thus are at a different point in their burning histories.

The role played by flame spread ( $\left(\mathrm{A}_{\mathrm{b}} / \mathrm{dt}^{\prime}\right.$ in Equation 1$)$ in setting the overall rate of heat release from a chair can be seen by considering the extremes of behavior. If the flame spread over the inner seat area instantaneously, this would be equivalent to igniting the entire area at once; the result would be a maximal heat release rate peak. If, on the other hand, the flame spread from the igniter area very slowly, the fabric would have time to burn out close behind the spreading flame front. A "ring" of flame would result, yielding a very low rate of heat release due to the minimal burning area. ${ }^{6}$ It is the rate of flame spread relative to the burning duration of the

\footnotetext{
${ }^{6}$ The behavior would differ in the absence of a barrier because the foam burning would persist much longer. Burnout is much less likely to play a role in furniture without barriers. Foam burning will play a correspondingly enhanced role.
} 
fabric which has a strong influence on the actual heat release peak. All of the real materials in the mock-ups used here are intermediate in this regard.

Evaluation of the integral in Equation (1) requires knowledge of the time-dependent terms within it and, in general, this knowledge is not available. However, if one assumes that the two functions can be replaced by their average values, evaluated between time zero and the time, $t_{p}$, of the heat release peak, Equation (1) becomes

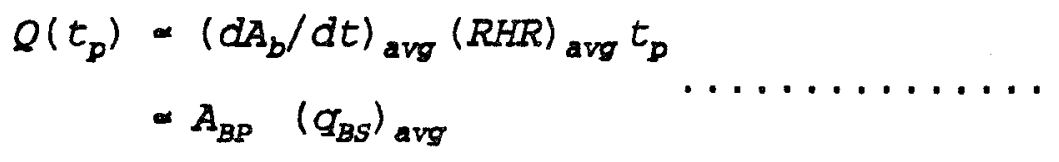

Note that the average rate of increase of burning area multiplied by the time of the peak reduces to the burning area at the peak, $\mathrm{A}_{\mathrm{BP}}$. The second approximate equality has replaced the average rate of heat release per unit area on the full-scale chair with the (hopefully) equivalent value measured in the Cone Calorimeter, $\left(\mathrm{q}_{\mathrm{BS}}\right)_{\mathrm{avg}}$. This last suggests a proportionality between Cone Calorimeter data and full-scale peak data. (The peak burning area is a variable as well but much less so than the rate of heat release behavior in the present study.) Such correlations with Cone data have been published previously [7].

It is of interest to see if the straightforward type of Cone to full-scale correlation used previously will work here. We thus seek a correlation between the highest heat release rate peak seen in the mock-up tests (over the entire test period) and the 180 second post-ignition average of the Cone Calorimeter heat release data. Figure 31a shows that this yields essentially no correlation here. This emphasizes the fact that the mechanism underlying the early and late heat release peaks in the mock-up tests is significantly different; it is best to focus on these peaks separately.

An attempt at a similar correlation between the Cone Calorimeter heat release data and the first heat release peak in the mock-up tests (during the burner exposure) is shown in Figure $31 \mathrm{~b}$. Here, since the gas burner exposure is only 80 seconds long and the earliest mock-up surface ignition requires roughly 20 seconds, a sixty second averaging time has been used for the Cone data (first $60 \mathrm{sec}$ after ignition). This is certainly more appropriate here than the 180 second averaging period used in Ref.10; here the 180 second averaged Cone data gives substantially more scatter than is seen in Figure 31b. The Cone data were all taken at the conventional external flux of $35 \mathrm{~kW} / \mathrm{m}^{2}$, as described above. ${ }^{7}$

\footnotetext{
${ }^{7}$ The correlation between the first full-scale heat release peak and the first Cone heat release peak was also examined; the results (not shown) are intermediate between those on Figures 31a and $31 b$.
} 
Clearly there is some correlation between the two types of data in Figure 31b. The correlation is quite noisy, however. The dashed lines suggest the outer limits of the data spread. Inspection of these implies an uncertainty of roughly $\pm 50 \%$ in full scale peak heat release rate using the indicated correlation line with a given average rate of heat release in the Cone Calorimeter. Figure 31b indicates that for the first heat release peak in a CB 133 test averaged Cone heat release data are only a coarse guide to expected full-scale performance. It is of interest to see if some better correlations can be established between small scale flammability parameters and full-scale fire performance of these mock-ups.

Dimensional Analysis of Fire Growth on a Chair. Dimensional analysis is a long-established, semi-empirical technique for obtaining useful expressions describing processes where the full governing equations are not available or are not tractable [11]. For example, it was the basis for nearly all heat and mass transfer correlations for many decades. Its use here is prompted by the encouraging efforts along these lines in the study of room-corner fire tests $[12,13]$, an analogous though somewhat simpler problem. Our goal in applying this technique will be to ascertain whether it can provide a simple but more complete guide than does average heat release rate in the Cone to the quantitative impact of varying the material combinations in a CB 133 test.

A key starting point for any dimensional analysis is knowledge of the component processes of the problem to be analyzed. Figure 27, derived from numerous video tapes of the growth of the fire during the CB 133 burner exposure, is the starting point here. Note that the analysis here is restricted to the fire during the burner exposure since other processes affect the later peak, as discussed below.

Figure 27 indicates that the key component processes involve convective and radiative ignition, upward and lateral flame spread and, finally, heat release rate subsequent to spread. All of these processes have been studied in other contexts and modelled to varying degrees $[9,14]$. However, it should be noted at the outset that the general fire growth problem on thermoplastic materials which undergo significant physical movements has not been studied. The probable consequences of this will emerge below.

Dimensional analysis requires only an ability to list the key parameters of the process to be analyzed. Recognizing the fundamental similarity between the present problem and the roomcorner problem of Refs. 12 and 13, we proceed analogously to those previous studies.

Both types of ignition processes noted above, as well as upward flame spread, respond principally to the thermal inertia of the fuel surface, denoted as (kpC), where $k$ is thermal conductivity, $\rho$ is density and $C$ is heat capacity of the fuel. In the case of layered materials like those here, these are effective values set by the depth of penetration of the thermal wave. A second parameter affecting these processes on a solid fuel is the ignition temperature, here denoted as $\Delta \mathrm{T}$, the difference between the actual ignition temperature and room temperature, where the latter is assumed constant. Finally, there is a characteristic heat flux from the flames to the solid, denoted as $\mathrm{q}_{\mathrm{ign}}$; this is taken to be a measure of that flux seen at all stages of the fire growth process, with no distinction between radiation and convection. Lateral flame spread introduces 
only one new parameter, a measure of the heat input at the attachment point of the spreading flame [14]. This is a distinctly different concept from $\mathrm{q}_{\mathrm{ign}}$; it is denoted as $\Phi$. The rate of heat release process of interest is that represented by the first peak in the Cone Calorimeter (principally due to the fabric). It will be characterized here by the height of the first Cone peak, $\mathrm{Q}_{\max }$, and the duration of the first peak, $\mathrm{t}_{B}$ (defined below). Finally, there is a characteristic length in this problem, the width of the gas burner, denoted as $l_{\mathrm{B}}$; all other lengths, e.g., seat, arm and seatback dimensions can be expressed as multiples of this length.

All of these parameters can be quantified in bench scale tests, in principle. The heat flux, $\mathrm{q}_{\mathrm{ign}}$ could be troublesome and ultimately, it will be replaced by another parameter below. Similarly, we will seek a substitute for the thermal inertia.

Once the key parameters are identified, dimensional analysis provides a formal procedure that allows one to re-cast the problem into a functional relationship between dimensionless groups of parameters. The procedure is straightforward, based on the necessity for dimensional consistency in any functional relationship [11]. The usual assumed form of a functional relationship is a power law. Using this procedure we proceed from

$$
Q(t)=f\left(t ; \Delta T, q_{1 g n},(k p C), \Phi, Q_{\max }, t_{B}, I_{B}\right) \ldots \ldots
$$

where $Q(t)$ is the total rate of heat release as a function of time. This becomes

$$
\frac{Q(t)}{Q_{\max }}=\left(\frac{(k p C)^{1 / 2} \Delta T}{\sqrt{t} Q_{\max }}\right)^{\alpha}\left(\frac{t_{B}}{t}\right)^{\phi}\left(\frac{Q_{1 g n}}{Q_{\max }}\right)^{\gamma}\left(\frac{\Phi}{Q_{\max }^{2} I_{B}}\right)^{\delta} \ldots \ldots
$$

This shows one of the principal advantages of dimensional analysis in multi-parameter problems: the number of independent parameters has been reduced, generally by the number of fundamental dimensions which the original parameters possess. It should be noted that a more general analysis of this specific problem would give rise to further dimensionless groups. These would involve test particulars such as the power output of the gas burner, exact placement of the burner, etc.; also there are further dimensionless groups involving the burner width to seat width, arm width, etc. Both types of groups are ignored here. The first because they are held constant by the test protocol; the second because they have not yet been varied experimentally.

The analysis does not produce values for the exponents in Equation (4); these must be determined by systematic experiments. It is worth noting that the exponents could be large or small, with the latter indicating a very weak dependence on the given dimensionless group. 
Equation 4, while certainly simpler than a full-blown model of the fire growth process, is still too complex for the practical goal of guiding users in judging the impact of materials substitutions. Furthermore, its applicability to real CB 133 data has not been demonstrated. As a practical matter, if all the dimensionless groups in this equation must be determined for each new material combination, it would probably be less expensive to simply run a CB 133 test. We are thus led to seek some further simplifications.

A surrogate for the numerator in the first dimensionless group of Equation 4 (thermal inertia times ignition temperature) is ignition delay time at a fixed flux. Cone data for this at $35 \mathrm{~kW} / \mathrm{m}^{2}$ is typically obtained during heat release testing. A surrogate for $\mathrm{q}_{\mathrm{ign}}$ in the third dimensionless group is the heat of combustion of the fabric, $Q_{\text {comb }}$; this is only a part of the source of heat flux variation with test materials, but it may suffice. The flame heat flux parameter, $\Phi$, has no ready surrogate.

With these ideas in mind, in combination with Equation 4, we arrive at the following proportionality:

$$
Q_{\text {peak }} \sim t_{1 g m}^{a} t_{B}^{b} Q_{\max }^{c} \Phi^{d} Q_{c o m b}^{0} \ldots \ldots \ldots(5)
$$

Once again, the exponents are to be determined by experiment. Note that we have moved up from four parameter groups to five independent parameters. This expression can be turned into an empirical equation if an experimentally-determined constant is placed in front of the right hand side. We note that four of the five materials parameters are available from Cone tests. Note also that Equation 5 is no longer in dimensionless form.

It was noted previously that not all of the materials parameters introduced into this analysis are necessarily of significant importance or sufficiently variable with the materials tested to play a role here. Since we do not have a measure of the lateral spread parameter, $\Phi$, for the materials in this study, we will proceed as if it was sufficiently invariant to be lumped into the constant of proportionality in Equation 5. (The value of $\Phi$ is obtainable from LIFT, i.e., ASTM E-1321, tests but these results are not available for the materials in this study.)

The next step in testing the validity of the above approach is to attempt to fit Equation 5 (with a proportionality constant) to the available Cone and full-scale data. This was done here using a non-linear, least-squares fitting routine available within the Sigmaplot software package. The subset of data for charring fabrics (cotton, modacrylic-nylon, polyester-cotton) comprises the group that most closely follows the assumptions here as to controlling mechanisms in the fire growth. These fabrics largely stay in place during the flame spread processes. This yields the closest correspondence between their behavior in the Cone and that in CB 133 during the burner exposure. 
Figure 32a shows the correlation between Equation 5 and full-scale data for the charring fabrics. Perfect agreement would mean that all of the experimental points lay on the dashed line. Agreement for these material sets is fairly good, in spite of the omission of the factors $\Phi$ and Q comb'

Figure $32 \mathrm{~b}$ shows the correlation for the full data set based on the same three materials parameters as for the charring fabrics; it clearly is much less convincing, though slightly better than Figure 31b, based on the averaged Cone data for heat release rate alone. Note that these two correlations use three of the parameters available from the Cone Calorimeter. Figure 32c shows the correlation for full data set when the heat of combustion of the material combination is also factored into the statistical determination of model parameters. This tightens the correlation for most of the data points but it leaves two points well out of line. Unfortunately, the out-of-line points fall to the high side of the correlation line meaning that the correlation prediction would underestimate the hazard of the specific material combinations these point represent. Both of those points, one for the Light PP/ Barrier A/ IFR Foam combination and one for PE/ Barrier B/ Cal 117 Foam, seem anomalously high with regard to full-scale heat release rate when compared to the same fabric and foam with other barriers; however, there is no known anomaly in the tests which would allow one to discard the results.

Note that the statistical model parameters are substantially different in Figures $32 \mathrm{a}, \mathrm{b}$ and $\mathrm{c}^{8}$ The parameters must be viewed as strictly descriptive of only the limited and rather noisy data currently available; they cannot be assigned any physical significance at present. There is a distinct need to apply this approach first to a larger data set of charring fabrics in order to obtain a more definitive assessment of its general applicability.

For thermoplastic fabrics, Equation 5 is less successful for two possible reasons. The role of the parameter $\Phi$ may be more significant, in that it may vary more among these fabrics. There are no data with which to judge this issue at present. A possibly more significant reason is that fire growth on thermoplastic-covered furniture is inherently more complex. As was noted previously, these fabrics tended to pull strongly away from the burner flame, leaving substantial fractions of the cushion surfaces devoid of fabric on a scale that is not modelled by Cone behavior; such pullback continues as the fire grows further. The burn duration then varied from very short where only barrier and foam were exposed to extra long where fabric melt accumulations occurred. It is doubtful that the full complexity of this behavior can be accurately modeled from first principles. On the other hand, the type of semi-empirical model which Equation 5 represents

\footnotetext{
${ }^{8}$ One contributor to the differences is that two different measures of the duration of the first heat release peak in the Cone have been used. "Peak Time" is the duration from ignition to the bottom of the valley between peaks. When there was no second peak, the endpoint was taken as the time when the RHR had fallen to $10 \%$ of the peak value. "Half Peak Time" is the time duration of the first peak at half of its peak height. Where a given measure is shown in the labelling of the abscissa, it was chosen because it appeared to give a better fit than the alternative definition.
} 
may capture it sufficiently if a proper measure of the fabric movement tendency can be included as a variable. Such a measure might be obtained in LIFT-like (i.e., ASTM 1321, lateral flame spread test), heat flux gradient exposures of the fabric/barrier combination. An average value of the exposed barrier area for horizontal and vertical sample orientations could perhaps become the needed additional variable. This requires experimental exploration.

Effect of Mock-Up Geometry. Note in Figure 17, for PE/ Barrier A/Cal 117, there was a second test run that included only two cushions rather than the complete complement of four cushions. The arm cushions were omitted here to provide a small amount of exploration of mock-up geometry effects. The effect here is dramatic. The burn area is reduced to little more than that below the burner and an area slightly wider than the burner on the full height of the seatback. This is about a factor of two less than the peak burning area with four cushions. The lack of ignition on the inner arm surfaces seems to have a very substantial impact on the spread of flame across the seat area and on the outer portions of the seatback. This suggests that there could be a fairly abrupt decrease in peak rate of heat release for chairs with arms if the seat width is increased by only several centimeters. The heat flux from the side flames on the gas burner has not been assessed directly but it probably falls off rapidly just beyond the flame tips. For the seat cushion width used here, the side flame tips just barely reach the side arms during the burner exposure. Of course, the hazard that a chair represents in the real world would not be decreased by a slight increase in seat width. Issues such as this will be explored further in the planned follow-on study of chair geometry effects.

Heat Release After Burner Exposure. Rarely did the fire extinguish in close proximity to the time of termination of the burner exposure. Inspection of Figures 17 to 25 shows that typically some heat release continued for an extended period. Typically this was prompted by continued flame spread on the fabric in regions which were favorable to this process, e.g., along crevices formed by the juncture of two or more cushions.

A particularly favorable pathway for continued flame spread was into the "tunnels" formed by the juncture of the back, seat and arm cushions. (In the lefthand side of Figure 1, these tunnels lie at the lower, outer corners of the seatback as it sits on the seat cushion.)This space was roughly triangular in cross-section with a characteristic dimension of the order of 1 to $2 \mathrm{~cm}$. It constituted a pathway from the inner seat area of the mock-up to the rear of the mock-up, at the base of the seat back. It is worth noting that this path led in this direction because the seatback cushion rested on top of the seat cushion. If the back cushion instead rested behind the seat cushion, a tunnel would still exist on each side but it would lead to the bottom, not the back, of the mock-up. It is not known to what extent this type of detail influenced the further development of the fire; it is probable that much of what is discussed below would have still occurred if the cushion placement was re-arranged in this manner.

Such "tunnels" will exist in any chair that has discrete seat and back cushions as well as arms. However, it is not clear that the consequences of flame propagation through the tunnels of actual chairs are the same as they are in this mock-up. Here the space the tunnels lead to is open so that the emerging flames can grow without any limitations as to available oxygen. Some realistic 
chair designs would probably inhibit growth of the emerging flames because they would encounter structural elements of the chair.

An attempt was made to see if propagation through these tunnels could be stopped. This was done with the duplicate cushions of Sets 2,6 and 26. The tunnels were plugged with masses of ceramic wool prior to burner exposure. In all three cases, the only effect was to slow the spread of the flames through these pathways. Though the tunnels were always fully blocked initially, they were re-opened by shrinkage of the seatback cushion, subsequent to the burner exposure, as a result of continued burning in the crevice regions on the front of this cushion. The net result of the test was never altered by plugging the tunnels.

Figures 17 to 25 indicate that there often was a second heat release peak subsequent to the termination of the gas burner; usually, if it occurred, the second peak was higher than that during the burner exposure. At least one significant second peak was seen with every fabric except the modacrylic-nylon blend.

Figures $33 \mathrm{a}$ and $\mathrm{b}$ summarize the post-burner peak values for the various material combinations. In Figure 33a the variation of largest peak height with fabric is similar to that in Figure 26a, which shows the behavior during the gas burner exposure. Here, however, there is a much greater dependence on the type of barrier. Note particularly that for fabrics other than polypropylene, Barrier $C$ suppressed any significant post burner heat release peaks. Barrier B was markedly less successful. This is the barrier which allowed flame penetration in some tests. On the other hand, its flame-retarded counterpart, Barrier BB, was able to suppress significant post-burner peaks for polypropylene (with FR backcoating) over both Cal 117 and IFR foams (Fig. 33b).

Figure 33b shows a case of very poor reproducibility with Barrier A over IFR foam (Set 25). The reason has to do with the tendency for arm cushions to shrink and fall out of the mock-up frame onto the floor. This is what happened in an early test for the case showing a very high heat release peak $(419 \mathrm{~kW})$. After the short wires were added under the ends of the arm cushions to prevent them from falling, the second result was obtained (108 kW). The cushion which fell in the first case landed under the seat and acted like a severe floor fire interacting with the remaining cushions. The highest peak in Fig. $33 \mathrm{~b}(744 \mathrm{~kW})$ occurred for similar reasons, early in the test program. Floor fires caused by accumulated polyurethane foam melt contributed to the severity of other large peaks, even though the test cushions stayed in place.

Barrier Failure Mechanism. In every case where the second peak was significant (greater than, say, $50 \mathrm{~kW}$ ), it appeared to be a result of the same mechanism. That mechanism, which we call a "basal melt fire" is illustrated by the sketch in Figure 34. In the Figure flames have become established on the bottom of a vertically-oriented cushion whose barrier is completely intact; this might be the seatback or an arm cushion. The fuel for the flames was initially the fabric but it has increasingly become the "melt" from the polyurethane foam; this melt is flowing down from the lateral surfaces of the cushion and out through the pores in the barrier at the base of the cushion. A continuing supply of melt is assured by the transfer of heat from the flames lapping 
on the sides of the cushion; the heat is transferred by convection and radiation to the degrading surface of the foam within the barrier. With Barrier B this mechanism was sometimes abetted by the appearance of flames within the confines of the barrier of at least one cushion.

The sketch in Figure 34 implies a steady-state fire. Actually this sort of situation probably existed in every test to some degree at some point in time. With some material combinations, this type of fire died out because the heat transfer to the foam became increasingly inadequate as the foam surface retreated inward away from the barrier surface. With other cases the fire tended to grow unsteadily until a substantial amount of the foam melt dripped though the basal flames on the cushion, starting a fire on the floor. The highest heat release rate peaks occurred when the floor fire became large enough to interact strongly with the mock-up fire above it. Even in the absence of an interacting floor fire, this general mechanism could strip all of the urethane foam out of the cushions, burning most of it, without having to break the barrier.

Inspection of Figures 17 to 25 indicates that the polypropylene fabrics were most prone to this fire mechanism. They also tended to succumb to this type of failure sooner than the other fabrics. In light of the suggested mechanism above, it is probable that this is in large measure a result of their higher heat of combustion (bomb calorimeter value of $11,080 \mathrm{cal} / \mathrm{g}$; Ref. 15). This was supplemented by a tendency for the fabric on the seatback to melt and flow down to the seat during the burner exposure, forming a longer-lasting pool fire there which could initiate and help sustain the above mechanism.

For the other fabrics, the type of barrier tended to be more important, suggesting that the heat transfer through the barrier was more marginal because of their lower heats of combustion. Nylon, which has a fairly high heat of combustion (bomb calorimeter value for nylon 66,7580 $\mathrm{cal} / \mathrm{g}$; Ref. 15) and a tendency similar to polypropylene to melt and run down from the seatback, yielded large fires with both Barriers A and B.

Barrier A led to three large basal melt fires whereas Barrier B led to two. There is very likely some chance element to this type of fire mechanism so that this difference may not be significant. Barrier $\mathrm{C}$ led to such a fire only in combination with a polypropylene fabric.

It is unclear whether the CB 133 mock-up is more vulnerable to this fire mechanism than are real chairs. For polypropylene fabrics, it appeared that the basal melt fire could originate from the juncture of the top of the seat and the front of the seatback. More commonly with this and other fabrics it appeared to originate from the area on the back of the mock-up where the these same two cushions met or from the area below the rear of the "tunnels" described above. Thus either the seatback cushion or an arm cushion could be the bearer of a growing basal melt fire.

The suggested mechanism of this type of fire also implies some means to avoid it. First of all, chairs which have continuous padding from arms to back and lack a discrete seat cushion should be invulnerable because there is no ready way for the flames to get established at the base of an encased block of foam. An earlier study in this laboratory using generally similar materials but in the form of chairs with "tight" seat, back and arm cushions (i.e., the cushioning was integral 
with the chair support structure) gave no second heat release peak at all. Chairs which are close analogs of the discrete cushion design tested here could be made more resistant by use of a fireresistant layer over the structure which directly underlies the seat and back cushions. This would severely restrict the air supply to "tunnel" areas where basal melt fires are most likely to originate. Chairs of this design may still be vulnerable if covered in a heavy polypropylene fabric, since this combination appeared to allow this fire mechanism to originate at the front bottom edge of the seat back cushion. More effective thermal insulation (e.g., a doubled barrier layer) in this area of such chairs would probably be necessary.

Correlation With Cone Calorimeter. It is of interest to know whether the results of Cone Calorimeter testing are predictive of basal melt fires. Given the suggested mechanism and the apparent role of chair configuration, it would be rather surprising if they were. Figure 35 shows an attempt at a simple correlation between the second heat release rate peak in the mock-up tests and the 60 second average result from the Cone Calorimeter at $35 \mathrm{~kW} / \mathrm{m}^{2}$. The 60 second average is used here because it looks somewhat more convincing than an attempted correlation with Cone data averaged over 180 seconds. The former emphasizes the fabric behavior, as noted above. Inspection of Figure 35 shows that there clearly is no simple proportionality between the data on the two scales. At best, the results suggest a threshold for the Cone data ( $\mathrm{ca} .125$ $\mathrm{kW} / \mathrm{m}^{2}$ ) above which one might get a second large heat release peak in the mock-up test. (Recall that the type of barrier counts with non-polypropylene fabrics.) This uncertainty in the likely outcome makes this observation of limited practical use, especially since only the modacrylicnylon fabric fell consistently below this threshold. It appears that one must pay close attention to the design features that can facilitate basal melt fires in order to be assured of avoiding them.

The same general approach used above on the first heat release peak can presumably provide a possible basis for correlating the severity of the second heat release peak. From the above discussion, two relevant parameters in a dimensional analysis would be the heat of combustion of the fabric and the thermal resistance of the barrier. A quantitative measure of this latter quantity is not currently available; it is needed for the mixed convective/radiative conditions of the fire tests. It is likely that further analysis will reveal other relevant parameters in need of quantification. The need for further work in this area is evident.

\section{4) Summary and Conclusions}

The results of this study are mixed. Many of the details of furniture mock-up performance in the CB 133 protocol are clearer but we have yet to provide a definitive bench-scale basis for predicting this performance.

A set of full-scale data has been developed for a fixed but rather severe chair geometry. This data set is of some direct use as a basis for judging which material substitutions will move a failing chair design toward a passing chair design in the CB 133 test. Of course, the number of fabrics examined is minuscule compared to the possible choices in the real world. 
Most fabrics are blends of two or more of the types of fibers used here but there are no rules available to predict how fiber blends will perform, given the performance in the pure fiber cases. Indeed, since the performance of a blend will depend on fabric structure as well as fiber content, and the number of possible variants here is enormous, it is doubtful that there will ever be a useful set of rules for "blended" fire performance. This is one of the principal reasons for placing so much hope in the Cone Calorimeter. This device can be used to get measures of bench-scale fire performance for any fabric; the process is indifferent to material composition.

The current work has sought to extend previous indications of the usefulness of Cone data to the new conditions of ignition exposure and material combinations imposed by CB 133. The results are still somewhat unclear, due largely to the limited data set. It is clear that a simple time average of the rate of heat release seen in the Cone is not an adequate predictor of CB 133 performance. The Cone provides several other pieces of information which appear to be relevant. These seem to improve the bench-scale to full-scale correlation but it is possible that, for thermoplastic fabrics, more information is needed to fully capture the pertinent behavioral parameters of the full-scale test. Application of the data analysis procedures described here to larger sets of Cone and full-scale data should provide a definitive answer to the value of this approach.

The whole domain of geometric variables in furniture design remains to be examined. These are known to be of equal importance to material choices. A systematic variation of cushion dimensions in the CB 133 mock-up is the next logical step in this study and will be pursued in the near future. The study will yield a data set sufficiently broad to be useful in itself as guidelines to manufacturers. We will also seek to extend the dimensional analysis approach along these lines. 


\section{$\underline{\text { References }}$}

1) California Bureau of Home Furnishings and Thermal Insulation, Technical Bulletin 133 Information Package, January, 1992

2) Quintiere, J., "Furniture Flammability: An Investigation of the California Technical Bulletin 133 Test. Part 1: Measuring the Hazards of Furniture Fires", National Institute of Standards and Technology NISTIR 4360, July, 1990

3) Ohlemiller, T. and Villa, K., "Furniture Flammability: An Investigation of the California Technical Bulletin 133 Test. Part 2. Characterization of the Ignition Source and a Comparable Gas Burner", National Institute of Standards and Technology NISTIR 4348, June, 1990

4) Parker, W. And Tu, K.-M., "Furniture Flammability: An Investigation of the California Technical Bulletin 133 Test. Part 3. Full Scale Chair Burns", National Institute of Standards and Technology NISTIR 4375, July, 1990

5) Barile, P., "A Systematic Approach for Predicting Compliance with Technical Bulletin 133 for Untested Combinations of Chair Styles and Fabrics", Paper presented at the Eighteenth International Conference on Fire Safety, San Francisco, CA, January, 1993

6) Nemschoff, M., "California Technical Bulletin 133 - A Furniture Manufacturer's Perspective", Paper presented at the Nineteenth International Conference on Fire Safety, San Francisco, CA, January, 1994

7) Ames, S. Babrauskas, V. and Parker, W., "Upholstered Furniture: Prediction by Correlations", in Heat Release in Fires, Babrauskas, V. and Grayson S. (eds.), Elsevier Applied Science, New York (1993), p. 520

8) Babrauskas, V. and Wetterlund, I., "Fire Testing of Furniture in the Cone Calorimeter - The CBUF Test Protocol", Swedish National Testing and Research Institute, Boras, Sweden, SP Report 1994:32

9) Quintiere, J. and Rhodes, B., "Fire Growth Models for Materials", University of Maryland, Fire Protection Engineering Department, Final report to NIST on Grant 60NANB2D1266, January, 1994

10) Babrauskas, V., "Bench-Scale Methods for Prediction of Full-Scale Fire Behavior of Furnishings and Wall Linings", Society of Fire Protection Engineers Technology Report 8410, Society of Fire Protection Engineers, Boston MA, 1984 
11) Zlokarnik, M., Dimensional Analysis and Scale-Up in Chemical Engineering, SpringerVerlag, Berlin, 1991

12) Thomas, P. and Karlsson, B., "Dimensionless Quantities in Fire Growth: The Weighting of Heat Release Rate", Department of Fire Safety Engineering, Lund University, Lund, Sweden, December, 1991

13) Kokkala, M., Thomas, P. and Karlsson, B., "Rate of Heat Release and Ignitability Indices for Surface Linings", Fire \& Materials, 17, No. 5 (1995) p. 209

14) Society of Fire Protection Engineers, SFPE Handbook of Fire Protection Engineering, National Fire Protection Association, Quincy, MA (1988), Chapters 1-21 and 1-24

15) Domalski, E., Evans, W. and Jobe, T., "Thermodynamic Data for Waste Incineration", National Bureau of Standards NBSIR 78-1479, August, 1978 
Table 1

Description of Materials

\begin{tabular}{|c|c|c|}
\hline $\begin{array}{c}\text { Fabric } \\
\text { Designation }\end{array}$ & Fiber Composition & $\begin{array}{l}\text { Areal Density } \\
(\mathrm{g} / \mathrm{m} 2-\text { oz/yd2) }\end{array}$ \\
\hline PE & $100 \%$ polyester & $300-9$ \\
\hline Nylon & $100 \%$ nylon & $390-11.5$ \\
\hline MA-N & $75 \%$ modacrylic/25\% nylon & $370-11$ \\
\hline PE-Cot & $38 \%$ polyester $/ 62 \%$ cotton & $300-9$ \\
\hline Cot & $100 \%$ cotton & $300-9$ \\
\hline Lt PP & $100 \%$ polypropylene & $270-8$ \\
\hline Hvy PP & $\begin{array}{c}100 \% \text { polypropylene } \\
\text { w. FR backcoat }\end{array}$ & $410-12$ \\
\hline $\begin{array}{c}\text { Foam } \\
\text { Designation }\end{array}$ & Foam Composition & $\begin{array}{c}\text { Foam Density } \\
(\mathrm{kg} / \mathrm{m} 3-\mathrm{lb} / \mathrm{ft} 3)\end{array}$ \\
\hline Cal 117 & $\begin{array}{c}\text { polyurethane } \\
\text { w. low level halogen FR }\end{array}$ & $31-1.9$ \\
\hline IFR & $\begin{array}{c}\text { polyurethane } \\
\text { w. med. level melamine }\end{array}$ & $33-2.0$ \\
\hline $\begin{array}{c}\text { Barrier } \\
\text { Designation }\end{array}$ & $\begin{array}{c}\text { Barrier } \\
\text { Composition }\end{array}$ & $\begin{array}{l}\text { Areal Density } \\
(\mathrm{g} / \mathrm{m} 2-0 \mathrm{z} / \mathrm{yd} 2)\end{array}$ \\
\hline A & non-woven aramid fiber & $68-2.0$ \\
\hline B & $\begin{array}{l}\text { knitted glass/ } \\
\text { charring fiber }\end{array}$ & $250-7.3$ \\
\hline BB & $\begin{array}{l}\text { B embedded w. } \\
\text { halogen FR resin }\end{array}$ & $470-13.8$ \\
\hline $\mathrm{C}$ & woven glass fiber & $120-3.5$ \\
\hline
\end{tabular}


Table 2

Material Combinations Used in Testing

\begin{tabular}{|c|c|}
\hline Set Number & Fabric/Barrier/Foam \\
\hline 1 & Polyester/Barrier A/Cal 117 \\
\hline 2 & $"$ /Barrier B/ " \\
\hline 3 & " /Barrier C/ " \\
\hline 4 & Nylon/Barrier A/Cal 117 \\
\hline 5 & " /Barrier B/ n \\
\hline 6 & " /Barrier C/ " \\
\hline 7 & Modacrylic-Nylon/Barrier A/Cal 117 \\
\hline 8 & $n \quad$ Barrier B/ $n$ \\
\hline 9 & $" \quad /$ Barrier $\mathrm{C} / n$ \\
\hline 10 & Polyester-Cotton/Barrier A/ Cal 117 \\
\hline 11 & " /Barrier B/ " \\
\hline 12 & $" \quad /$ Barrier $\mathrm{C} / \quad "$ \\
\hline 13 & Cotton/Barrier A/Cal 117 \\
\hline 14 & $"$ /Barrier B/ " \\
\hline 15 & $"$ Barrier Cl " \\
\hline 16 & Light Polypropylene/Barrier A/Cal 117 \\
\hline 17 & $" \quad$ Barrier B/ $"$ \\
\hline 18 & $" \quad$ Barrier $\mathrm{C} / n$ \\
\hline 19 & Light Polypropylene/Barrier A/FR Foam \\
\hline 20 & " /Barrier B/ $"$ \\
\hline 21 & $" \quad$ Barrier $\mathrm{C} / "$ \\
\hline 22 & Heavy Polypropylene/Barrier A/Cal 117 \\
\hline 23 & " $\quad$ Barrier BB/ " \\
\hline 24 & $" \quad$ Barrier C/ $"$ \\
\hline 25 & Heavy Polypropylene/Barrier A/IFR Foam \\
\hline 26 & " /Barrier BB/ " \\
\hline 27 & $" \quad$ Barrier Cl " \\
\hline
\end{tabular}


Table 3

Time-Averaged Cone Calorimeter Results (Avg. \pm Avg. Dev.)

\begin{tabular}{|c|c|c|c|}
\hline $\begin{array}{l}\text { Set Num- } \\
\text { ber }\end{array}$ & Materials & $\begin{array}{c}60 \text { Second } \\
\text { Avg. } \text { RHR }(\mathrm{kW} / \mathrm{m} 2)\end{array}$ & $\begin{array}{c}180 \text { Second } \\
\text { Avg. } \operatorname{RHR}(\mathrm{kW} / \mathrm{m} 2)\end{array}$ \\
\hline 1 & $\mathrm{PE} / \mathrm{A} / \mathrm{Cal} 117$ & $128 \pm 0.9$ & $82 \pm 16.1$ \\
\hline 2 & $" / \mathrm{B} / "$ & $139 \pm 5.3$ & $154 \pm 5.0$ \\
\hline 3 & $" / \mathrm{C} / "$ & $96 \pm 6.7$ & $32 \pm 2.0$ \\
\hline 4 & Nylon/ A /Cal 117 & $211 \pm 6.2$ & $132 \pm 8.0$ \\
\hline 5 & $" / \mathrm{B} / "$ & $263 \pm 4.4$ & $203 \pm 9.7$ \\
\hline 6 & $" / \mathrm{C} / "$ & $204 \pm 1.8$ & $71 \pm 0.4$ \\
\hline 7 & MA-N/A /Cal 117 & $86 \pm 15.5$ & $39.5 \pm 10.5$ \\
\hline 8 & $" / \mathrm{B} / "$ & $82 \pm 9.4$ & $44 \pm 7.5$ \\
\hline 9 & $" / \mathrm{C} / "$ & $53 \pm 6.3$ & $18 \pm 2.7$ \\
\hline 10 & PE-Cot/A /Cal 117 & $201 \pm 4.2$ & $114 \pm 1.1$ \\
\hline 11 & $" / \mathrm{B} / "$ & $173 \pm 4.4$ & $143 \pm 3.3$ \\
\hline 12 & $n / \mathrm{C} / n$ & $113 \pm 2.7$ & $38 \pm 1.1$ \\
\hline 13 & Cot/ A / Cal 117 & $175 \pm 7.0$ & $100 \pm 2.0$ \\
\hline 14 & $" / \mathrm{B} / "$ & $178 \pm 8.7$ & $143 \pm 3.0$ \\
\hline 15 & $" / \mathrm{C} / n$ & $113 \pm 9.1$ & $51 \pm 5.7$ \\
\hline 16 & Lt PP/ A /Cal 117 & $234 \pm 2.2$ & $162 \pm 6.3$ \\
\hline 17 & $" / \mathrm{B} / n$ & $137 \pm 5.1$ & $147 \pm 9.0$ \\
\hline 18 & $" / \mathrm{C} / "$ & $133 \pm 0.9$ & $45 \pm 0.7$ \\
\hline 19 & Lt PP/ A / IFR Foam & $225 \pm 7.3$ & $139 \pm 5.3$ \\
\hline 20 & $" / \mathrm{B} / "$ & $130 \pm 5.1$ & $129 \pm 6.3$ \\
\hline 21 & $" / \mathrm{C} / "$ & $127 \pm 9.8$ & $49 \pm 8.3$ \\
\hline 22 & Hvy PP/ A /Cal 117 & $256 \pm 4.7$ & $189 \pm 9.2$ \\
\hline 23 & $" / \mathrm{BB} / "$ & $169 \pm 4.7$ & $89 \pm 15$ \\
\hline 24 & $n|\mathrm{C}| "$ & $133 \pm 16.2$ & $48 \pm 5.0$ \\
\hline 25 & Hvy PP/ A / IFR Foam & $226 \pm 9.5$ & $144 \pm 3.0$ \\
\hline 26 & $" / \mathrm{BB} / "$ & $145 \pm 4.9$ & $94 \pm 9.2$ \\
\hline 27 & $" / \mathrm{C} / "$ & $131 \pm 8.2$ & $51 \pm 4.3$ \\
\hline
\end{tabular}


Table 4

Heat Release Peaks From Full-Scale Mock-Up Burns

\begin{tabular}{|c|c|c|c|}
\hline $\begin{array}{c}\text { Set } \\
\text { Number }\end{array}$ & Materials & $\begin{array}{c}\text { Peak RHR During } \\
\text { Burner Exposure } \\
\text { (kW) } \\
\text { (At Time, s) }\end{array}$ & $\begin{array}{c}\text { Peak RHR After } \\
\text { Burner Exposure } \\
\text { (kW) } \\
\text { (At Time, s) }\end{array}$ \\
\hline 1 & $\mathrm{PE} / \mathrm{A} / \mathrm{Cal} 117$ & $60(128 s)$ & $83(938 \mathrm{~s})$ \\
\hline 2 & $" / \mathbf{B} / \quad "$ & $84(138 s)$ & $20(640 \mathrm{~s})$ \\
\hline 3 & $" / \mathrm{C} / "$ & $100(128 \mathrm{~s})$ & $12(750 \mathrm{~s})$ \\
\hline 4 & Nylon/ A /Cal 117 & ca. $100^{9}$ & ca. $300^{2}$ \\
\hline 5 & $" / \mathrm{B} / "$ & $141(138 s)$ & $476(1098 s)$ \\
\hline 6 & $" / \mathrm{Cl} "$ & $\begin{array}{l}135(139 \mathrm{~s}) \\
147(138 \mathrm{~s})\end{array}$ & $\begin{array}{c}32(900 \mathrm{~s}) \\
<20(860 \mathrm{~s})\end{array}$ \\
\hline 7 & MA-N/A /Cal 117 & ca. $35^{2}$ & None \\
\hline 8 & $" / \mathrm{B} / n$ & $35(128 \mathrm{~s})$ & None \\
\hline 9 & $n / \mathrm{C} / n$ & $35(138 \mathrm{~s})$ & None \\
\hline 10 & PE-Cot/ A /Cal 117 & ca. $60^{2}$ & ca. $40^{2}$ \\
\hline 11 & $" / \mathrm{B} / "$ & $177(108 \mathrm{~s})$ & $326(1808 \mathrm{~s})$ \\
\hline 12 & $" / \mathrm{C} / n$ & 117 (118 s) & $22(370 \mathrm{~s})$ \\
\hline 13 & Cot/ A /Cal 117 & $66(138 s)$ & $106(958 \mathrm{~s})^{10}$ \\
\hline 14 & $" / \mathbf{B} / "$ & $90(128 \mathrm{~s})$ & $32(1138 s)$ \\
\hline 15 & $" / \mathrm{C} / "$ & $70(125 \mathrm{~s})$ & $<10(640 \mathrm{~s})$ \\
\hline 16 & Lt PP/ A /Cal 117 & $\begin{array}{c}109(199 \mathrm{~s})^{3} \\
151(148 \mathrm{~s})\end{array}$ & $\begin{array}{l}378(670 \mathrm{~s})^{3} \\
242(478 \mathrm{~s})\end{array}$ \\
\hline 17 & $" / \mathrm{B} / "$ & $130(138 \mathrm{~s})$ & $188(668 \mathrm{~s})$ \\
\hline 18 & $" / \mathrm{C} / "$ & $150(128 \mathrm{~s})$ & $744(648 s)$ \\
\hline 19 & Lt PP/ A / /FR & $235(118 \mathrm{~s})$ & $272(538 \mathrm{~s})$ \\
\hline 20 & $n / \mathrm{B} / n$ & $96(138 \mathrm{~s})$ & $229(598 s)$ \\
\hline 21 & $n / \mathrm{C} / n$ & $136(128 \mathrm{~s})$ & $326(1048 \mathrm{~s})$ \\
\hline
\end{tabular}

${ }^{9}$ Visual estimate only; RHR system failure.

${ }^{10}$ Peak possibly enhanced by failure of the seat cushion zipper. 
Table 4, Cont'd

Heat Release Peaks From Full-Scale Mock-Up Burns

\begin{tabular}{|c|c|c|c|}
\hline $\begin{array}{c}\text { Set } \\
\text { Number }\end{array}$ & Materials & $\begin{array}{c}\text { Peak RHR During } \\
\text { Burner Exposure } \\
\text { (kW) } \\
\text { (At Time, s) }\end{array}$ & $\begin{array}{c}\text { Peak RHR After } \\
\text { Burner Exposure } \\
\text { (kW) } \\
\text { (At Time, s) }\end{array}$ \\
\hline 22 & Hvy PP/ A /Cal 117 & $175(128 \mathrm{~s})$ & $290(598 \mathrm{~s})$ \\
\hline 23 & $" / \mathrm{BB} / "$ & $82(138 \mathrm{~s})$ & $57(1438 \mathrm{~s})$ \\
\hline 24 & $" / \mathrm{C} / "$ & $128(138 \mathrm{~s})$ & $473(1038 \mathrm{~s})$ \\
\hline 25 & Hvy PP/ A /FR & $\begin{array}{l}112(108 \mathrm{~s}) \\
165(138 \mathrm{~s})\end{array}$ & $\begin{array}{c}419(828 \mathrm{~s}) \\
108(1169 \mathrm{~s})\end{array}$ \\
\hline 26 & $" / \mathrm{BB} / n$ & $\begin{array}{l}98(138 \mathrm{~s}) \\
92(120 \mathrm{~s})\end{array}$ & $\begin{array}{l}35(1088 \mathrm{~s}) \\
23(4900 \mathrm{~s})\end{array}$ \\
\hline 27 & $" / \mathrm{C} / "$ & $144(138 \mathrm{~s})$ & $72(1318 \mathrm{~s})$ \\
\hline
\end{tabular}

*Time in all of the tests began 60 seconds before the gas burner was ignited so that background data could be collected. Thus the burner exposure ended at 140 seconds in the time frame used here. 
Table 5. Peak Heat Flux During Bumer Exposure

\begin{tabular}{|c|c|c|c|}
\hline $\begin{array}{c}\text { Set } \\
\text { Number }\end{array}$ & Materials & $\begin{array}{c}\text { Rear Gage } \\
\text { Peak Flux } \\
\left(\mathbf{k W} / \mathbf{m}^{\mathbf{2}}\right)\end{array}$ & $\begin{array}{c}\text { Front Gage } \\
\text { Peak Flux } \\
\left(\mathbf{k W} / \mathbf{m}^{2}\right)\end{array}$ \\
\hline 1 & $\mathrm{PE} / \mathrm{A} / \mathrm{Cal} 117$ & 64 & $50^{*}$ \\
\hline 2 & $" / \mathrm{B} / n$ & $\begin{array}{r}70 \\
(54) \\
\end{array}$ & $\begin{array}{l}17 \\
10 \\
\end{array}$ \\
\hline 3 & $" / c / n$ & 81 & 18 \\
\hline 4 & Nylon/ A / Cal 117 & 84 & $48 / 49^{*}$ \\
\hline 5 & $" / \mathrm{B} / "$ & 90 & $17 / 64^{* *}$ \\
\hline 6 & $" / \mathrm{c} /$ & $\begin{array}{l}87 \\
78 \\
\end{array}$ & $\begin{array}{l}19 / 83 \\
18 / 45 \\
\end{array}$ \\
\hline 7 & MA-N/ A /Cal 117 & 41 & $41^{*}$ \\
\hline 8 & $" / \mathrm{B} / "$ & 38 & $23^{*}$ \\
\hline 9 & $" / c / "$ & 61 & $41^{*}$ \\
\hline 10 & PE-Cot/A / Cal 117 & 87 & $80^{*}$ \\
\hline 11 & $" / B / n$ & 75 & 48 \\
\hline 12 & $" / \mathrm{c} / n$ & 72 & $17 / 49$ \\
\hline 13 & Cot/ A / Cal 117 & 76 & - \\
\hline 14 & $n /$ B / & 68 & $57^{*}$ \\
\hline 15 & $1 \mathrm{c} 1$ & 62 & $57^{*}$ \\
\hline 16 & Lt PP/ A / Cal 117 & 97 & $70^{\star}$ \\
\hline 17 & $" / \mathrm{B} / \cdots$ & 85 & $17 / 56$ \\
\hline 18 & $" / c / n$ & 67 & 65 \\
\hline 19 & Lt PP/ A /IFR & 78 & 79 \\
\hline 20 & $" / \mathrm{B} / \cdots$ & 70 & $13 / 54$ \\
\hline 21 & $" / c / \cdots$ & 80 & $25 / 54$ \\
\hline 22 & Hvy PP/A / Cal 117 & 82 & $34 / 77$ \\
\hline 23 & $" / \mathrm{B} /$ & $60 / 65$ & $11 / 25$ \\
\hline 24 & $" / \mathrm{c} /$ & $76 / 80$ & $22 / 67$ \\
\hline 25 & HVY PP/A /IFR & $\begin{array}{l}60 \\
89 \\
\end{array}$ & $\begin{array}{c}59^{\star} \\
23 / 61 \\
\end{array}$ \\
\hline 26 & $n / \mathrm{B} / n$ & $\begin{array}{l}75 \\
68 \\
\end{array}$ & $\begin{array}{c}12 \\
9 / 14 \\
\end{array}$ \\
\hline 27 & $" / c / *$ & 85 & $14 / 64$ \\
\hline
\end{tabular}

"Both gages in corner region, ca. $2 \mathrm{~cm}$ apart.

"*here two values are shown, the first is during the burner exposure and the second occurs later, but during first RHR peak. 
Table 6

Estimate of Effective Peak External Flux

During Gas Burner Exposure in Mock-Ups

\begin{tabular}{|c|c|c|c|}
\hline Materials & $\begin{array}{l}\text { Flame Flux } \\
\text { In Cone } \\
\left(\mathrm{kW} / \mathrm{m}^{2}\right)\end{array}$ & $\begin{array}{c}\text { Gage Flux in } \\
\text { Mock-Up }\left(\mathrm{kW} / \mathrm{m}^{2}\right)\end{array}$ & $\begin{array}{c}\text { Effective } \\
\text { External Flux } \\
\text { in Full-Scale } \\
\left(\mathrm{kW} / \mathrm{m}^{2}\right)\end{array}$ \\
\hline $\mathrm{MA}-\mathrm{N} / \mathrm{C} / \mathrm{Cal} 117^{*}$ & $22-26$ & 61 & $35-39$ \\
\hline Cot/ A / Cal 117 & $15-18$ & 76 & $58-61$ \\
\hline Hvy PP/ A / Cal 117 & $25-28$ & 82 & $54-57$ \\
\hline
\end{tabular}

* Full-scale data from the test with Barrier $C$ are used rather than with Barrier A since the former was a better-behaved mock-up test and should be more properly representative of the behavior with this fabric. 
ALL CUSHIONS HAVE WELT CORDS
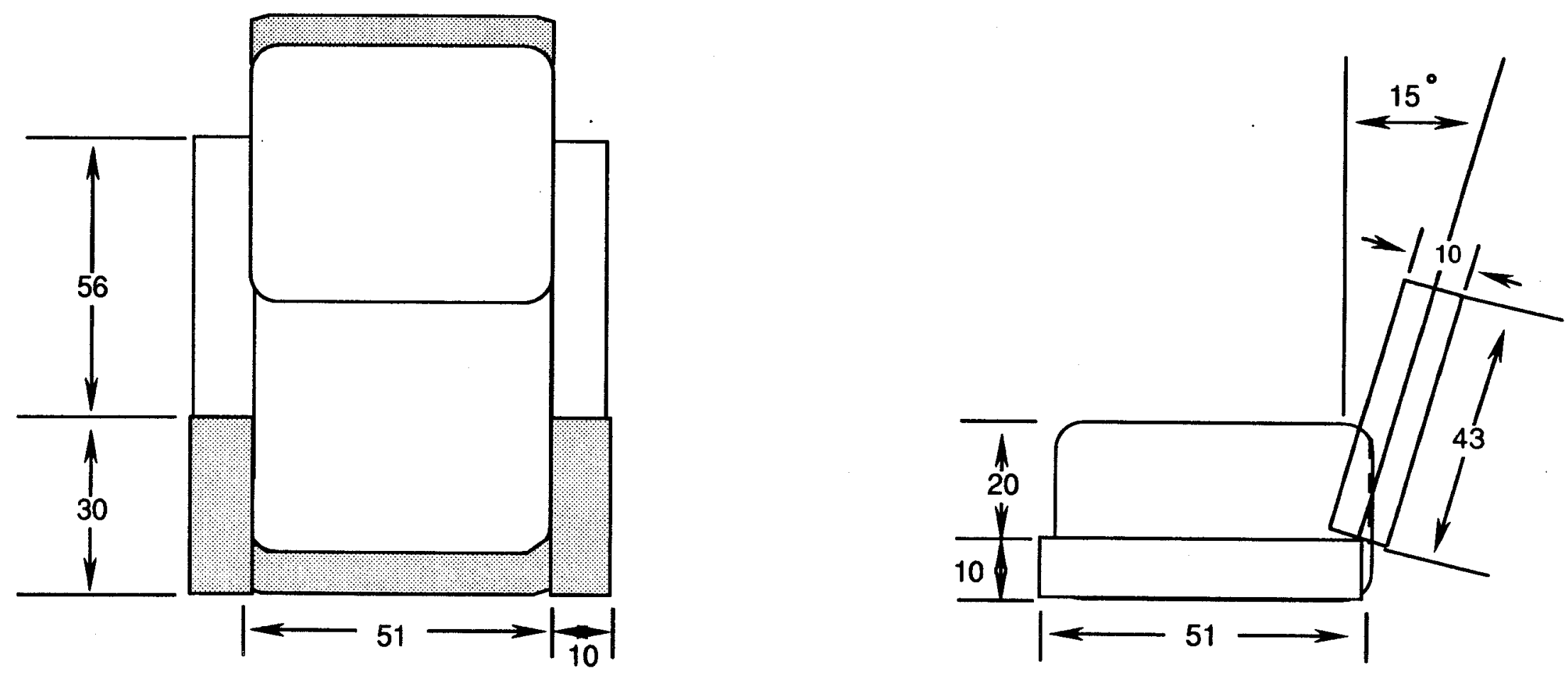

ALL DIMENSIONS IN CENTIMETERS

FIGURE 1 CUSHION ARRANGEMENT IN MOCK-UPS 


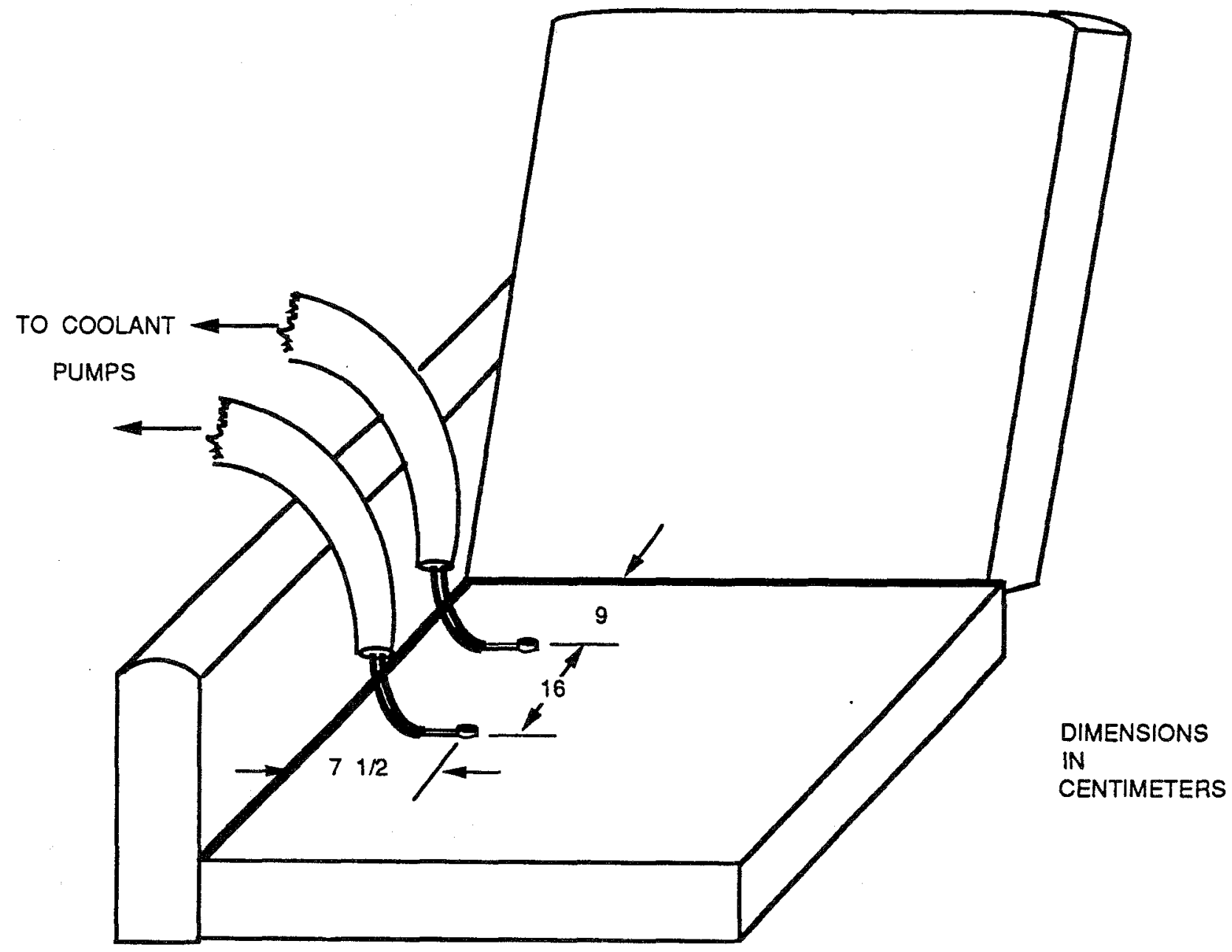

FIG. 2 APPROXIMATE PLACEMENT OF HEAT

FLUX GAGES ON MOCK - UP SEAT

CUSHION 

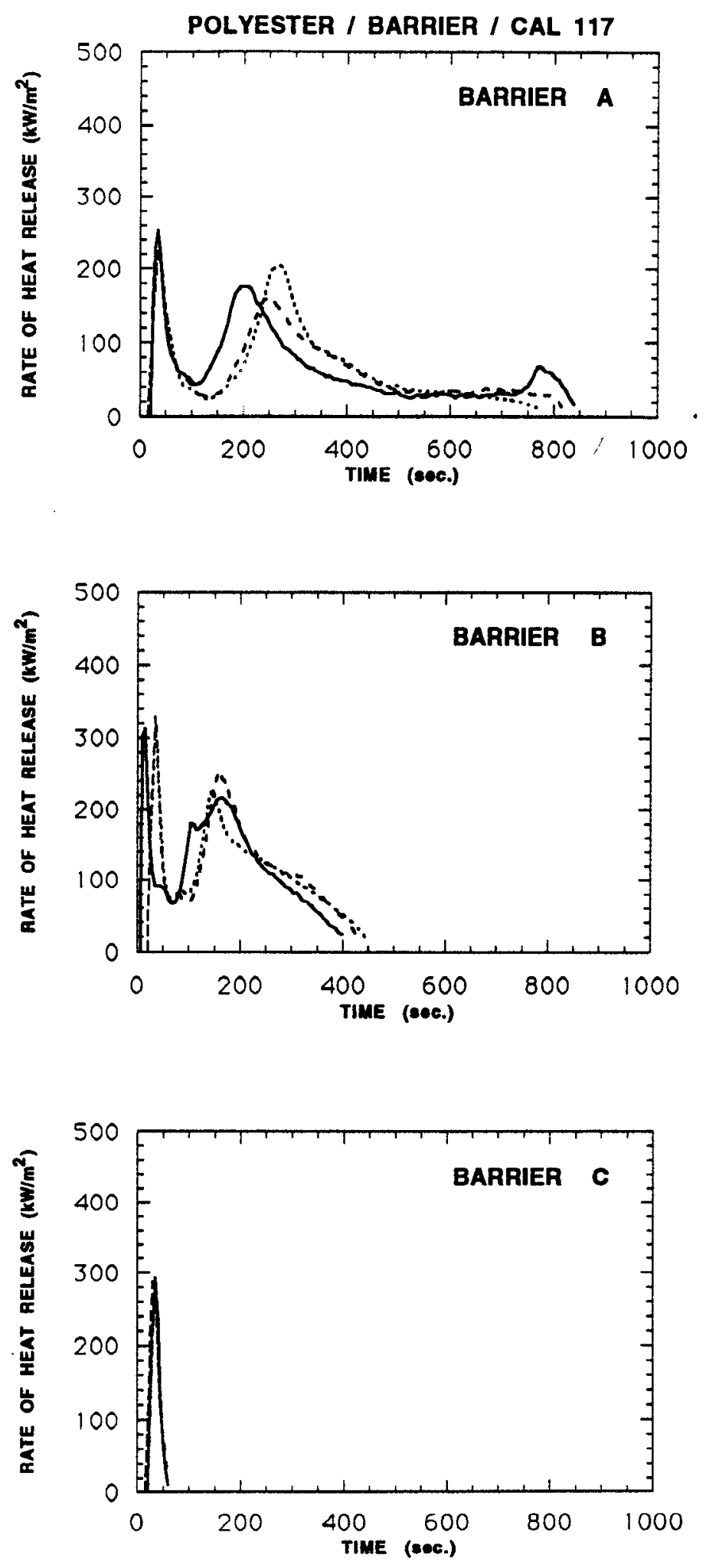

FIG. 3 CONE CALORIMETER DATA AT $35 \mathrm{~kW} / \mathrm{m}^{2}$ POLYESTER / BARRIER / CAL 117 

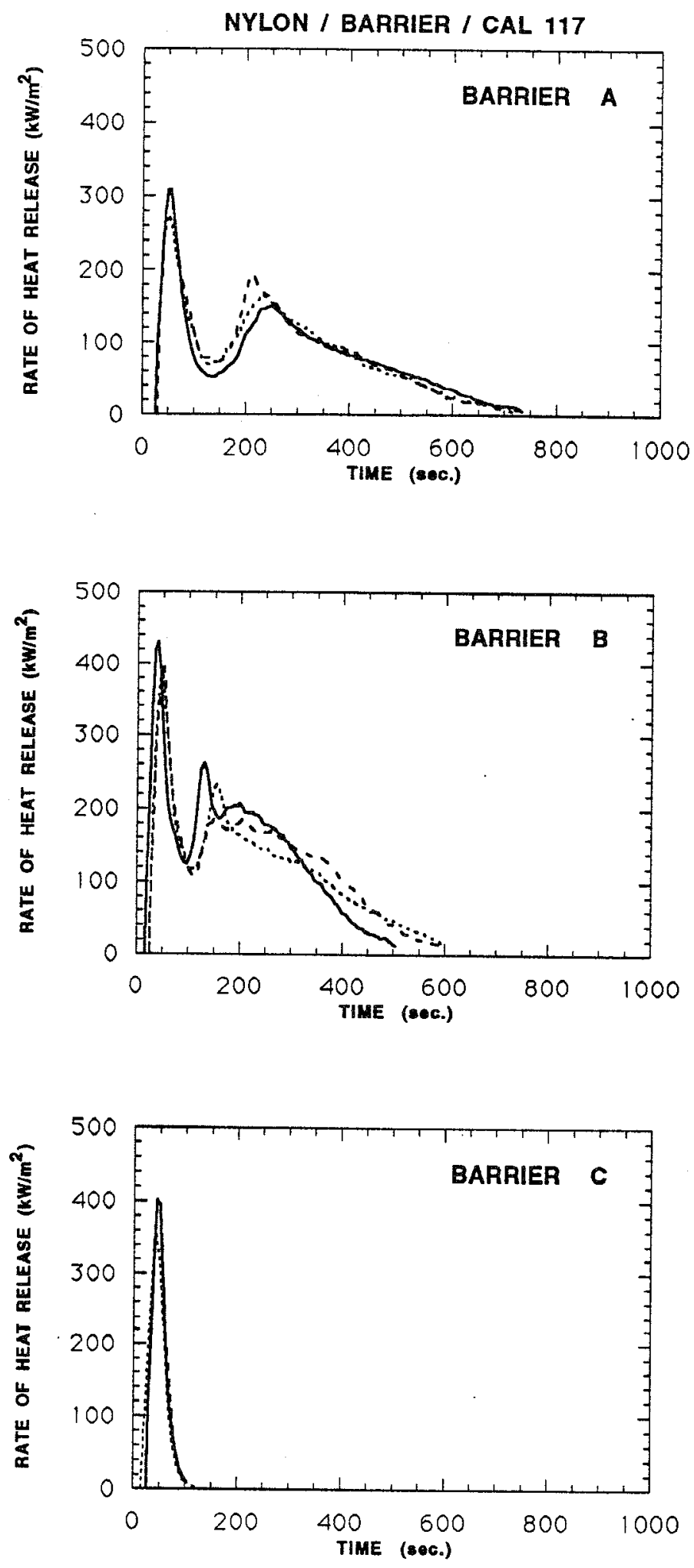

FIG. 4 CONE CALORIMETER DATA AT $35 \mathrm{~kW} / \mathrm{m}^{2}$ NYLON / BARRIER/CAL 117 

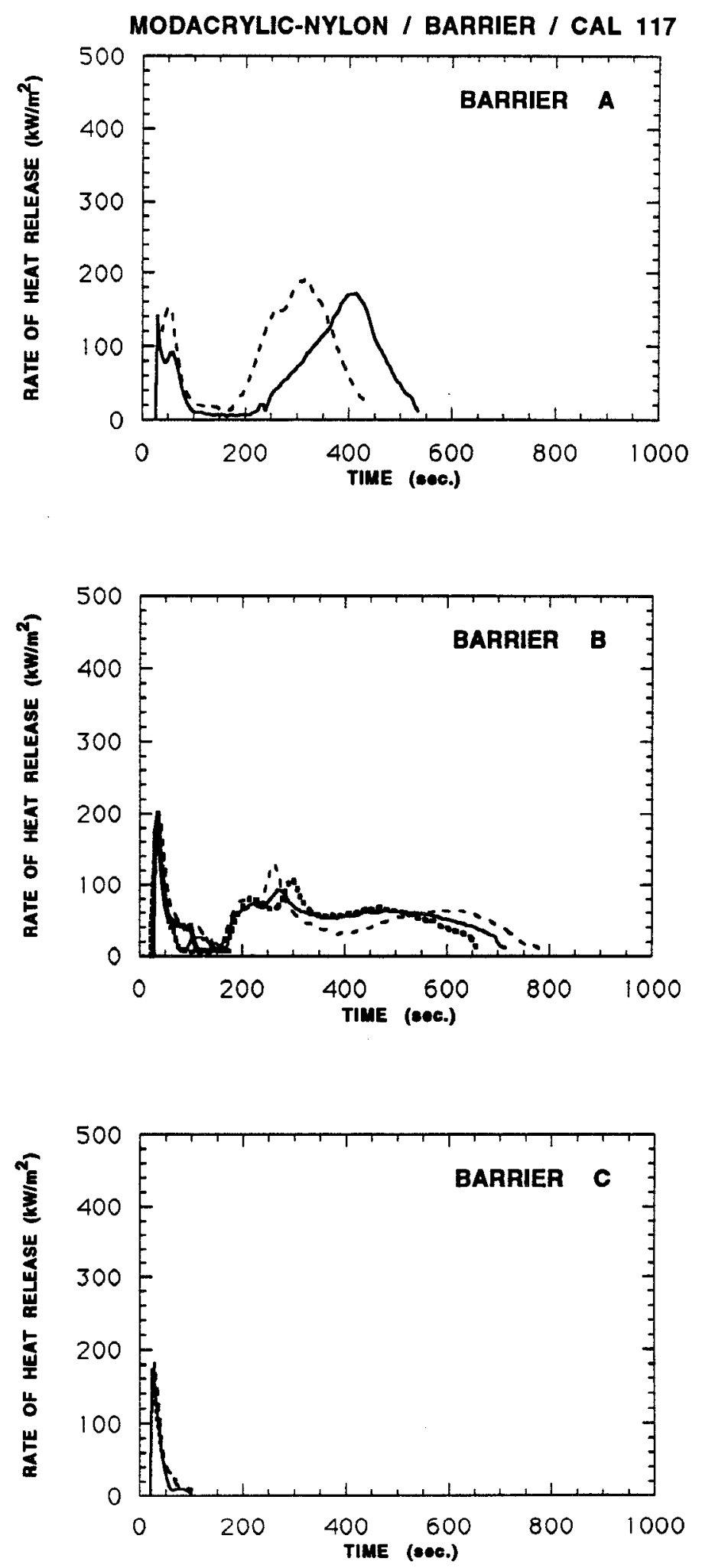

FIG. 5 CONE CALORIMETER DATA AT $35 \mathrm{~kW} / \mathrm{m}^{2}$ MODACRYLIC-NYLON / BARRIER / CAL 117

39 

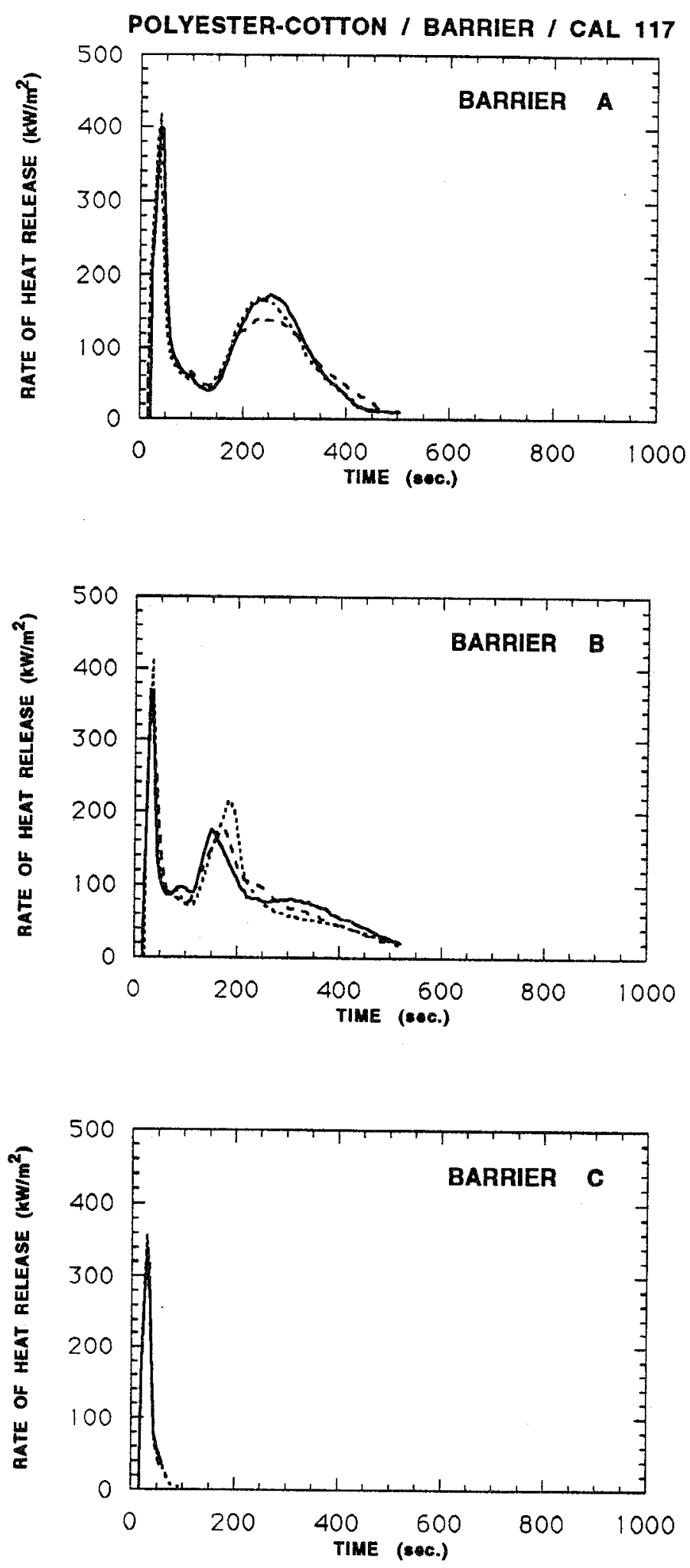

FIG. 6 CONE CALORIMETER DATA AT $35 \mathrm{~kW} / \mathrm{m}^{2}$ POLYESTER-COTTON / BARRIER / CAL 117 

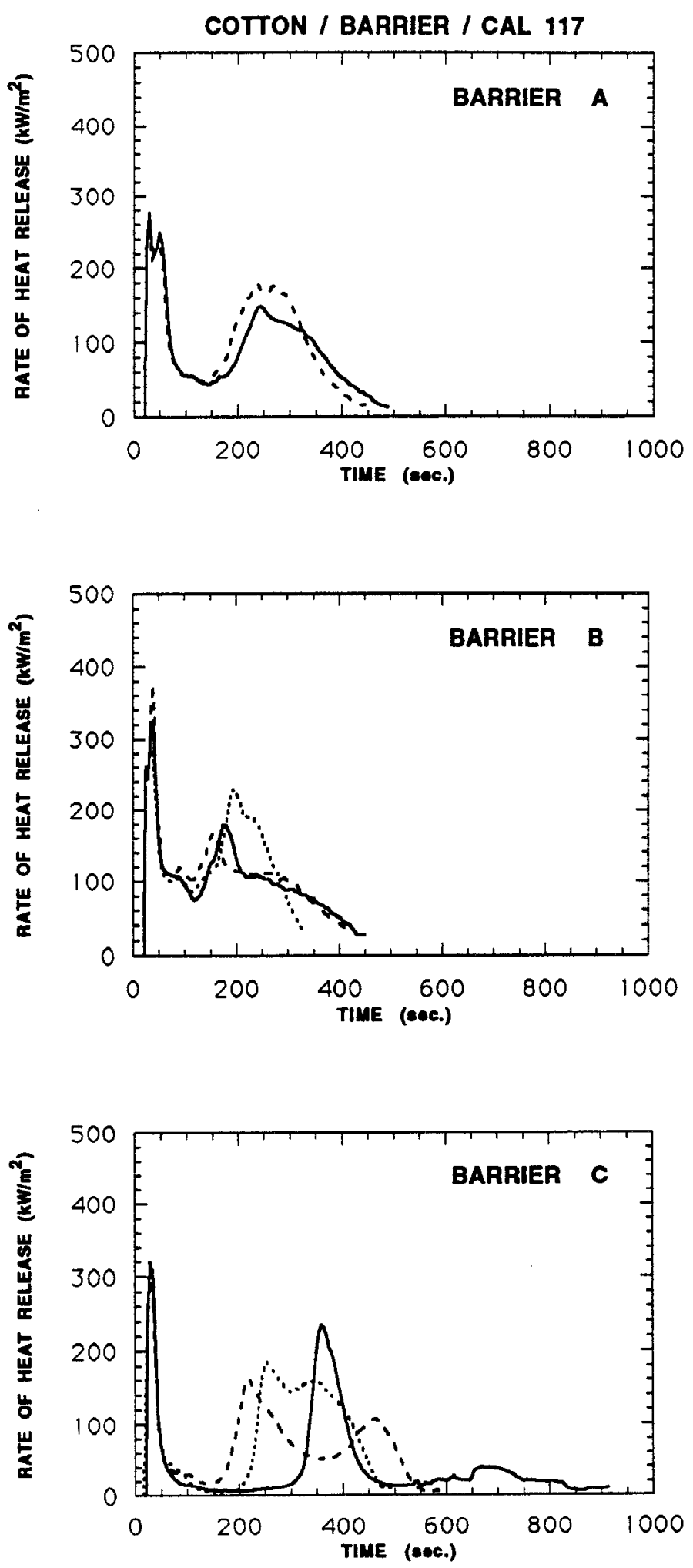

FIG. 7 CONE CALORIMETER DATA AT $35 \mathrm{~kW} / \mathrm{m}^{2}$ COTTON/BARRIER / CAL 117 
LIGHT POLYPROPYLENE / BARRIER / CAL 117
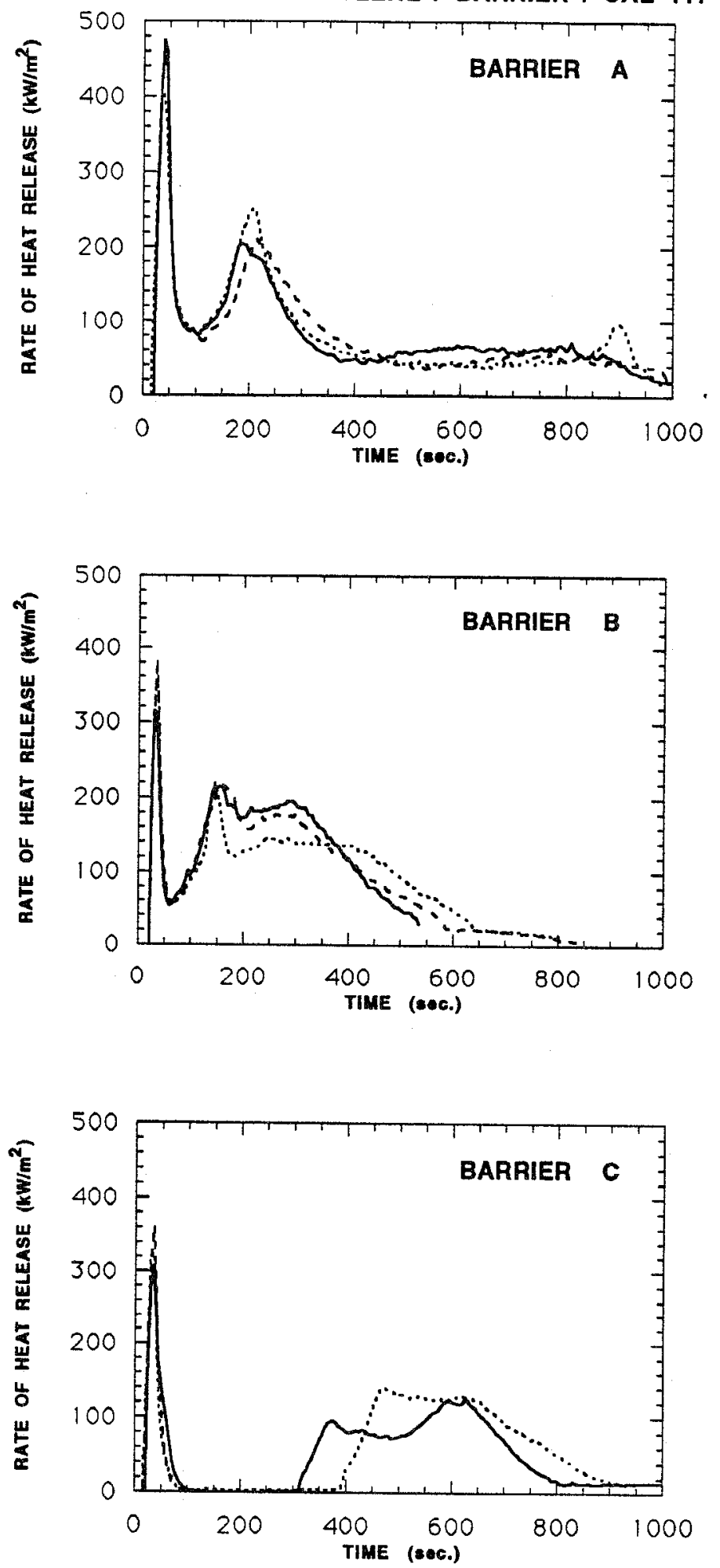

FIG. 8 CONE CALORIMETER DATA AT $35 \mathrm{~kW} / \mathrm{m}^{2}$ LIGHT POLYPROPYLENE / BARRIER / CAL 117 

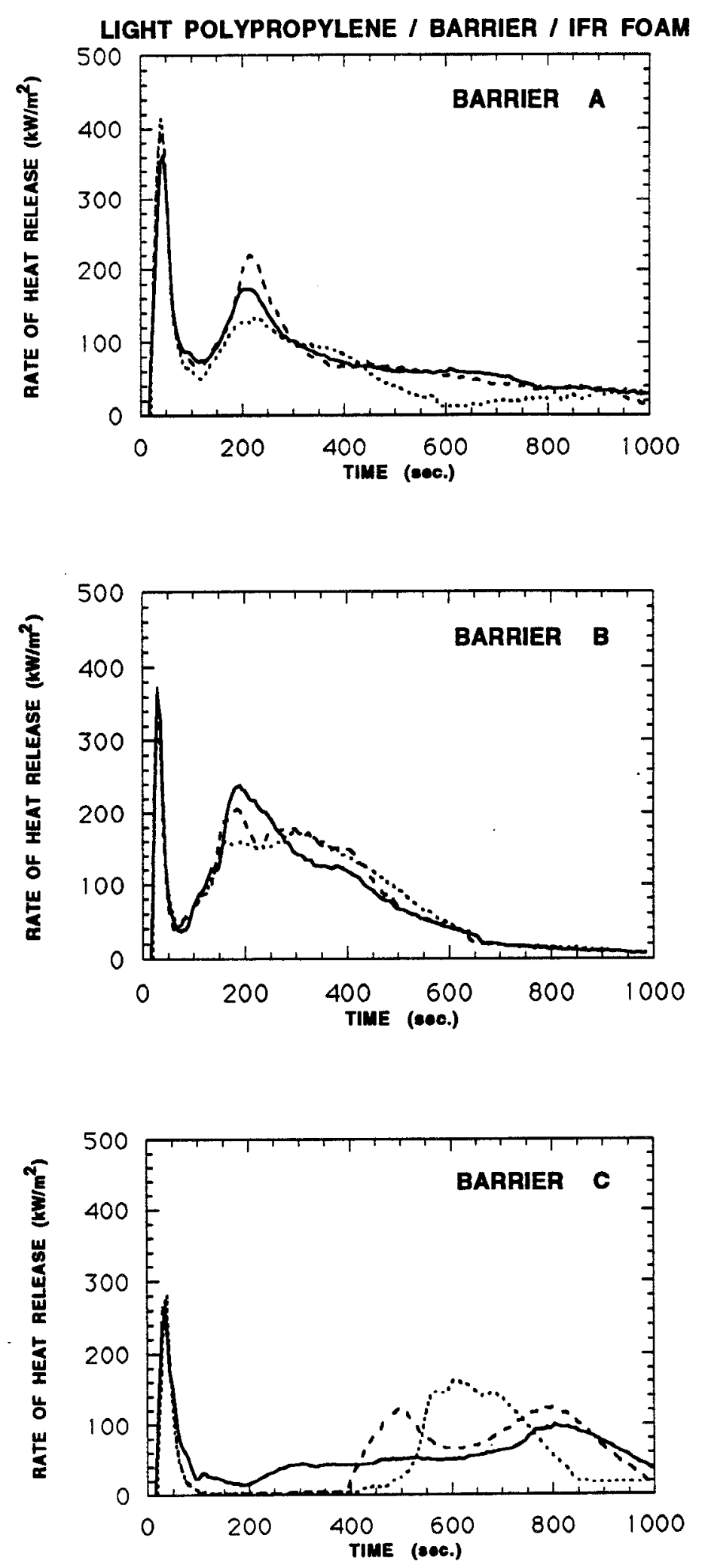

FIG. 9 CONE CALORIMETER DATA AT $35 \mathrm{~kW} / \mathrm{m}^{2}$ LIGHT POLYPROPYLENE / BARRIER / IFR FOAM 

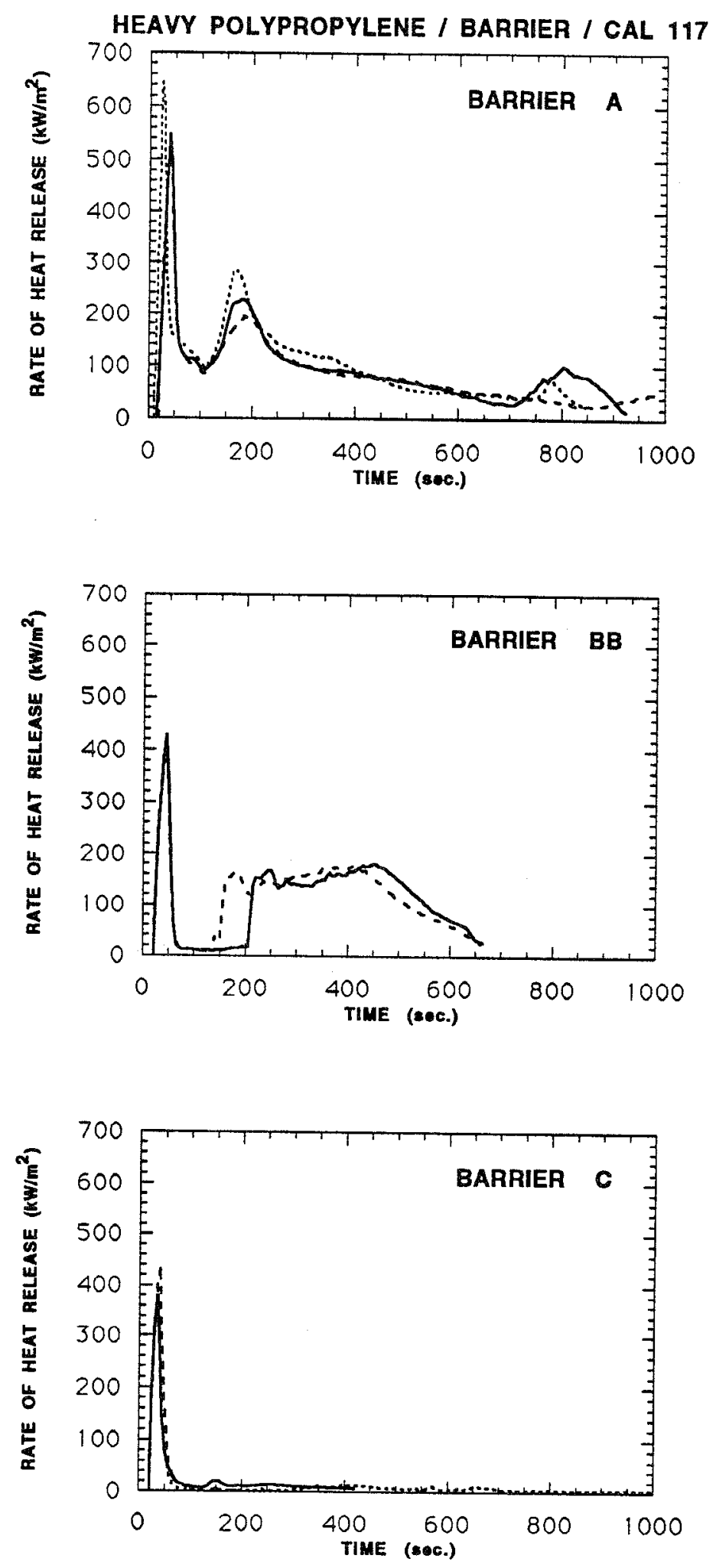

FIG. 10 CONE CALORIMETER DATA AT $35 \mathrm{~kW} / \mathrm{m}^{2}$

HEAVY POLYPROPYLENE / BARRIER / CAL 117 

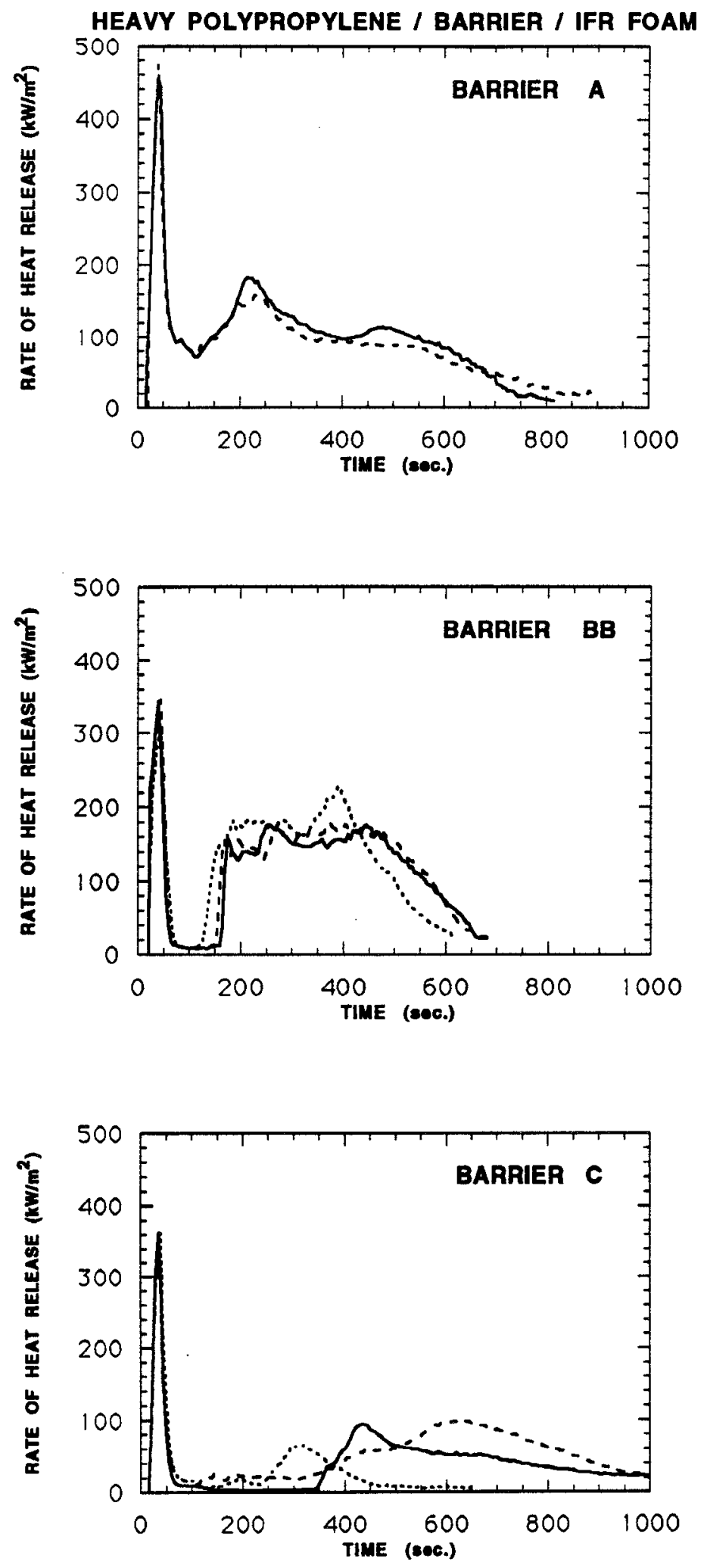

FIG. 11 CONE CALORIMETER DATA AT $35 \mathrm{~kW} / \mathrm{m}^{2}$ HEAVY POLYPROPYLENE / BARRIER / FR FOAM

45 

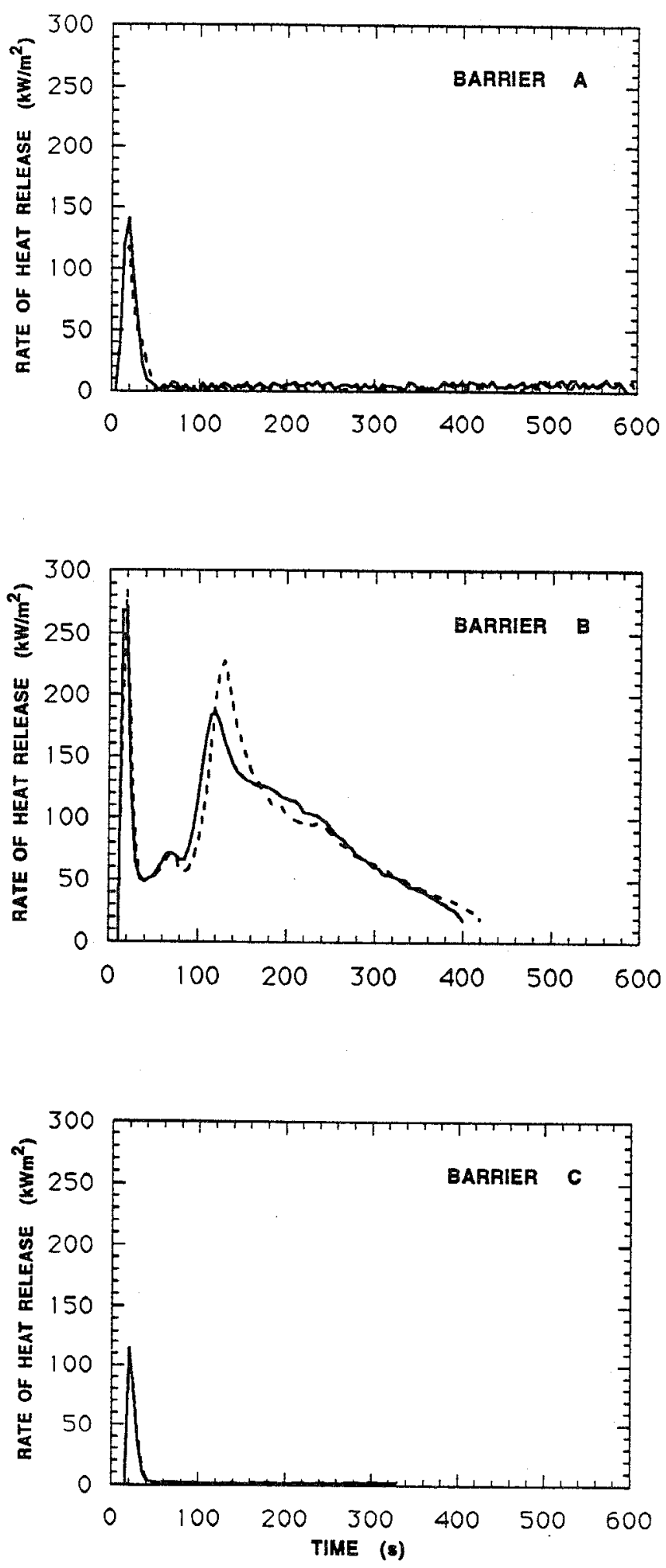

FIG. 12 RATE OF HEAT RELEASE FOR BARRIER ALONE ATOP CAL 117 FOAM AT $35 \mathrm{kw} / \mathrm{m}^{2}$ 
FIGURE 13

FLUX DEPENDENCE OF HEAT RELEASE RATE

ALL FABRICS ON BARRIER AV CAL 117

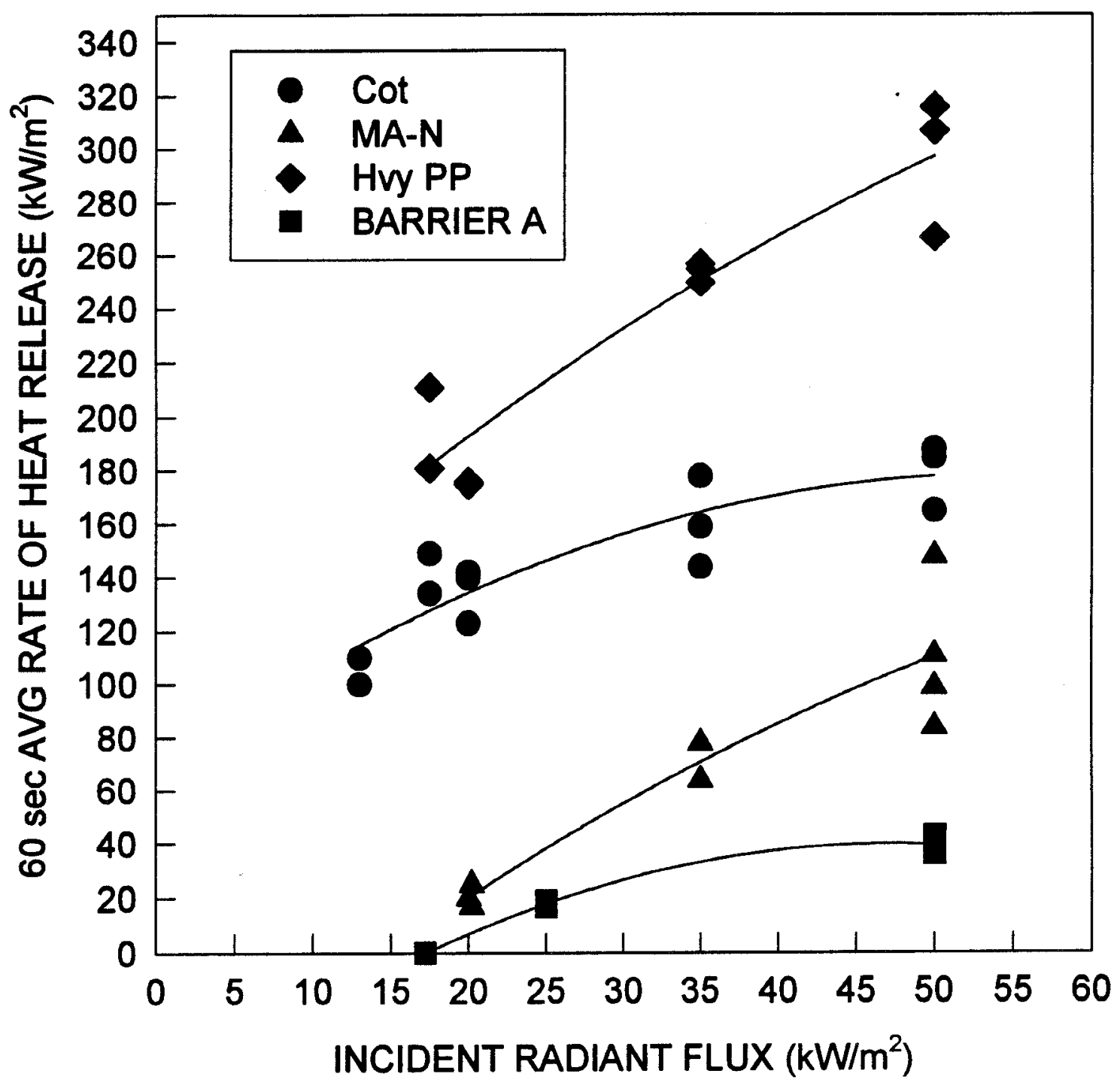




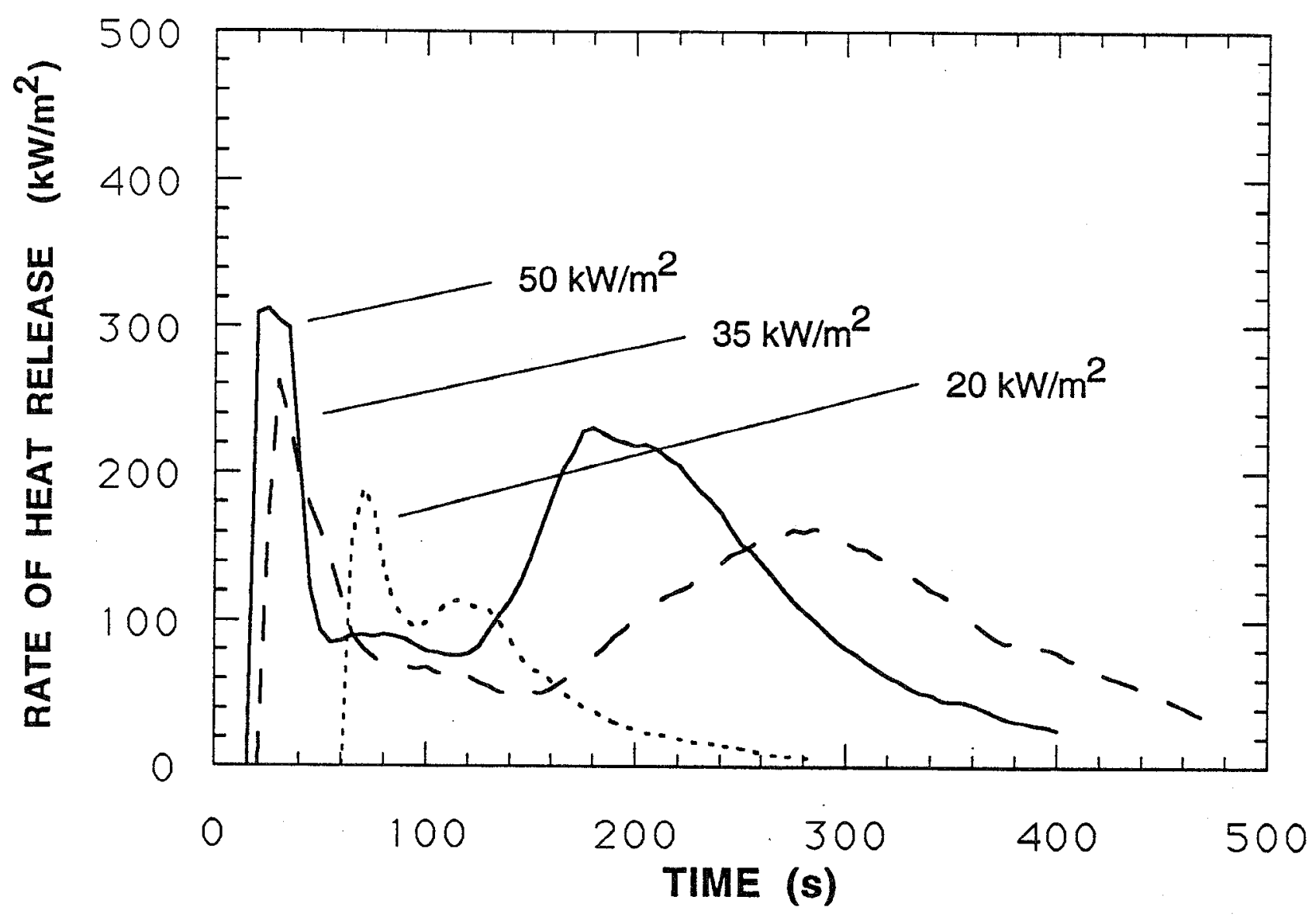

FIG. 14 RATE OF HEAT RELEASE AS A FUNCTION OF INCIDENT RADIANT FLUX FOR

COTTON / BARRIER A / CAL 117 

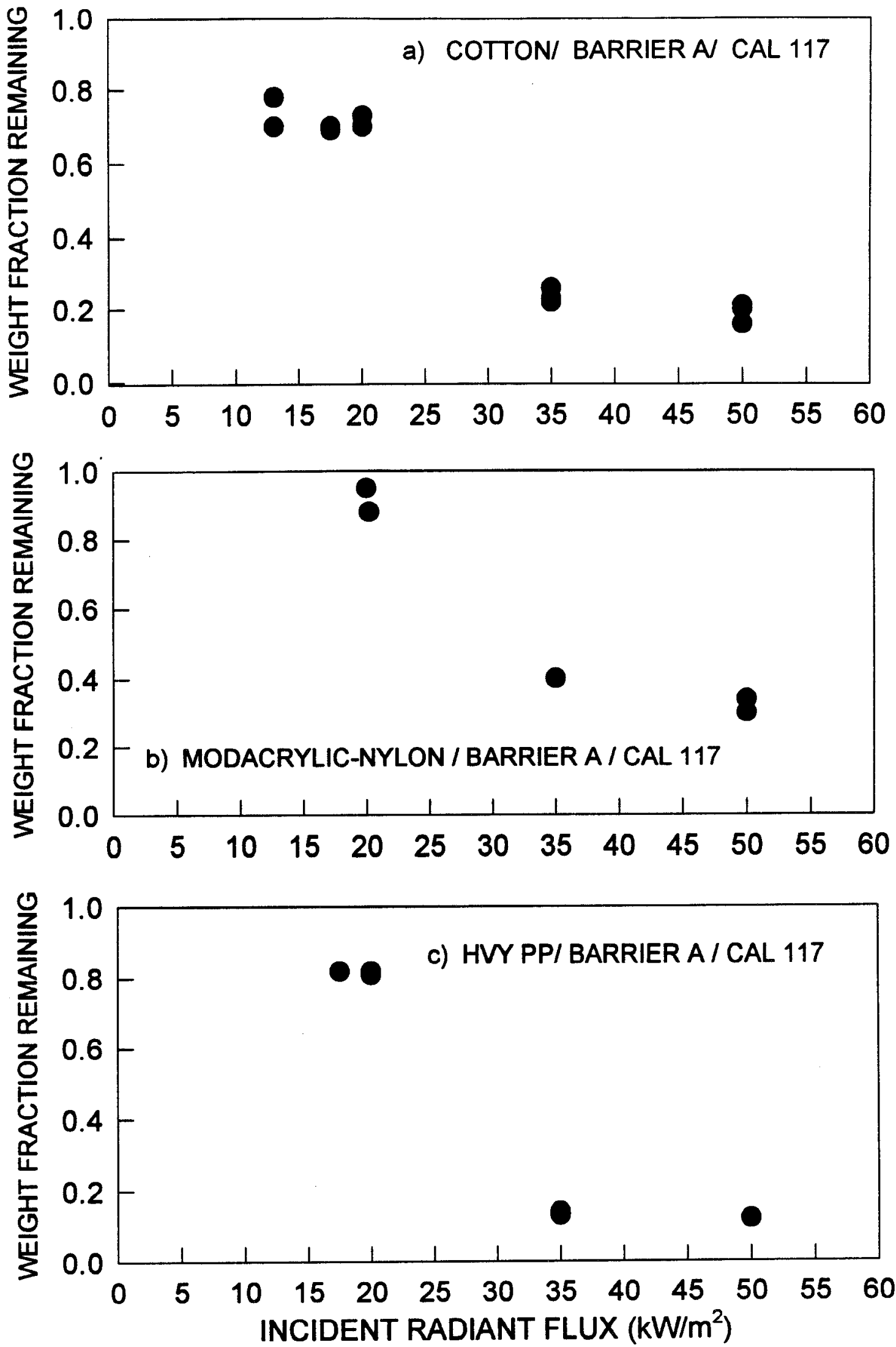

FIGURE 15. WEIGHT FRACTION REMAINING AFTER CONE CALORIMETER TEST 

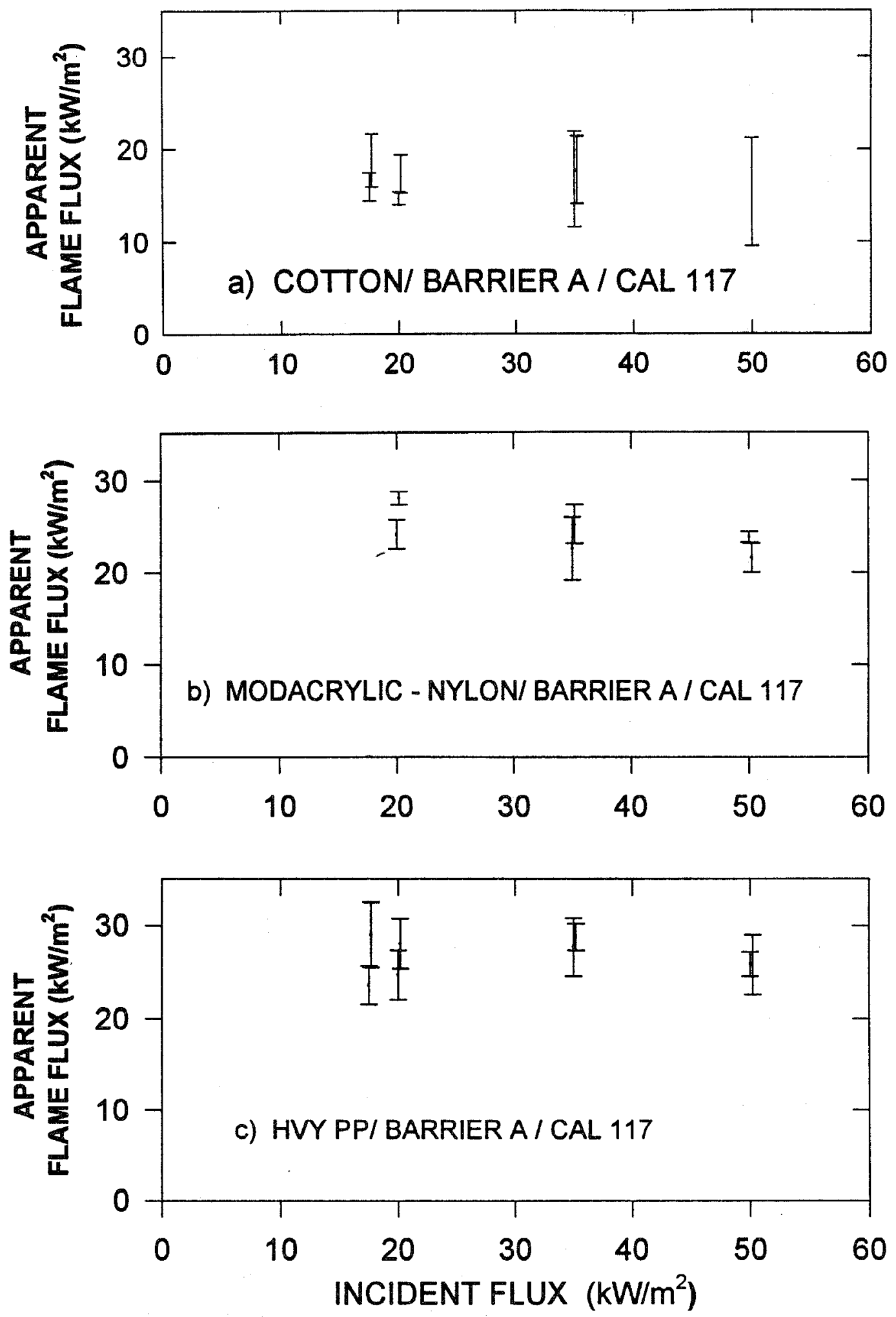

FIGURE 16 FLAME HEAT FLUX TO GAGE SURFACE IN CONE CALORIMETER 

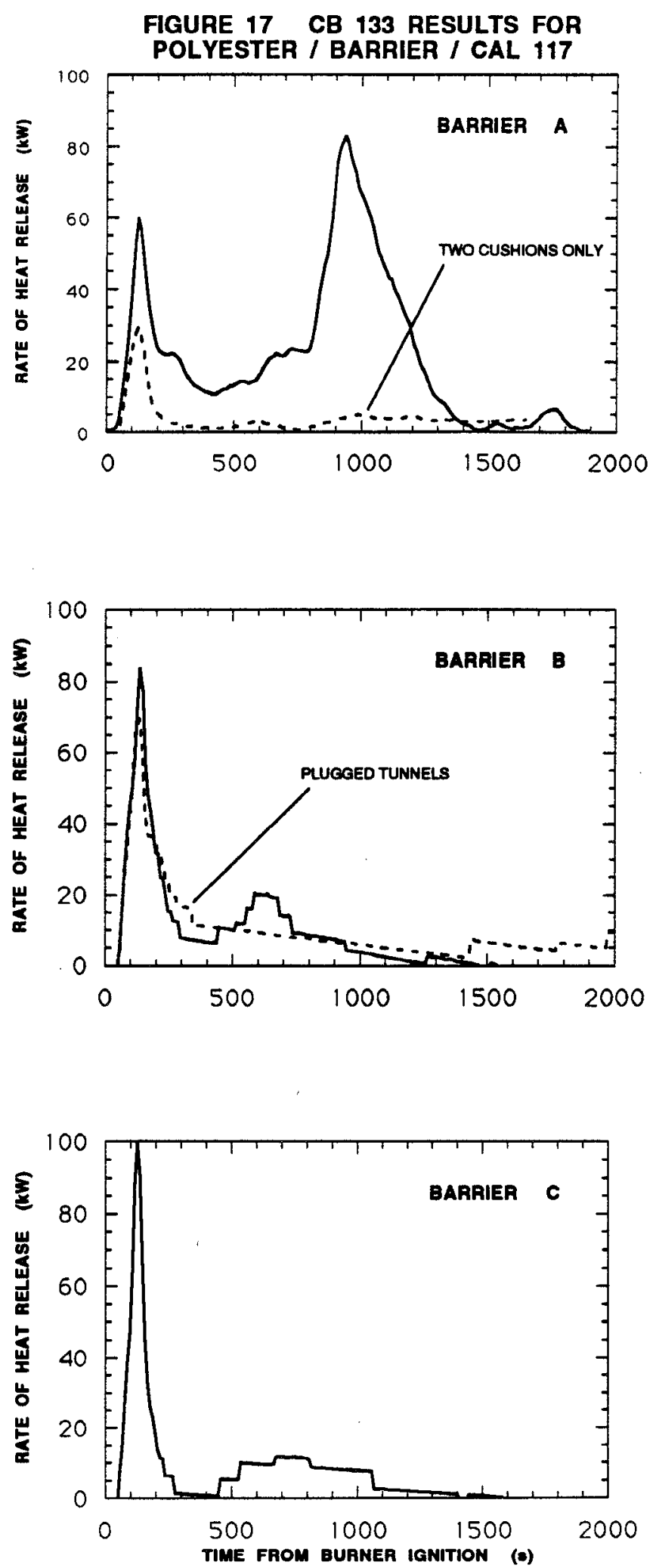
FIGURE 18 CB 133 RESULTS FOR

NYLON / BARRIER / CAL 117

BARRIER A

RHR SYSTEM FAILURE (VISUAL ESTIMATES ONLY) FIRST PEAK $=100 \mathrm{~kW}$

SECOND PEAK $300 \mathrm{~kW}$
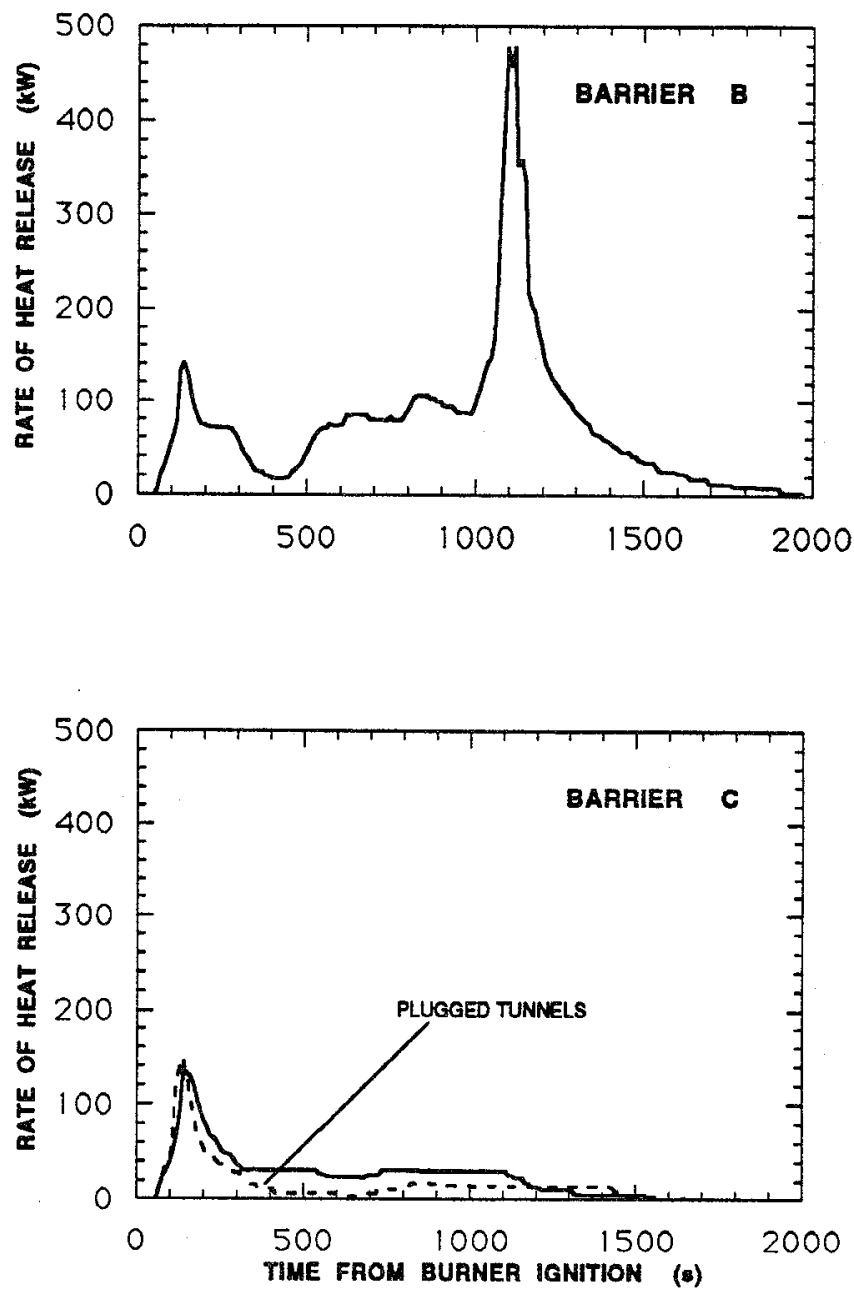
FIGURE 19 CB 133 RESULTS FOR

MODACRYLIC-NYLON / BARRIER / CAL 117

\section{BARRIER A}

RHR SYSTEM FAILURE (VISUAL ESTIMATES ONLY) FIRST PEAK $=35 \mathrm{~kW}$ NO SECOND PEAK
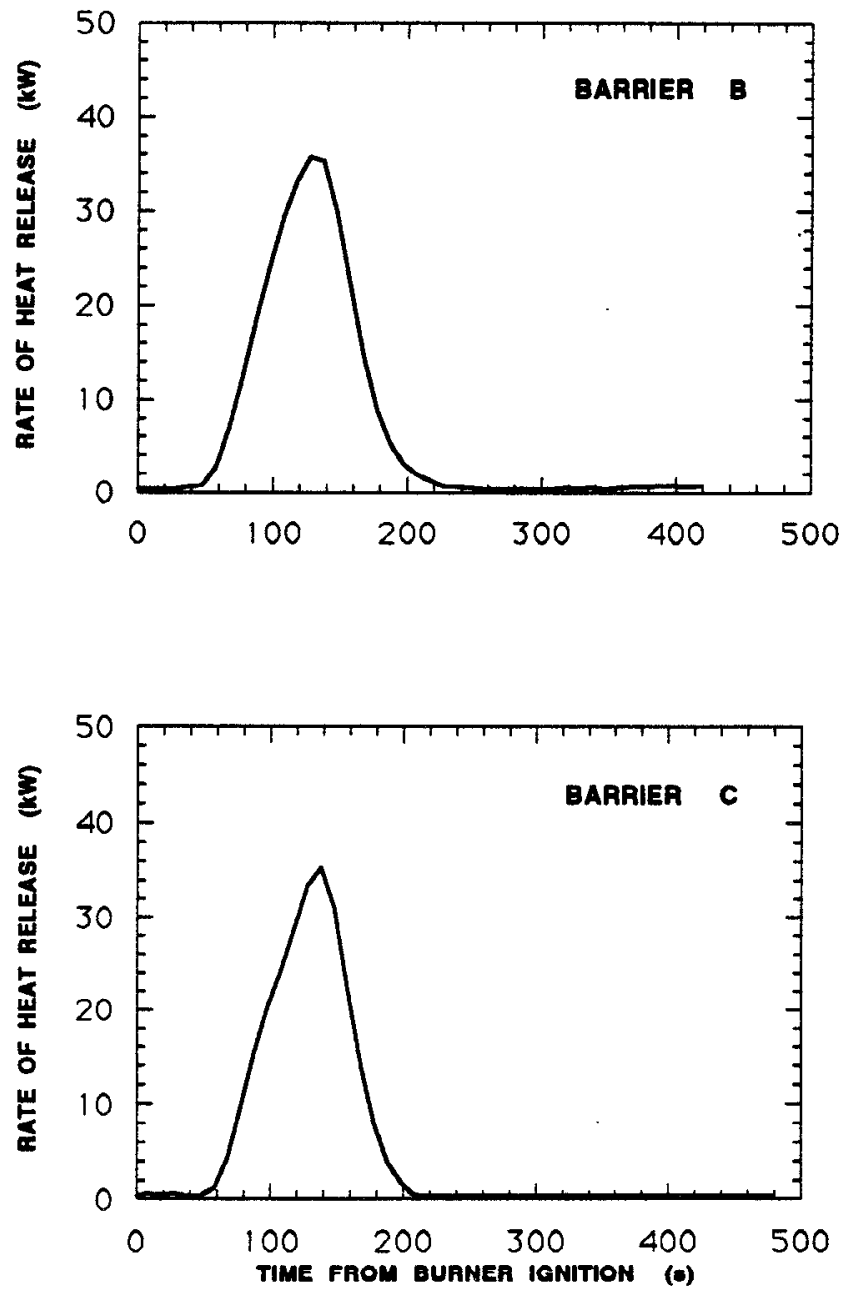
FIGURE 20 CB 133 RESULTS FOR
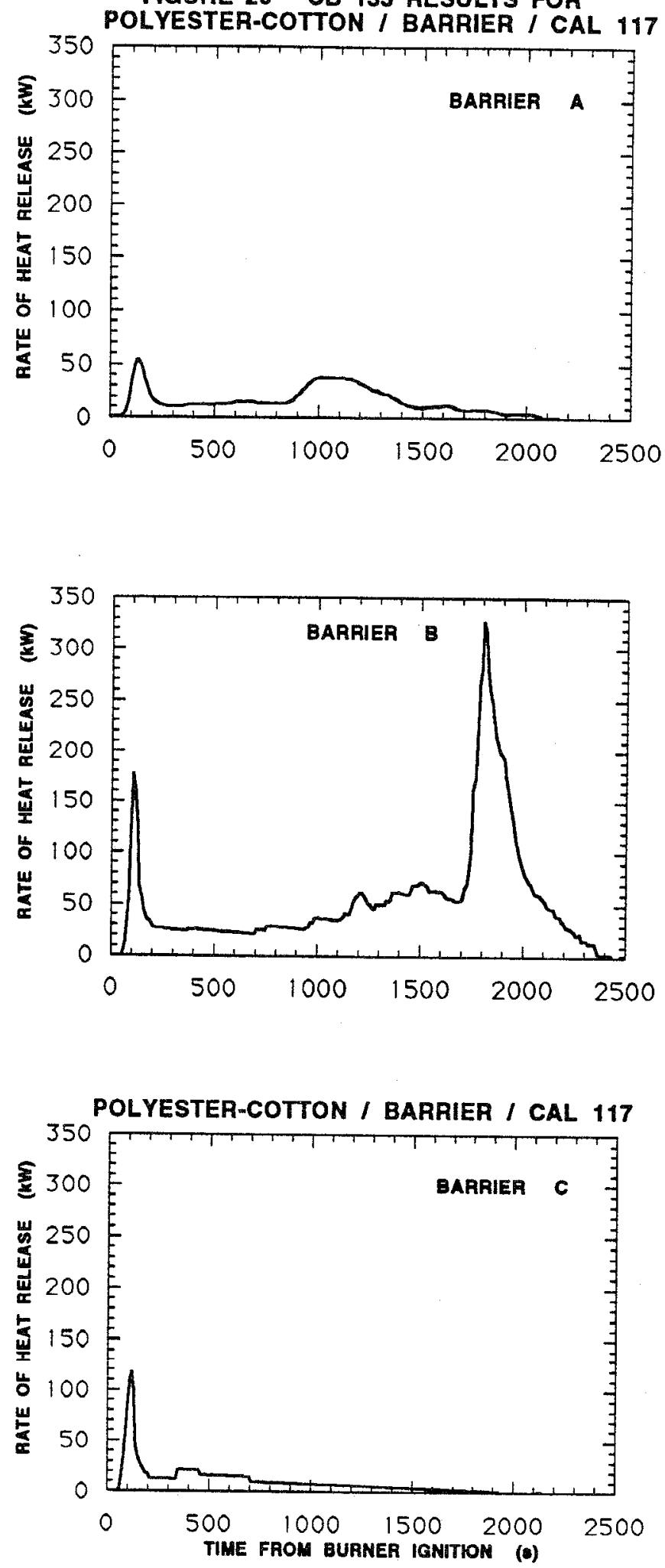

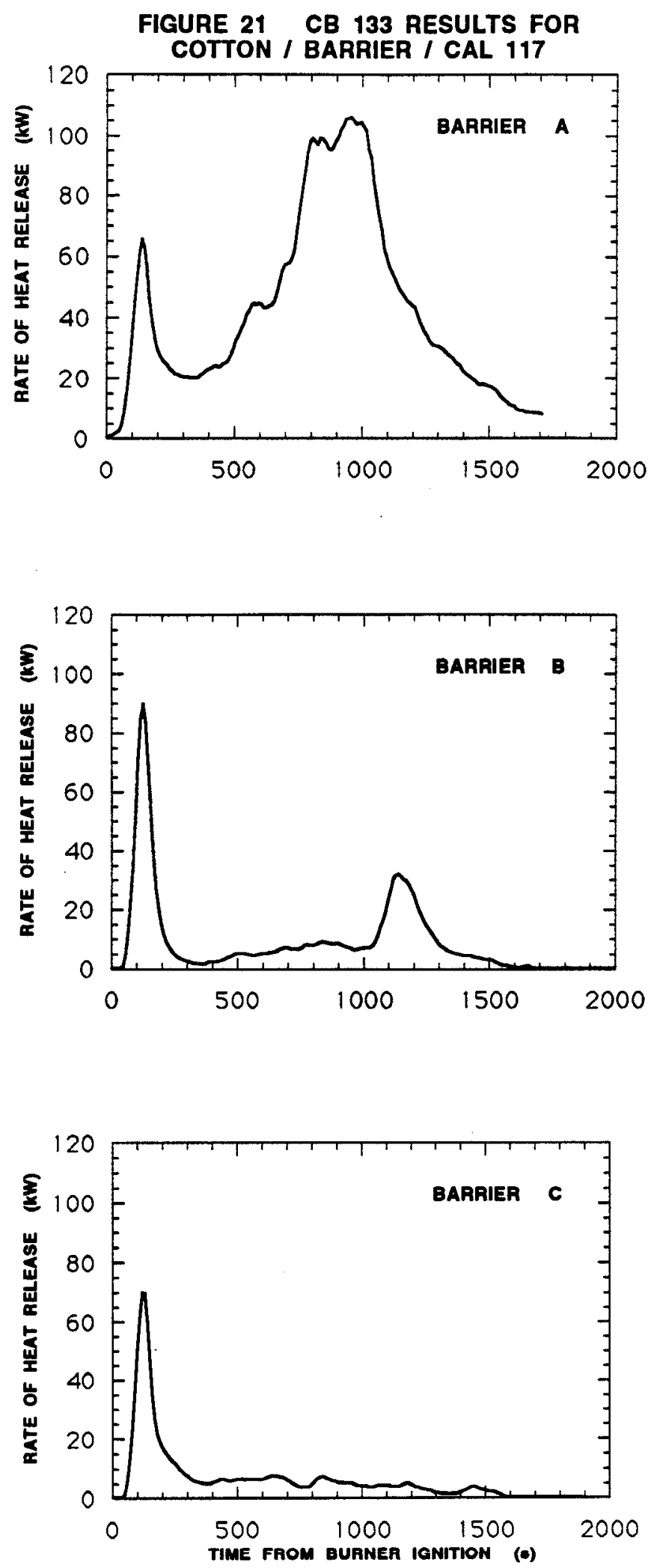
FIGURE 22 CB 133 RESULTS FOR

LIGHT POLYPROPYLENE / BARRIER / CAL 117
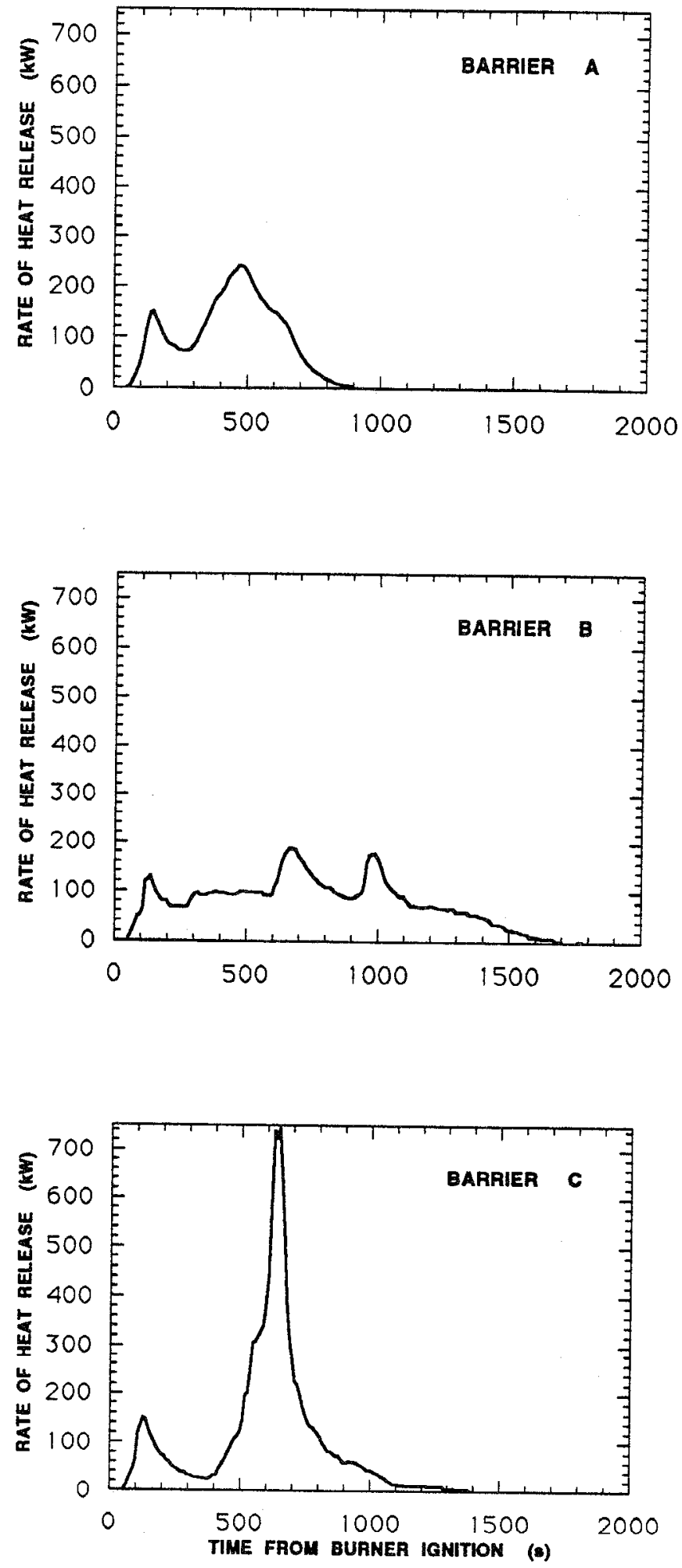
FIGUAE 23 CB 133 RESULTS FOR

LIGHT POLYPROPYLENE / BARRIER / IFR FOAM
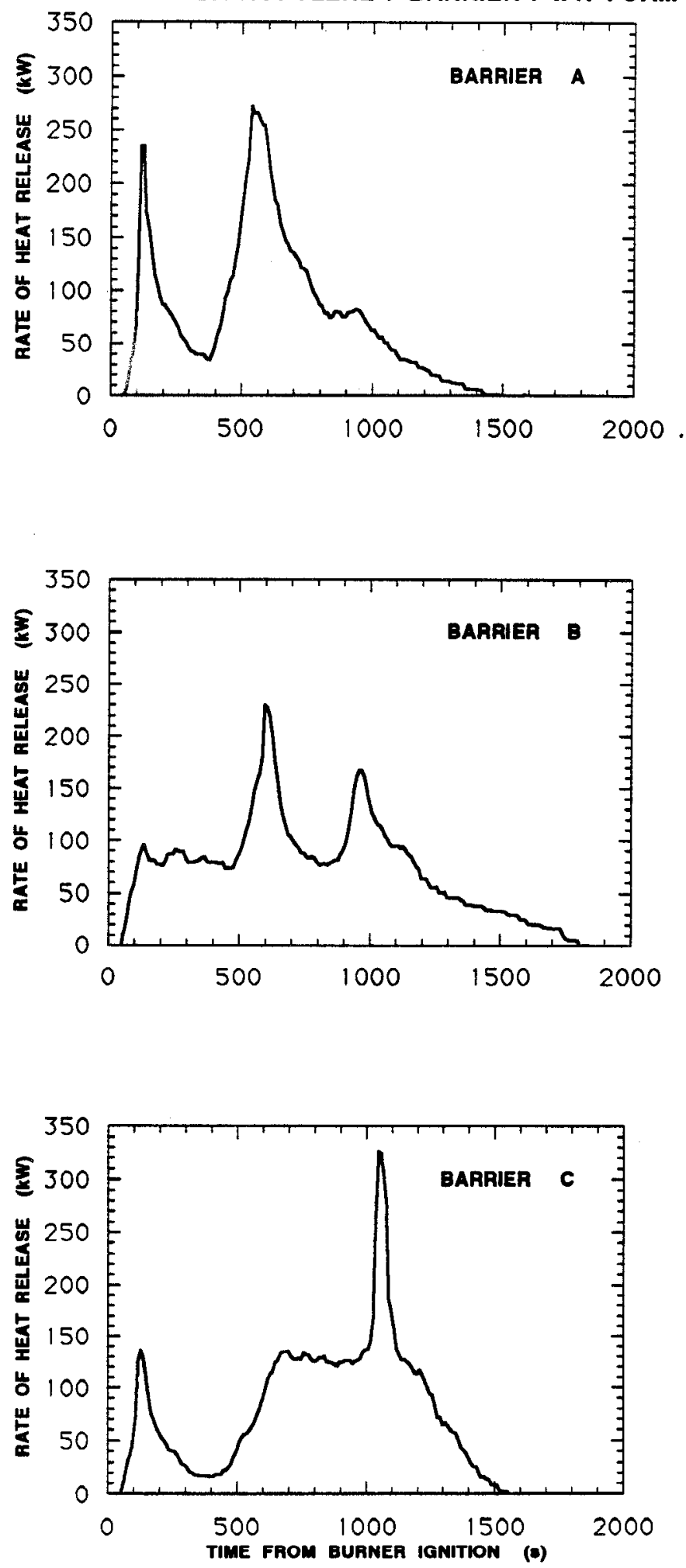
FIGURE 24 CB 133 RESULTS FOR

HEAVY POLYPROPYLENE / BARRIER / CAL 117
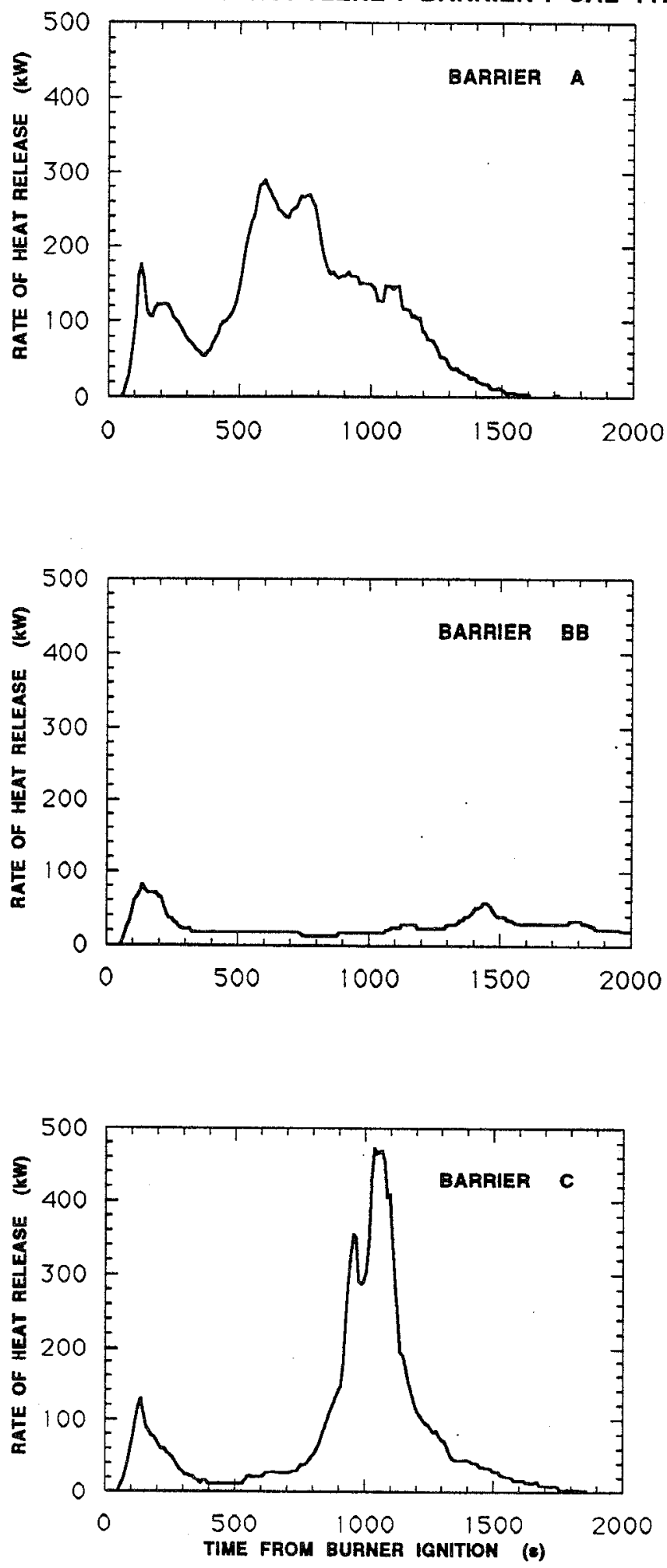
FIGURE 25 CB 133 RESULTS FOR
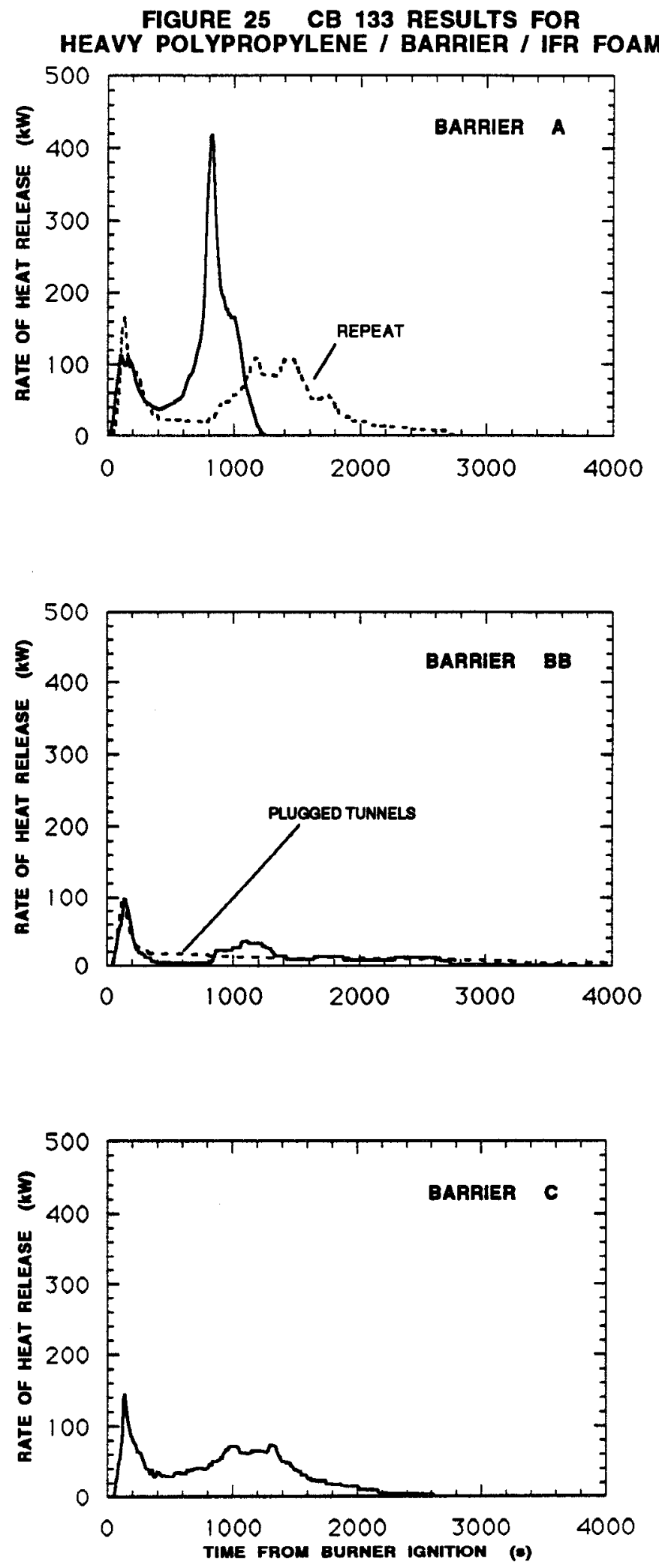
FIGURE 26a

\section{PEAK HEAT RELEASE RATE DURING BURNER EXPOSURE}

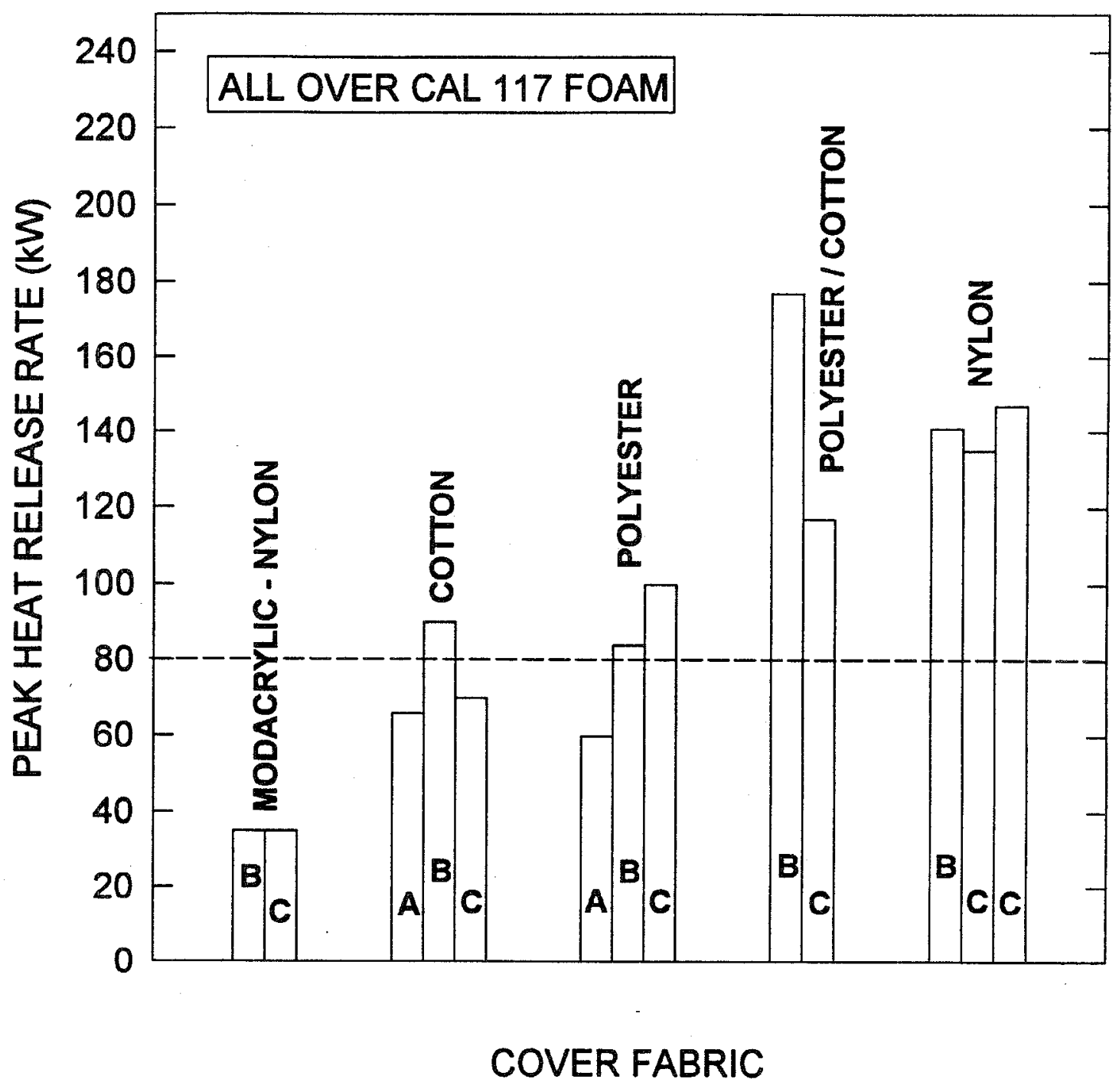


FIGURE $26 \mathrm{~b}$

\section{PEAK HEAT RELEASE RATE DURING BURNER EXPOSURE (100\% POLYPROPYLENE FABRICS)}

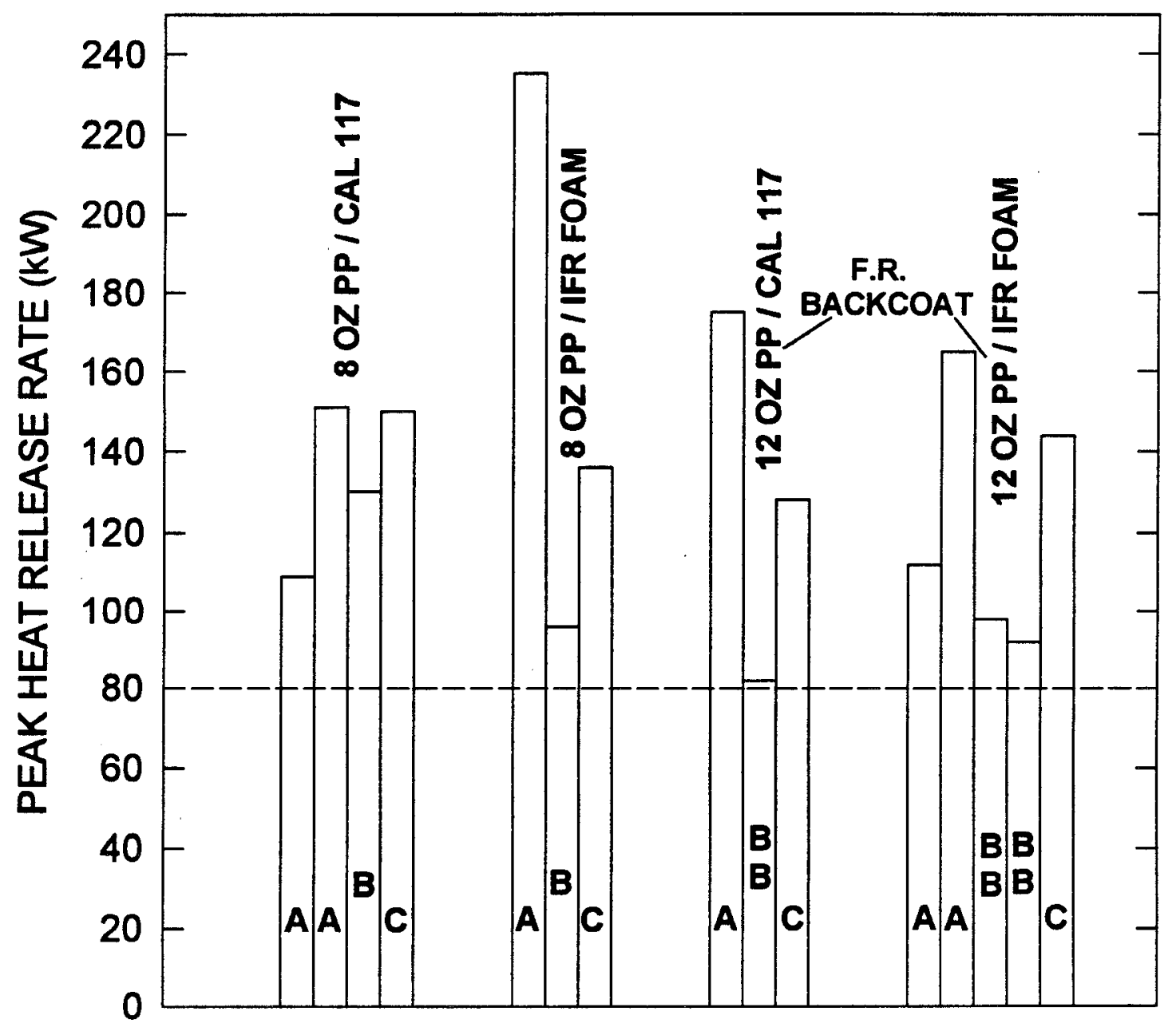

COVER FABRIC AND FOAM TYPE 


\section{FIRE GROWTH HISTORY DURING BURNER EXPOSURE}

MOCK-UP: NYLON / BARRIER B / CAL 117 FOAM

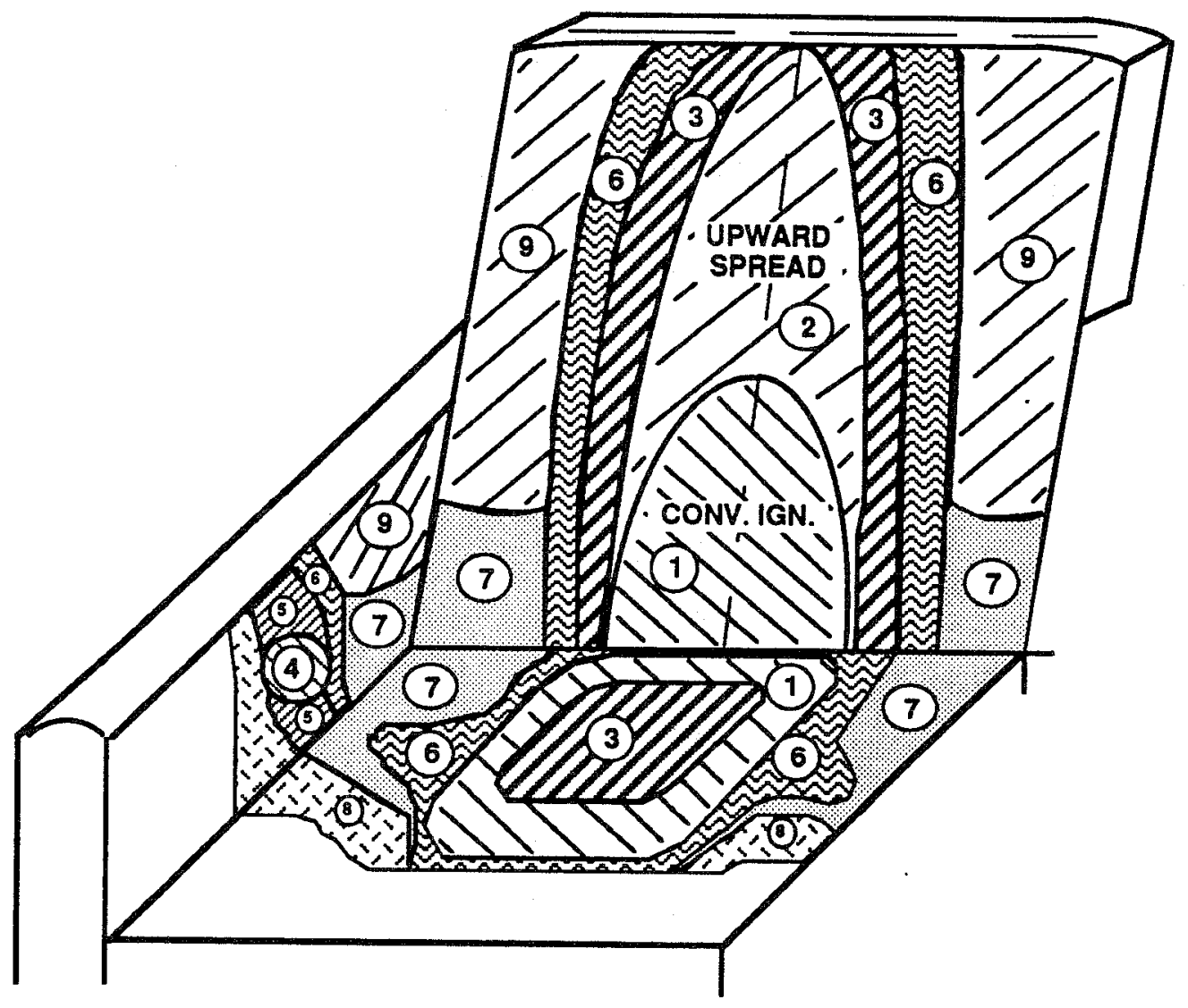

1) CONVECTIVE IGNITION ON SEATBACK (15 s) \& SEAT (27 s)

2) UPWARD FLAME SPREAD ON SEATBACK (15 - $27 \mathrm{~s})$

3) ANGLED UPWARD SPREAD ON SEATBACK (27 - 40 s) AND OPPOSED FLOW SPREAD ON SEAT (27 - $40 \mathrm{~s})$

4) CONVECTIVE / RADIATIVE IGNITION OF INNER ARMS (40 s)

5) UPWARD FLAME SPREAD ON INNER ARMS (40 - $51 \mathrm{~s}$ )

6) COMPOUND FLAME SPREAD (40 - $60 \mathrm{~s}$ )

(CURLBACK OF FABRIC + SPREAD ON EXPOSED BARRIER SURFACE)

7) RADIATIVE IGNITION (66 - $70 \mathrm{~s}$ )

8) OPPOSED FLOW SPREAD TOWARD FRONT OF CHAIR (70-80 s)

9) UPWARD FLAME SPREAD ON INNER ARMS (70 - $75 \mathrm{~s})$ AND OUTER AREAS OF SEATBACK $(70-80 \mathrm{~s})$ 
FIGURE 28

\section{FIRE SPREAD DURING BURNER EXPOSURE}

- AREA AFFECTED BY FIRE

- AREA CURRENTLY FLAMING
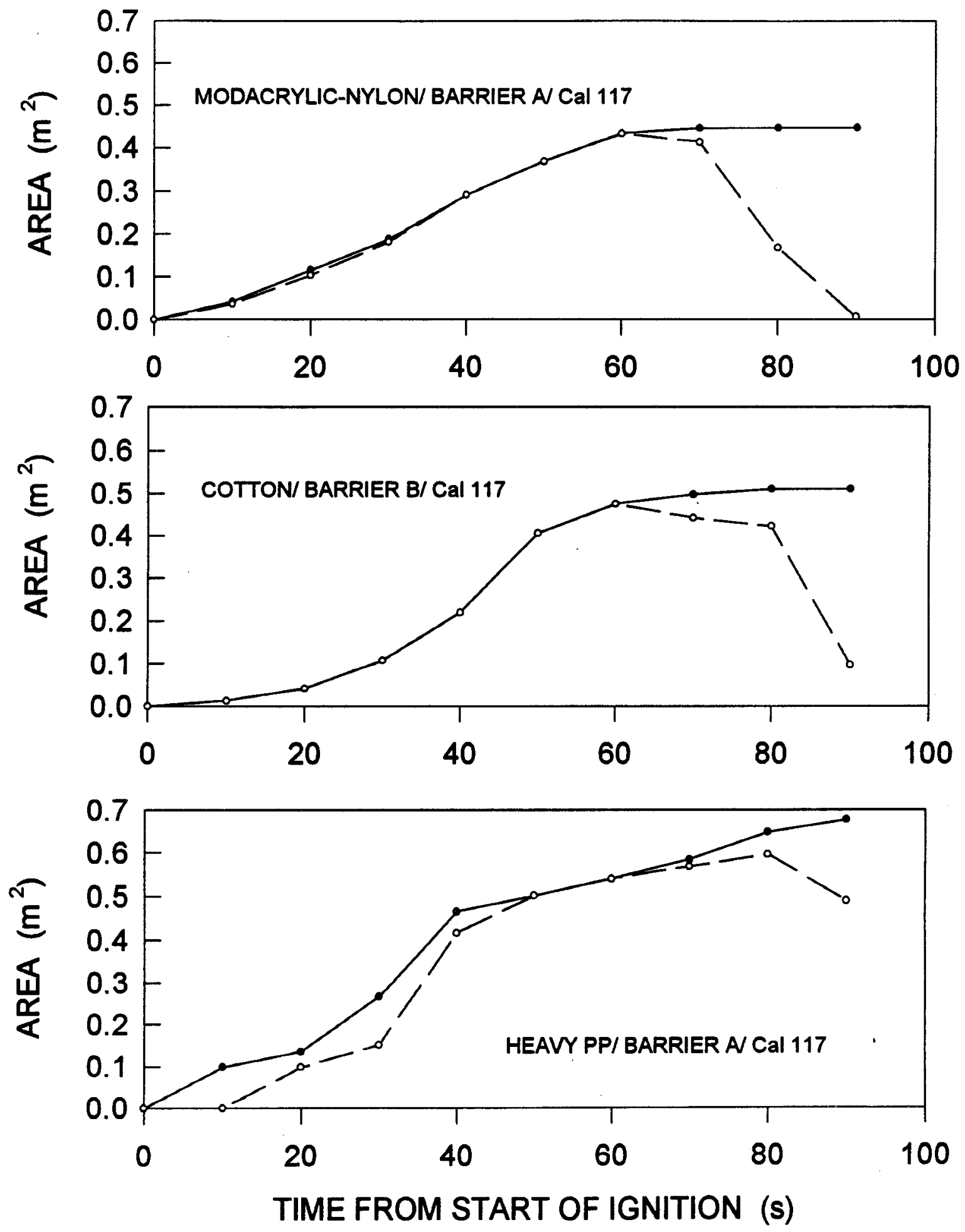
FIGURE 29

HEAT FLUXES NEAR

REAR CORNER OF SEAT

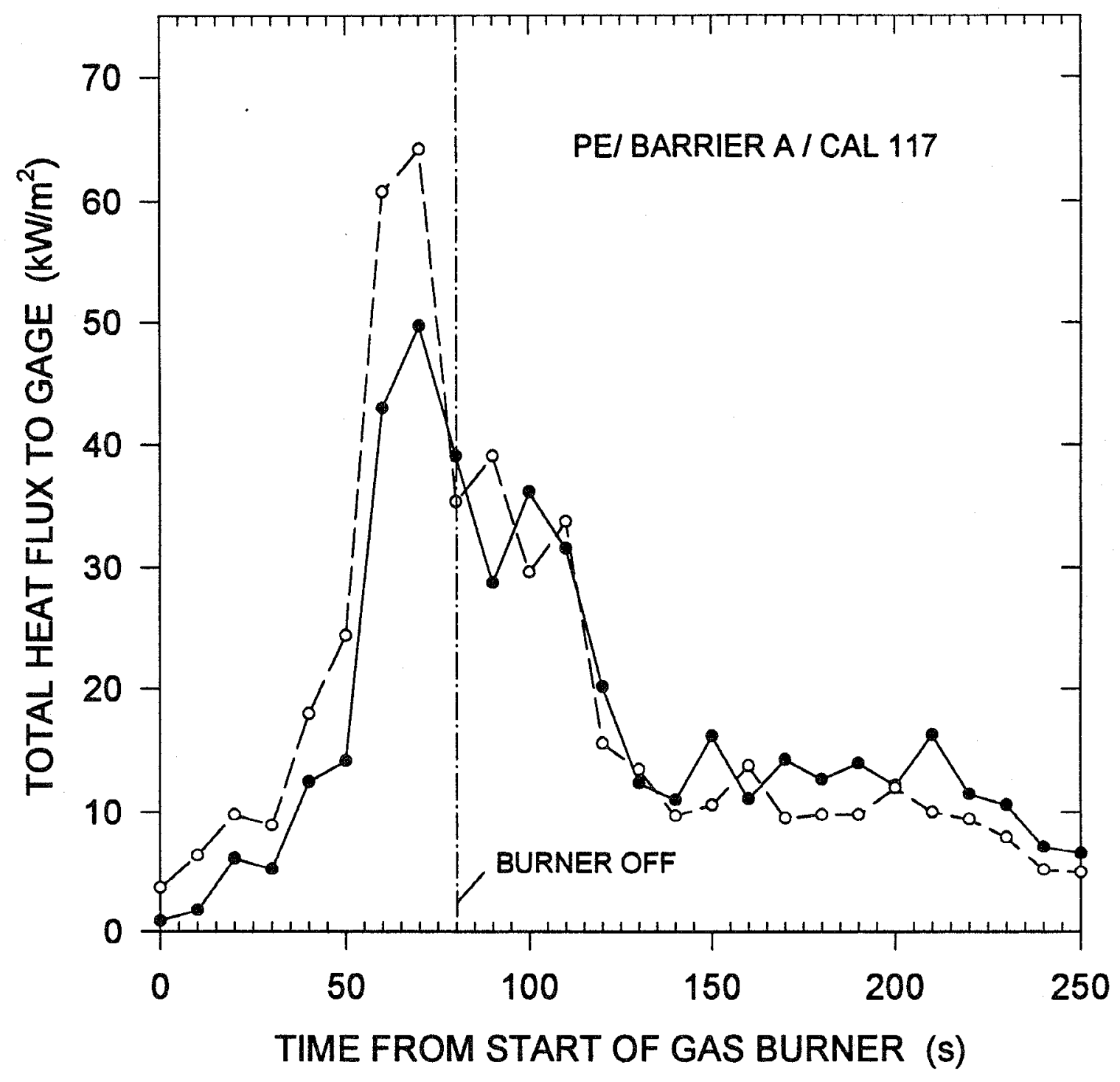


FIGURE 30

\section{CORRELATION BETWEEN HEAT FLUX AND HEAT RELEASE RATE DURING BURNER EXPOSURE}

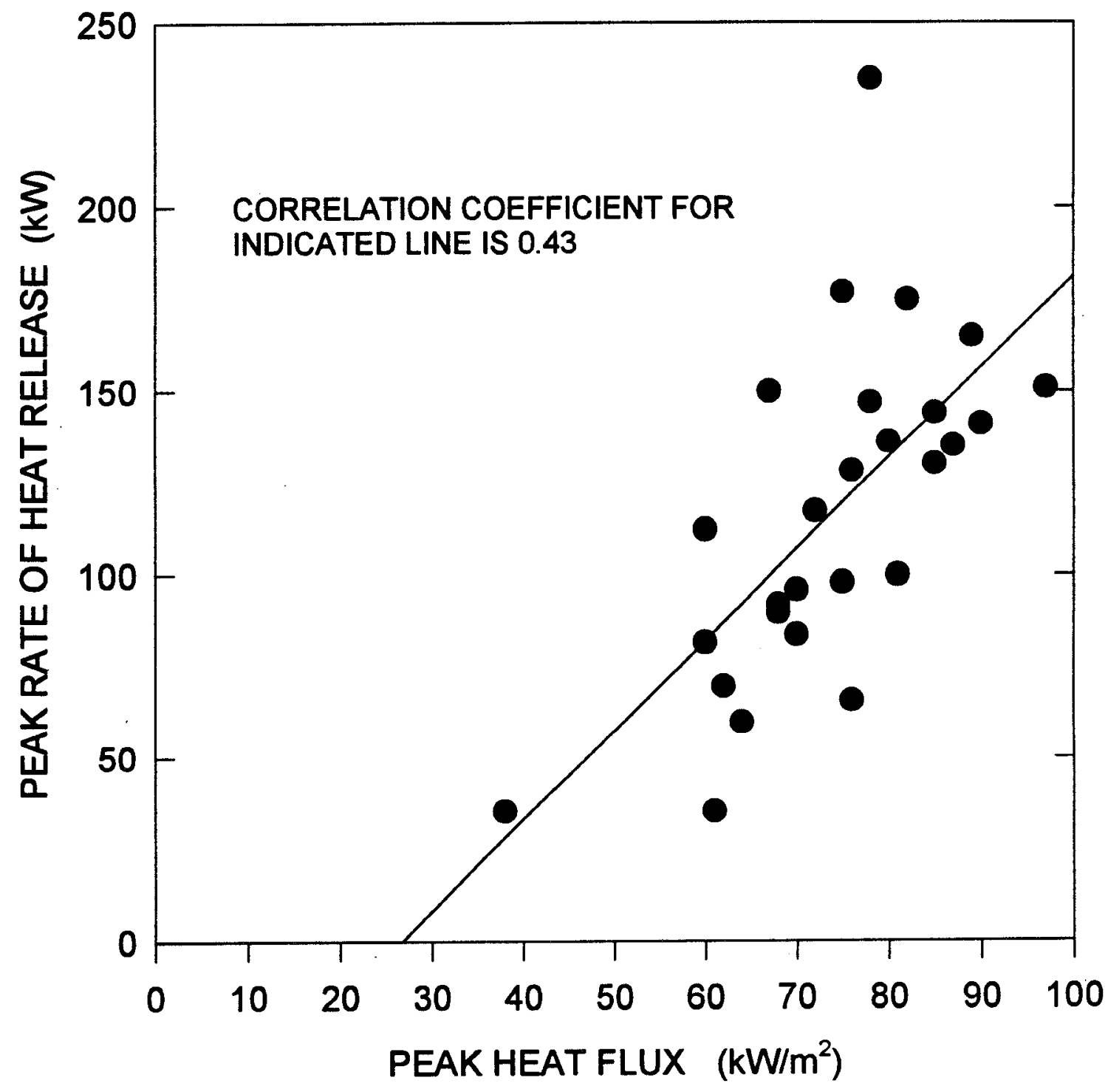


FIGURE 31 a

\section{Correlation Between 3 Minute Average Cone RHR And Highest Full Scale Peak RHR}

(See Figure $31 \mathrm{~b}$ for Legend)

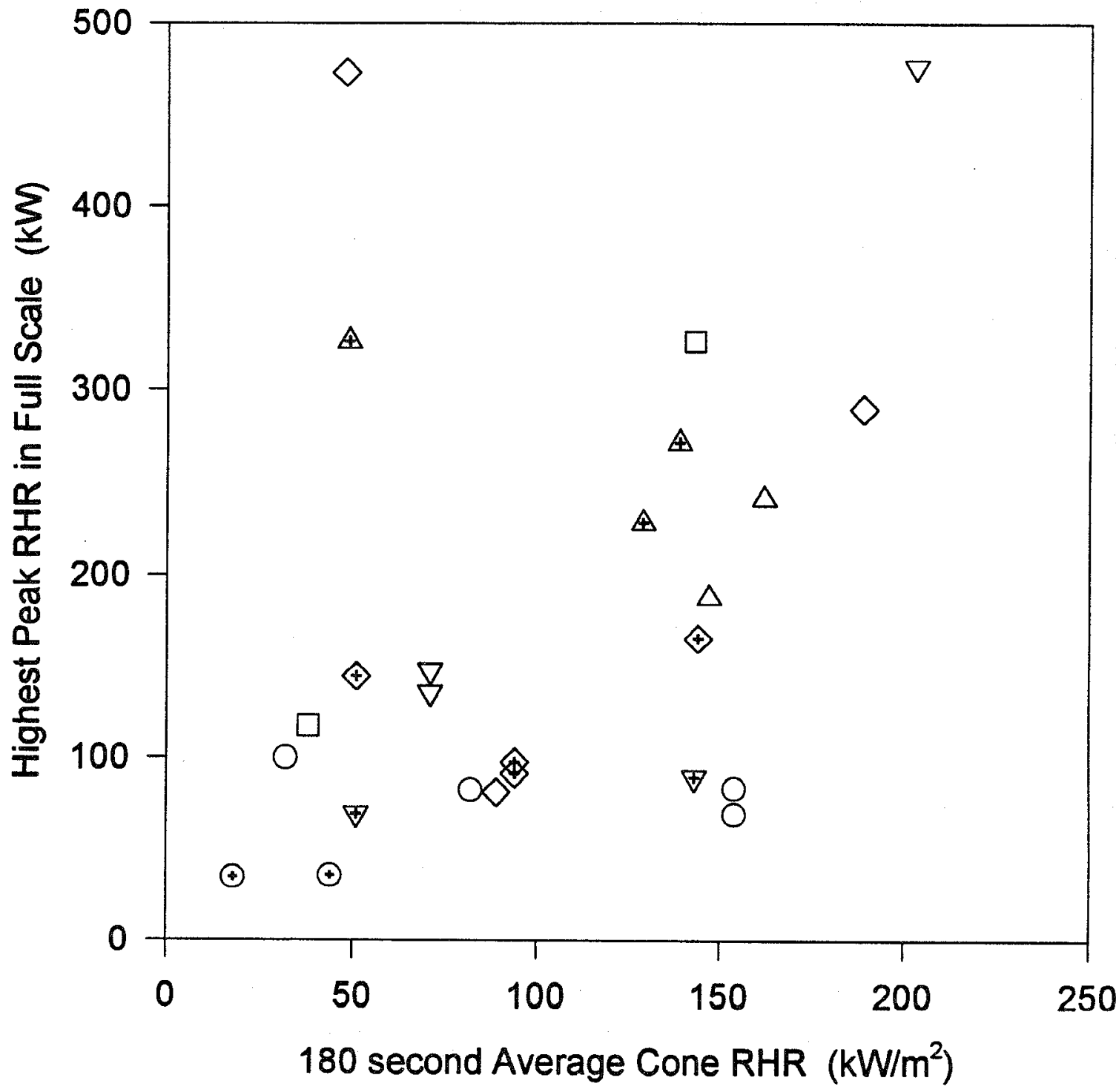


FIGURE $31 \mathrm{~b}$

LINEAR FIT BETWEEN CONE AVG RHR AND FIRST FULL-SCALE RHR PEAK

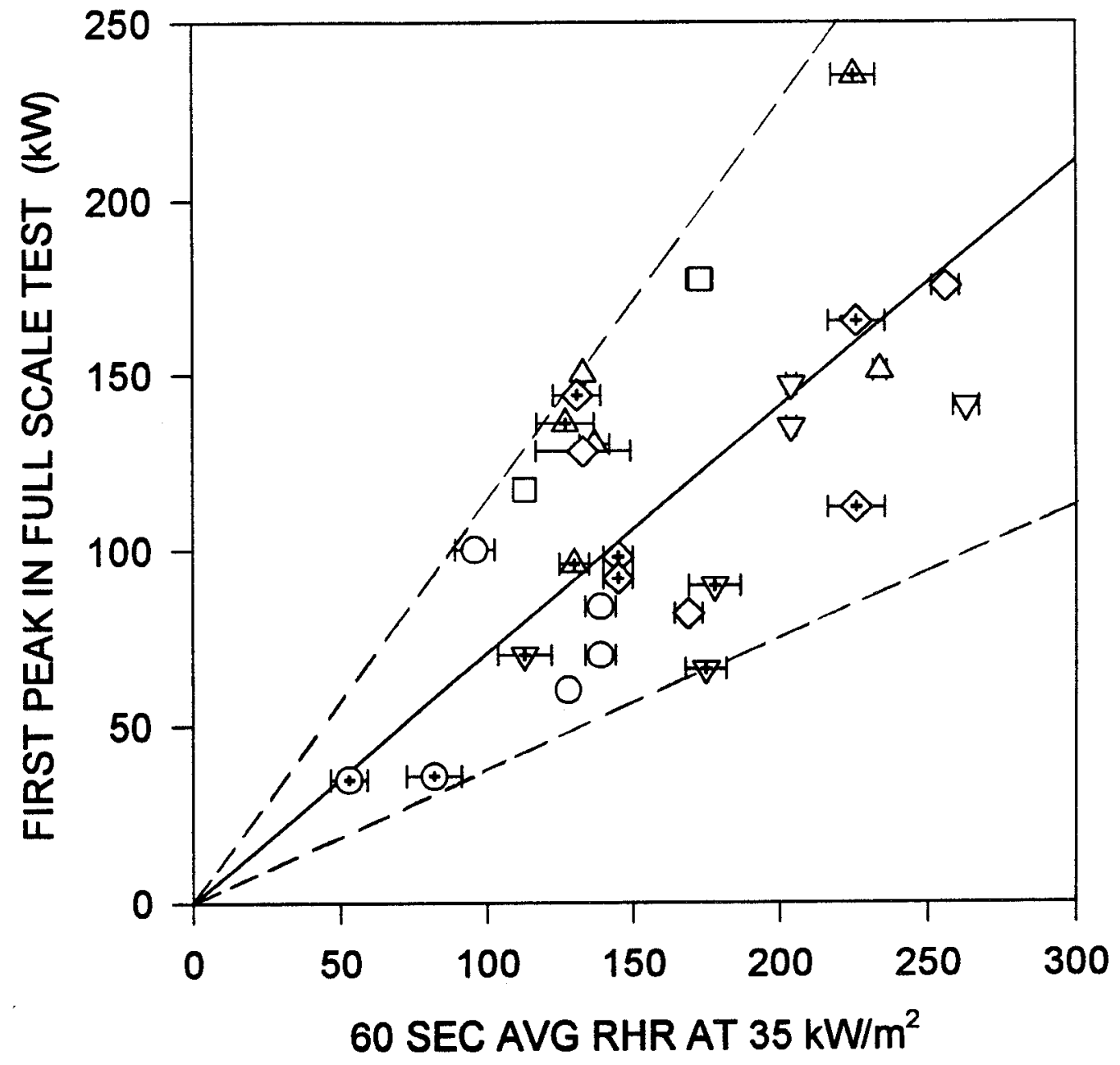

○ POLYESTER / CAL 117

$\nabla \quad$ NYLON / CAL 117

POLYESTER - COTTON / CAL 117

$\triangle \quad$ LIGHT PP / CAL 117

A LIGHT PP / IFR FOAM

$\diamond$ HEAVY PP / CAL 117

^ HEAVY PP / IFR FOAM

$\odot \quad$ MODACRYLIC - NYLON / CAL 117

$\nabla \quad$ COTTON / CAL 117 
Figure 32 a

\section{Correlation Based on Charring Fabrics Only (Cotton, Polyester/Cotton, Modacrylic/Nylon)}

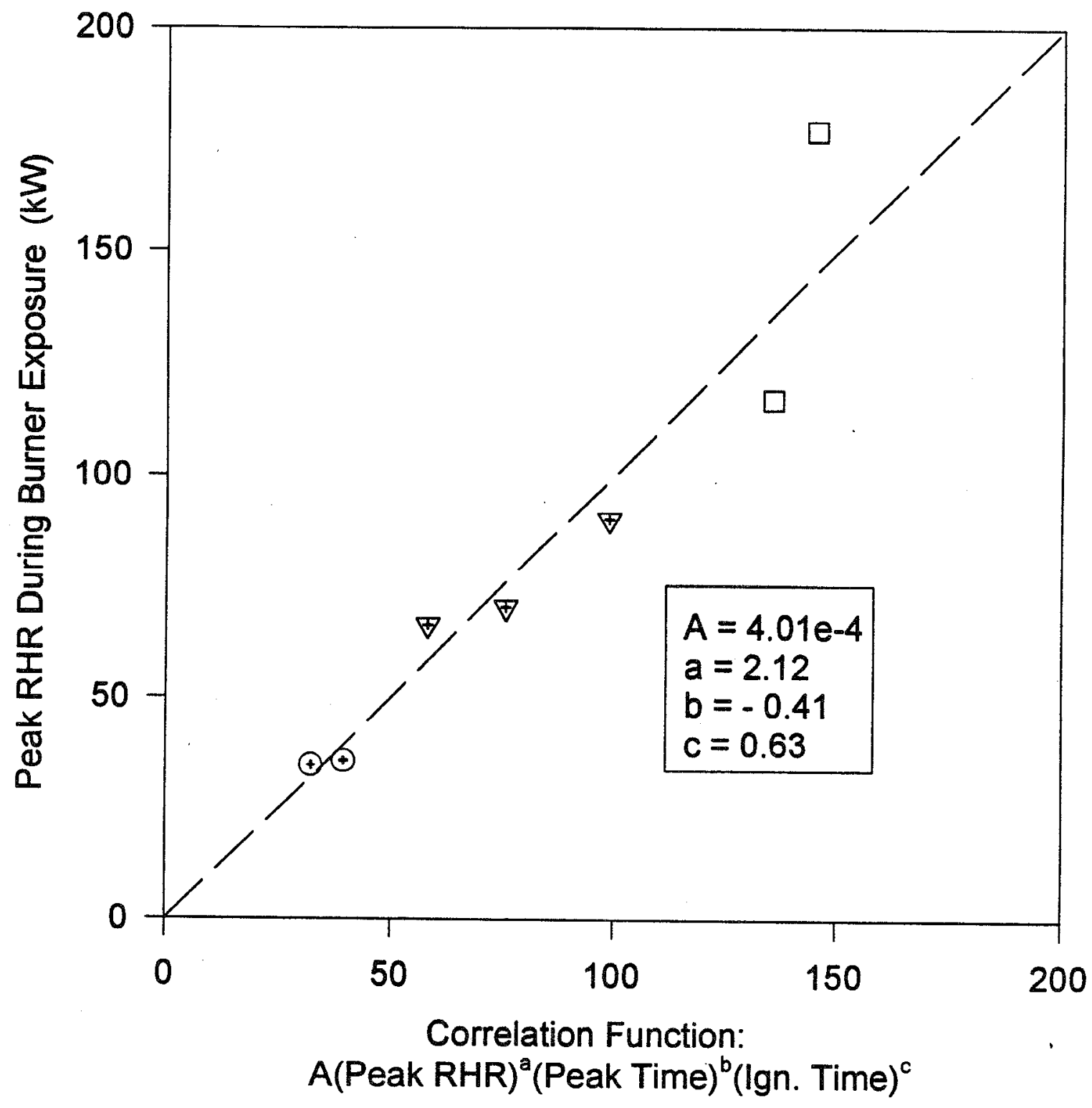


FIGURE $32 \mathrm{~b}$

CORRELATION OF FULL DATA SET

WITH POWER FUNCTION OF

CONE PEAK, CONE PEAK HALF HGT. TIME AND IGN. TIME

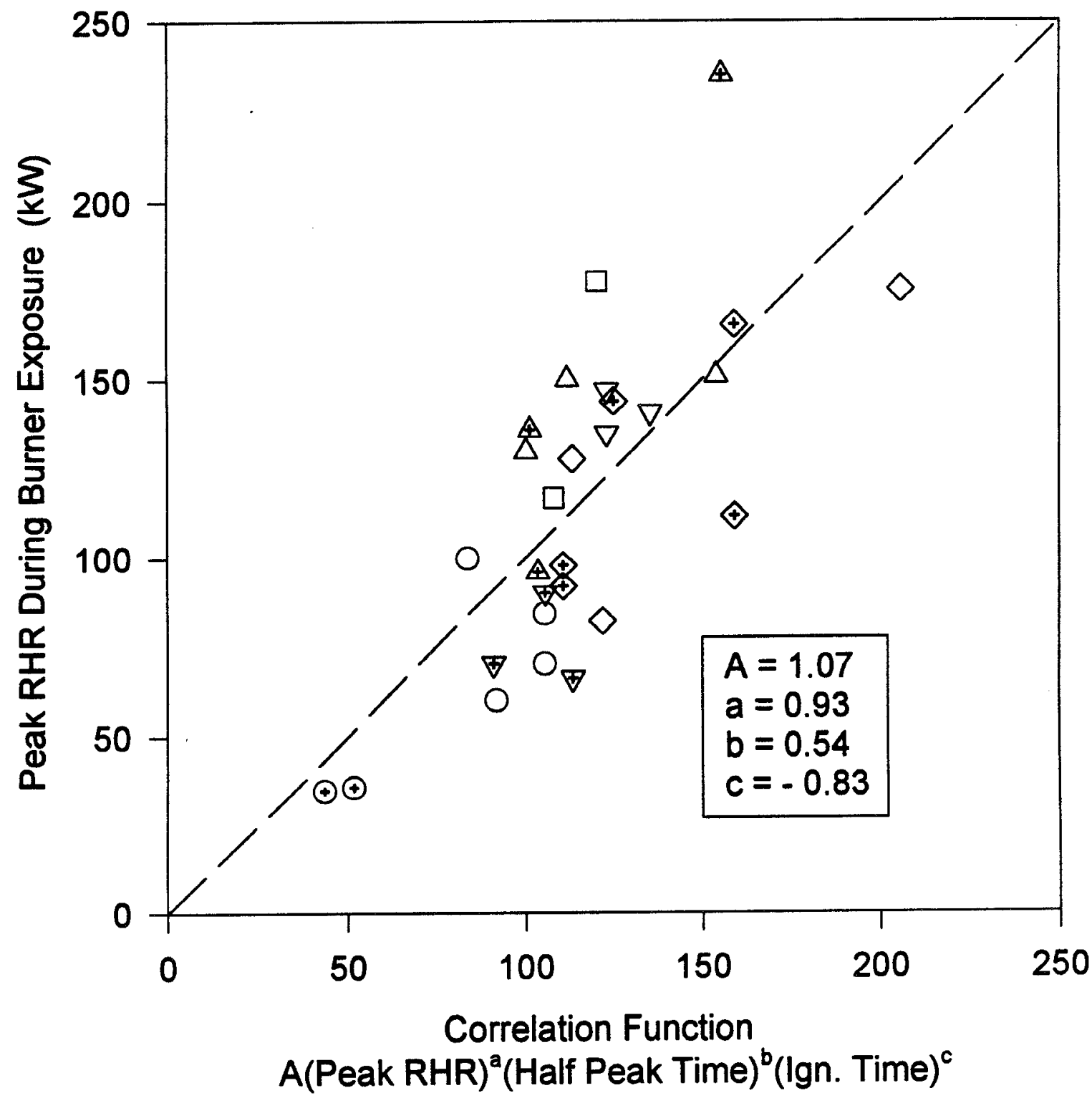


Figure $32 \mathrm{c}$

CORRELATION BASED ON

FOUR PARAMETERS FROM CONE CALORIMETER

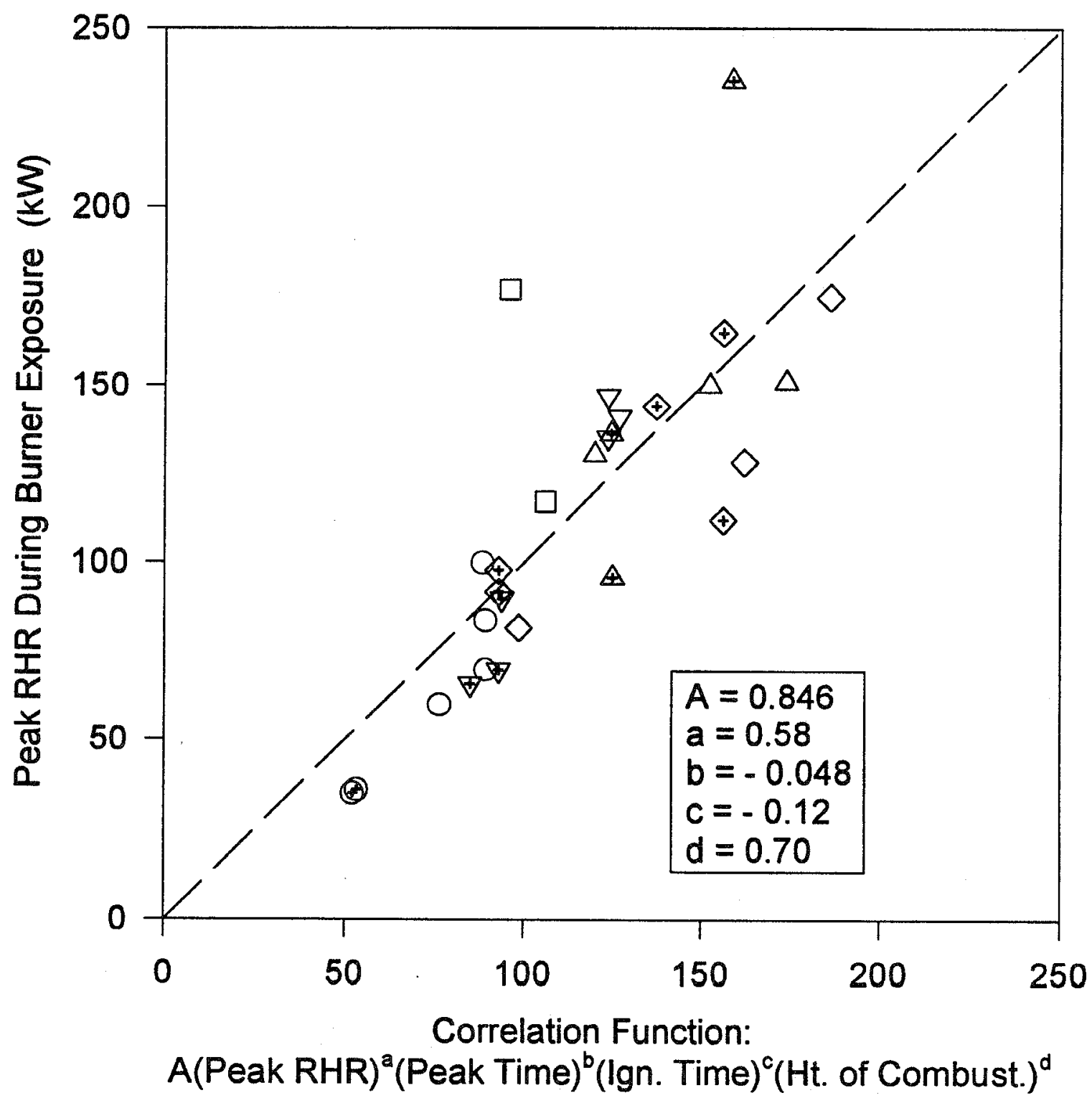


Figure 33 a

PEAK RATE OF HEAT RELEASE AFTER BURNER EXPOSURE

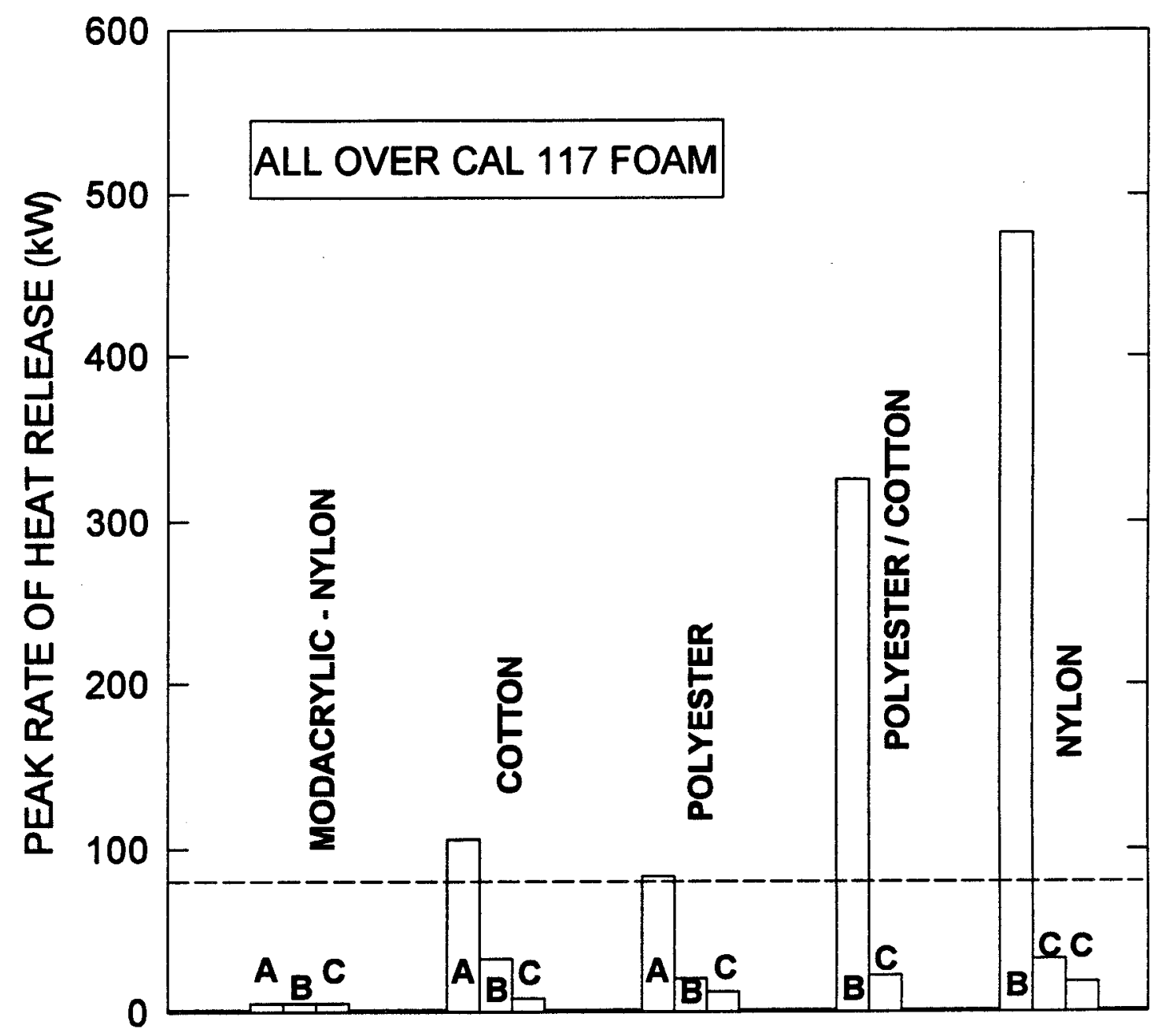

COVER FABRIC 
Figure $33 b$

PEAK RATE OF HEAT RELEASE AFTER BURNER EXPOSURE (100\% POLYPROPYLENE FABRICS)

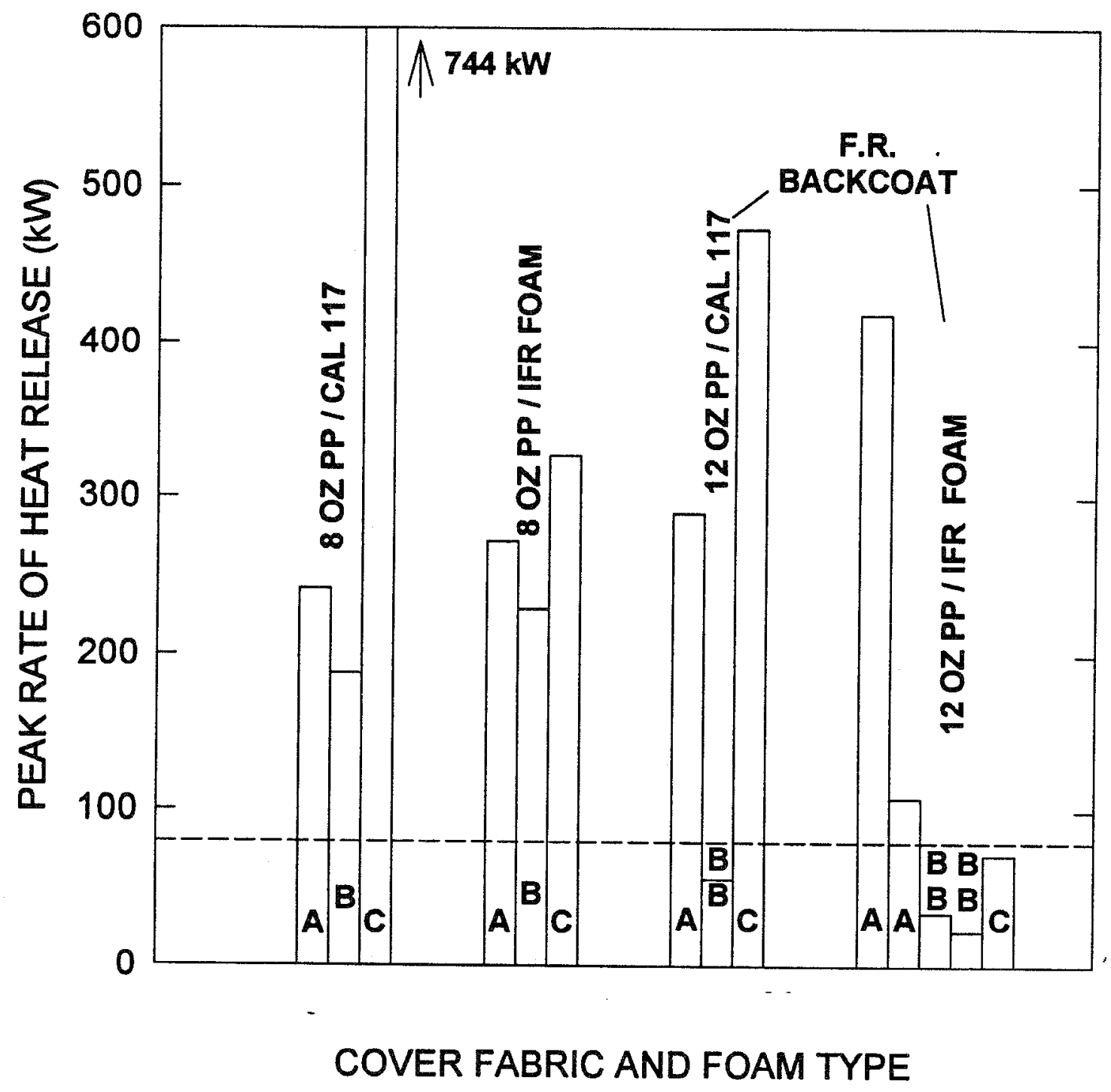




\section{"BASAL MELT FIRE " \\ ON CUSHIONS WITH BARRIERS}

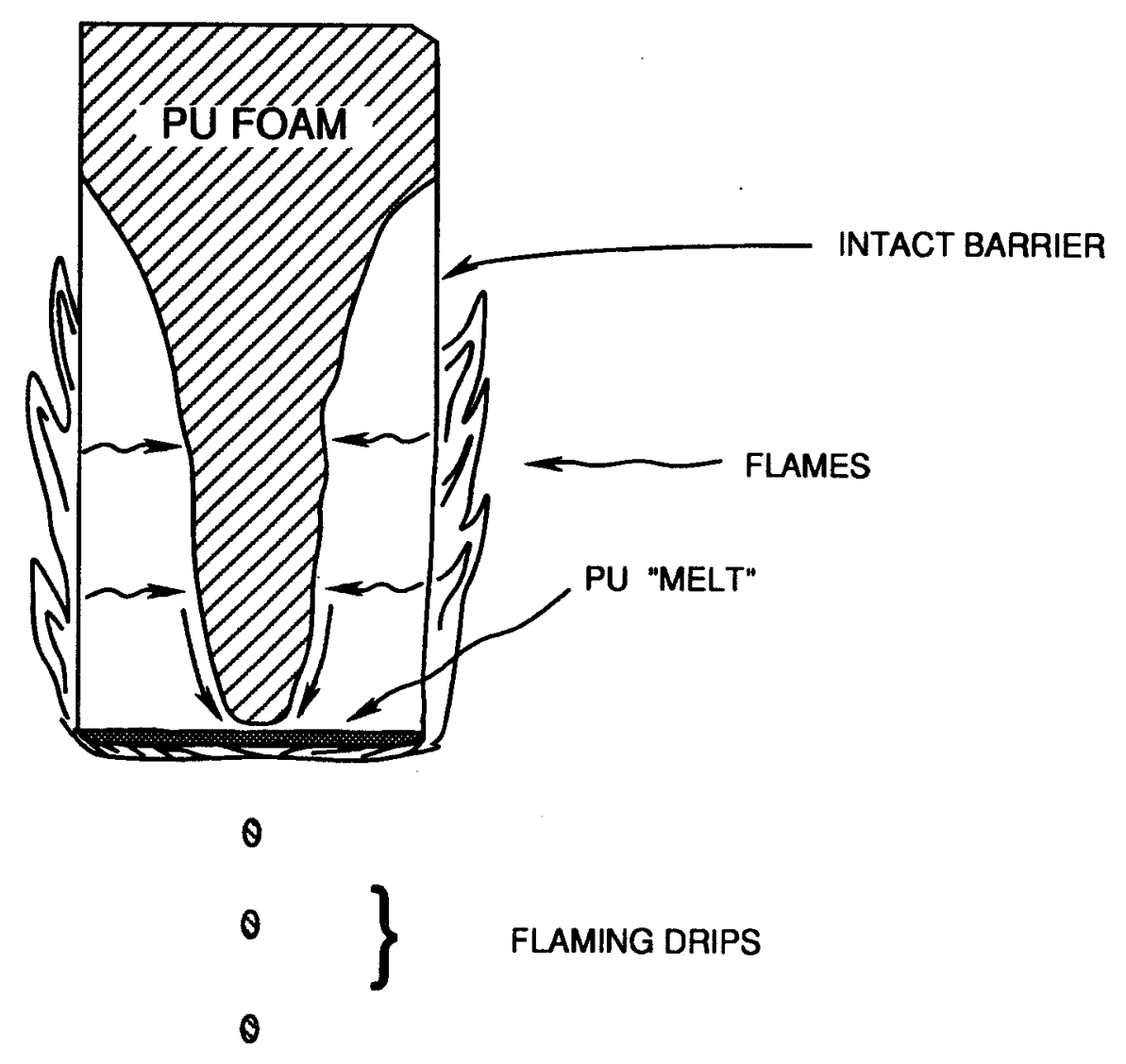

FIGURE 34 MECHANISM OF BARRIER FAILURE IN CB 133 MOCK - UP TESTS 
FIGURE 35

CORRELATION BETWEEN CONE AVG RHR AND SECOND FULL-SCALE PEAK RHR

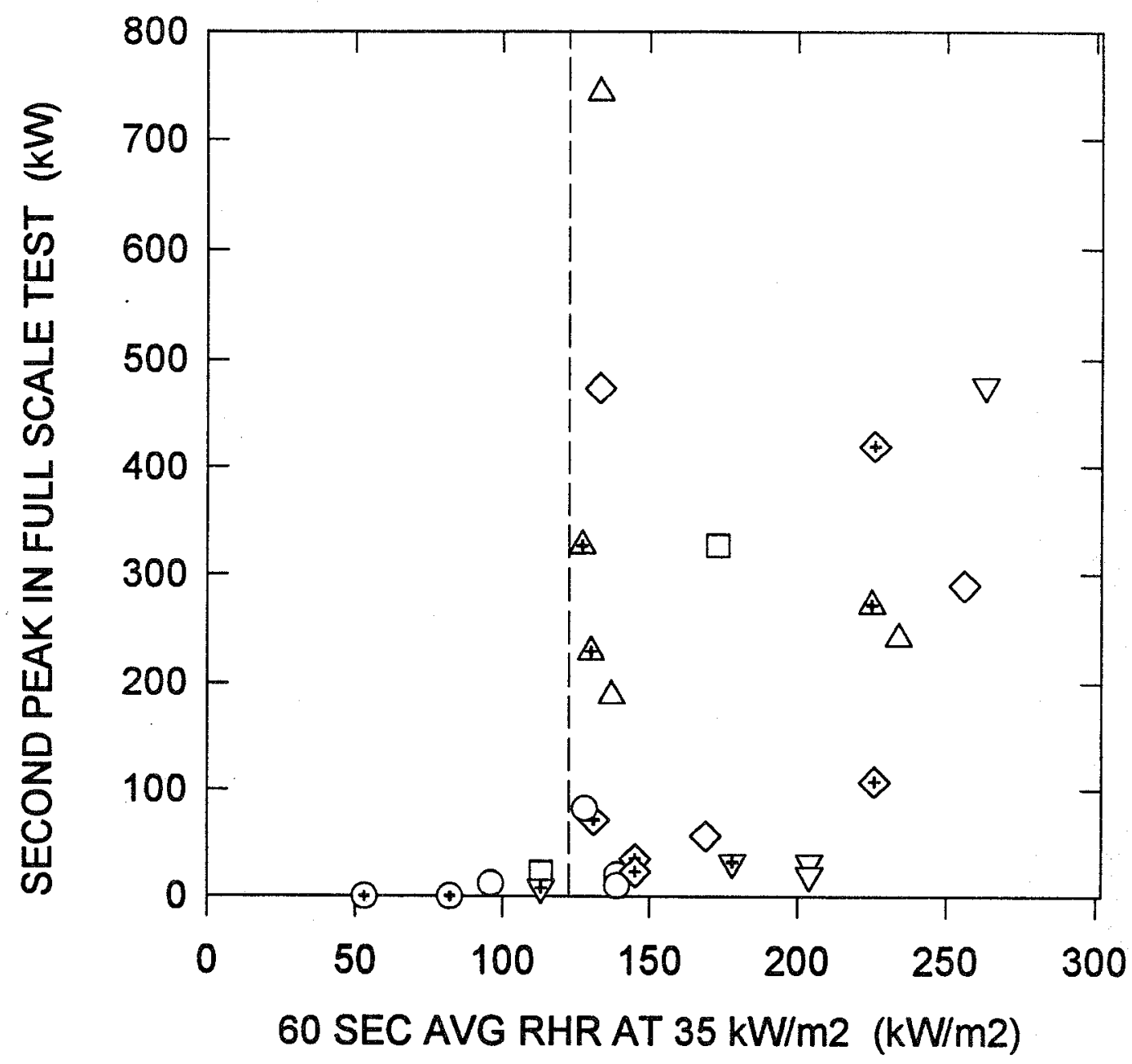

O POLYESTER / CAL 117

$\nabla \quad$ NYLON / CAL 117

$\square \quad$ POLYESTER - COTTON / CAL 117

$\triangle \quad$ LIGHT PP / CAL 117

A LIGHT PP / IFR FOAM

$\diamond$ HEAVY PP / CAL 117

$\odot$ HEAVY PP / IFR FOAM

๑) MODACRYLIC - NYLON / CAL 117

$\nabla$ COTTON / CAL 117 